\title{
ARITY SHAPE OF POLYADIC ALGEBRAIC STRUCTURES
}

\author{
STEVEN DUPLIJ
}

\begin{abstract}
Concrete two-set (module-like and algebra-like) algebraic structures are investigated from the viewpoint that the initial arities of all operations are arbitrary. Relations between operations arising from the structure definitions, however, lead to restrictions which determine their possible arity shapes and lead us to the partial arity freedom principle. In this manner, polyadic vector spaces and algebras, dual vector spaces, direct sums, tensor products and inner pairing spaces are reconsidered. As one application, elements of polyadic operator theory are outlined: multistars and polyadic analogs of adjoints, operator norms, isometries and projections are introduced, as well as polyadic $C^{*}$-algebras, Toeplitz algebras and Cuntz algebras represented by polyadic operators. Another application is connected with number theory, and it is shown that congruence classes are polyadic rings of a special kind. Polyadic numbers are introduced (see Definition 6.16), and Diophantine equations over these polyadic rings are then considered. Polyadic analogs of the Lander-Parkin-Selfridge conjecture and Fermat's last theorem are formulated. For nonderived polyadic ring operations (on polyadic numbers) neither of these statements holds, and counterexamples are given. Finally, a procedure for obtaining new solutions to the equal sums of like powers equation over polyadic rings by applying Frolov's theorem to the Tarry-Escott problem is presented.
\end{abstract}

\section{Contents}

INTRODUCTION

1. ONE SET POLYADIC “LINEAR” STRUCTURES

1.1. POLYADIC DISTRIBUTIVITY

1.2. POLYADIC RINGS AND FIELDS

2. TWO SET POLYADIC STRUCTURES

2.1. POLYADIC VECTOR SPACES

2.2. ONE-SET POLYADIC VECTOR SPACE

2.3. POLYADIC ALGEBRAS

3. MAPPINGS BETWEEN POLYADIC ALGEBRAIC STRUCTURES

3.1. POLYADIC FUNCTIONALS AND DUAL POLYADIC VECTOR SPACES

3.2. POLYADIC DIRECT SUM AND TENSOR PRODUCT

4. POLYADIC INNER PAIRING SPACES AND NORMS

APPLICATIONS

5. ELEMENTS OF POLYADIC OPERATOR THEORY

5.1. MULTISTARS AND POLYADIC ADJOINTS

5.2. POLYADIC ISOMETRY AND PROJECTION

5.3. TOWARDS POLYADIC ANALOG OF $C^{*}$-ALGEBRAS

6. CONGRUENCE CLASSES AS POLYADIC RINGS

6.1. POLYADIC RING ON INTEGERS

6.2. LIMITING CASES

7. EQUAL SUMS OF LIKE POWERS DIOPHANTINE EQUATION OVER POLYADIC INTEGERS

7.1. Polyadic ANAlog of THE LANDER-PARKIN-SElFridge CONJECTURE

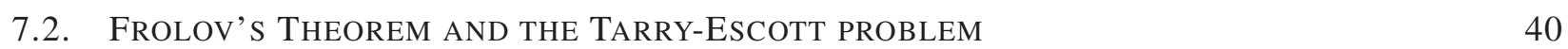

ACKNOWLEDGMENTS

REFERENCES

Date: March 29, 2017.

2010 Mathematics Subject Classification. 11D41, 11R04, 11R06, 17A42, 20N15, 47A05, 47L30, 47L70, 47L80. 


\section{INTRODUCTION}

The study of polyadic (higher arity) algebraic structures has a two-century long history, starting with works by Cayley, Sylvester, Kasner, Prüfer, Dörnte, Lehmer, Post, etc. They took a single set, closed under one (main) binary operation having special properties (the so called grouplike structure), and "generalized" it by increasing the arity of that operation, which can then be called a polyadic operation and the corresponding algebraic structure polyadic as well]. An "abstract way" to study polyadic algebraic structures is via the use of universal algebras defined as sets with different axioms (equational laws) for polyadic operations COHN [1965], GRÄTSER [1968], BERGMAN [2012]. However, in this language some important algebraic structures cannot be described, e.g. ordered groups, fields, etc. DENECKE AND WISMATH [2009]. Therefore, another "concrete approach" is to study examples of binary algebraic structures and then to "polyadize" them properly. This initiated the development of a corresponding theory of $n$-ary quasigroups BELOUSOV [1972], $n$-ary semigroups MONK AND SIOSON [1966], ZUPNIK [1967] and $n$-ary groups GAL'MAK [2003], RUSAKOV [1998] (for a more recent review, see, e.g., DUPLIJ [2012] and a comprehensive list of references therein). The binary algebraic structures with two operations (addition and multiplication) on one set (the so-called ring-like structures) were later on generalized to $(m, n)$-rings CELAKOSKI [1977], CROMBEZ [1972], LEESON AND BUTSON [1980] and $(m, n)$ fields IANCU AND POP [1997], while these were investigated mostly in a more restrictive manner by considering particular cases: ternary rings (or (2,3)-rings) LISTER [1971], ( $m, 2$ )-rings BOCCIONI [1965], POP AND POP [2002], as well as (3, 2)-fields DuPliJ AND WeRnER [2015].

In the case of one set, speaking informally, the "polyadization" of two operations" "interaction" is straightforward, giving only polyadic distributivity which does not connect or restrict their arities. However, when the number of sets becomes greater than one, the "polyadization" turns out to be nontrivial, leading to special relations between the operation arities, and also introduces additional (to arities) parameters, allowing us to classify them. We call a selection of such relations an arity shape and formulate the arity partial freedom principle that not all arities of the operations that arise during "polyadization" of binary operations are possible.

In this paper we consider two-set algebraic structures in the "concrete way" and provide the consequent "polyadization" of binary operations on them for the so-called module-like structures (vector spaces) and algebra-like structures (algebras and inner product spaces). The "polyadization" of binary scalar multiplication is defined in terms of the multiactions introduced in DUPLIJ [2012], having special arity shapes parametrized by the number of intact elements $\left(\ell_{\mathrm{id}}\right)$ in the corresponding multiactions. We then "polyadize" related constructions, such as dual vector spaces and direct sums, and also tensor products, and show that, as opposed to the binary case, they can be implemented in spaces of different arity signatures. The "polyadization" of inner product spaces and related norms gives additional arity shapes and restrictions. In the resulting TABLE 2 we present the arity signatures and shapes of the polyadic algebraic structures under consideration.

In the application part we note some starting points for polyadic operator theory by introducing multistars and polyadic analogs of adjoints, operator norms, isometries and projections. It is proved (Theorem 5.7) that, if the polyadic inner pairing (the analog of the inner product) is symmetric, then all multistars coincide and all polyadic operators are self-adjoint (in contrast to the binary case). The

\footnotetext{
${ }^{1}$ We use the term "polyadic" in this sense only, while there are other uses in the literature (see, e.g., HALMOS [1962]).
} 
polyadic analogs of $C^{*}$-algebras, Toeplitz algebras and Cuntz algebras are presented in terms of the polyadic operators introduced here, and a ternary example is given.

Another application is connected with number theory: we show that the internal structure of the congruence classes is described by a polyadic ring having a special arity signature (TABLE 3), and these we call polyadic integers (or numbers) $\mathbb{Z}_{(m, n)}$ (Definition 6.17). They are classified by polyadic shape invariants, and the relations between them which give the same arity signature are established. Also, the limiting cases are analyzed, and it is shown that in one such case the polyadic rings can be embedded into polyadic fields with binary multiplication, which leads to the so-called polyadic rational numbers CROMBEZ AND TIMM [1972]. We then consider Diophantine equations over these polyadic rings in a straightforward manner: we change only the arities of the operations ("additions" and "multiplications"), but save their mutual "interaction". In this way we try to "polyadize" the equal sums of like powers equation and formulate polyadic analogs of the Lander-Parkin-Selfridge conjecture and of Fermat's last theorem LANDER ET AL. [1967]. It is shown, that in the simplest case, when the polyadic "addition" and "multiplication" are nonderived (e.g., for polyadic numbers), neither conjecture is valid, and counterexamples are presented. Finally, we apply Frolov's theorem to the Tarry-Escott problem DORWART AND BROWN [1937], NGUYEN [2016] over polyadic rings to obtain new solutions to the equal sums of like powers equation for fixed congruence classes.

\section{ONE SET POLYADIC “LINEAR” STRUCTURES}

We use concise notations from our previous work on polyadic structures DUPLIJ [2012, 2016]. Take a non-empty set $A$, then $n$-tuple (or polyad) consisting of the elements $\left(a_{1}, \ldots, a_{n}\right), a_{i} \in A$, is denoted by bold letter $(\boldsymbol{a})$ taking it values in the Cartesian product $A^{\times n}$. If the number of elements in the $n$-tuple is important, we denote it $\left(\boldsymbol{a}^{(n)}\right)$, and an $n$-tuple with equal elements is denoted by $\left(a^{n}\right)$. On the Cartesian product $A^{\times n}$ one can define a polyadic operation $\boldsymbol{\mu}_{n}: A^{\times n} \rightarrow A$, and use the notation $\boldsymbol{\mu}_{n}[\boldsymbol{a}]$. A polyadic structure $\mathcal{A}$ is a set $A$ which is closed under polyadic operations, and a polyadic signature is the selection of their arities. For formal definitions, see, e.g., COHN [1965].

1.1. Polyadic distributivity. Let us consider a polyadic structure with two operations on the same set $A$ : the "chief" (multiplication) $n$-ary operation $\boldsymbol{\mu}_{n}: A^{n} \rightarrow A$ and the additional $m$-ary operation $\boldsymbol{\nu}_{m}: A^{m} \rightarrow A$, that is $\left\langle A \mid \boldsymbol{\mu}_{n}, \boldsymbol{\nu}_{m}\right\rangle$. If there are no relations between $\boldsymbol{\mu}_{n}$ and $\boldsymbol{\nu}_{m}$, then nothing new, as compared with the polyadic structures having a single operation $\left\langle A \mid \boldsymbol{\mu}_{n}\right\rangle$ or $\left\langle A \mid \boldsymbol{\nu}_{m}\right\rangle$, can be said. Informally, the "interaction" between operations can be described using the important relation of distributivity (an analog of $a \cdot(b+c)=a \cdot b+a \cdot c, a, b, c \in A$ in the binary case).

Definition 1.1. The polyadic distributivity for the operations $\boldsymbol{\mu}_{n}$ and $\boldsymbol{\nu}_{m}$ (no additional properties are implied for now) consists of $n$ relations

$$
\begin{aligned}
& \boldsymbol{\mu}_{n}\left[\boldsymbol{\nu}_{m}\left[a_{1}, \ldots a_{m}\right], b_{2}, b_{3}, \ldots b_{n}\right] \\
& =\boldsymbol{\nu}_{m}\left[\boldsymbol{\mu}_{n}\left[a_{1}, b_{2}, b_{3}, \ldots b_{n}\right], \boldsymbol{\mu}_{n}\left[a_{2}, b_{2}, b_{3}, \ldots b_{n}\right], \ldots \boldsymbol{\mu}_{n}\left[a_{m}, b_{2}, b_{3}, \ldots b_{n}\right]\right] \\
& \boldsymbol{\mu}_{n}\left[b_{1}, \boldsymbol{\nu}_{m}\left[a_{1}, \ldots a_{m}\right], b_{3}, \ldots b_{n}\right] \\
& =\boldsymbol{\nu}_{m}\left[\boldsymbol{\mu}_{n}\left[b_{1}, a_{1}, b_{3}, \ldots b_{n}\right], \boldsymbol{\mu}_{n}\left[b_{1}, a_{2}, b_{3}, \ldots b_{n}\right], \ldots \boldsymbol{\mu}_{n}\left[b_{1}, a_{m}, b_{3}, \ldots b_{n}\right]\right] \\
& \vdots \\
& \boldsymbol{\mu}_{n}\left[b_{1}, b_{2}, \ldots b_{n-1}, \boldsymbol{\nu}_{m}\left[a_{1}, \ldots a_{m}\right]\right] \\
& =\boldsymbol{\nu}_{m}\left[\boldsymbol{\mu}_{n}\left[b_{1}, b_{2}, \ldots b_{n-1}, a_{1}\right], \boldsymbol{\mu}_{n}\left[b_{1}, b_{2}, \ldots b_{n-1}, a_{2}\right], \ldots \boldsymbol{\mu}_{n}\left[b_{1}, b_{2}, \ldots b_{n-1}, a_{m}\right]\right]
\end{aligned}
$$

where $a_{i}, b_{j} \in A$.

It is seen that the operations $\boldsymbol{\mu}_{n}$ and $\boldsymbol{\nu}_{m}$ enter into (1.1)-(1.3) in a non-symmetric way, which allows us to distinguish them: one of them ( $\boldsymbol{\mu}_{n}$, the $n$-ary multiplication) "distributes" over the other 
one $\boldsymbol{\nu}_{m}$, and therefore $\boldsymbol{\nu}_{m}$ is called the addition. If only some of the relations (1.1)-(1.3) hold, then such distributivity is partial (the analog of left and right distributivity in the binary case). Obviously, the operations $\boldsymbol{\mu}_{n}$ and $\boldsymbol{\nu}_{m}$ need have nothing to do with ordinary multiplication (in the binary case denoted by $\boldsymbol{\mu}_{2} \Longrightarrow(\cdot)$ ) and addition (in the binary case denoted by $\nu_{2} \Longrightarrow(+)$ ), as in the example below.

Example 1.2. Let $A=\mathbb{R}, n=2, m=3$, and $\boldsymbol{\mu}_{2}\left[b_{1}, b_{2}\right]=b_{1}^{b_{2}}, \boldsymbol{\nu}_{3}\left[a_{1}, a_{2}, a_{3}\right]=a_{1} a_{2} a_{3}$ (product in $\mathbb{R}$ ). The partial distributivity now is $\left(a_{1} a_{2} a_{3}\right)^{b_{2}}=a_{1}^{b_{2}} a_{2}^{b_{2}} a_{3}^{b_{2}}$ (only the first relation (1.1) holds).

1.2. Polyadic rings and fields. Here we briefly remind the reader of one-set (ring-like) polyadic structures (informally). Let both operations $\boldsymbol{\mu}_{n}$ and $\boldsymbol{\nu}_{m}$ be (totally) associative, which (in our definition DUPLIJ [2012]) means independence of the composition of two operations under placement of the internal operations (there are $n$ and $m$ such placements and therefore $(n+m)$ corresponding relations)

$$
\begin{aligned}
\boldsymbol{\mu}_{n}\left[\boldsymbol{a}, \boldsymbol{\mu}_{n}[\boldsymbol{b}], \boldsymbol{c}\right] & =\text { invariant } \\
\boldsymbol{\nu}_{m}\left[\boldsymbol{d}, \boldsymbol{\nu}_{m}[\boldsymbol{e}], \boldsymbol{f}\right] & =\text { invariant }
\end{aligned}
$$

where the polyads $\boldsymbol{a}, \boldsymbol{b}, \boldsymbol{c}, \boldsymbol{d}, \boldsymbol{e}, \boldsymbol{f}$ have corresponding length, and then both $\left\langle A\left|\boldsymbol{\mu}_{n}\right| a s s o c\right\rangle$ and $\left\langle A\left|\boldsymbol{\nu}_{m}\right|\right.$ assoc $\rangle$ are polyadic semigroups $\mathcal{S}_{n}$ and $\mathcal{S}_{m}$. A commutative semigroup $\left\langle A\left|\boldsymbol{\nu}_{m}\right|\right.$ assoc,comm $\rangle$ is defined by $\nu_{m}[\boldsymbol{a}]=\nu_{m}[\sigma \circ \boldsymbol{a}]$, for all $\sigma \in S_{n}$, where $S_{n}$ is the symmetry group. If the equation $\nu_{m}[\boldsymbol{a}, x, \boldsymbol{b}]=c$ is solvable for any place of $x$, then $\left\langle A\left|\boldsymbol{\nu}_{m}\right| a s s o c\right.$, solv $\rangle$ is a polyadic group $\mathcal{G}_{m}$, and such $x=\tilde{c}$ is called a (additive) querelement for $c$, which defines the (additive) unary queroperation $\tilde{\nu}_{1}$ by $\tilde{\nu}_{1}[c]=\tilde{c}$.

Definition 1.3. A polyadic $(m, n)$-ring $\mathcal{R}_{m, n}$ is a set $A$ with two operations $\boldsymbol{\mu}_{n}: A^{n} \rightarrow A$ and $\boldsymbol{\nu}_{m}: A^{m} \rightarrow A$, such that: 1) they are distributive (1.1)-(1.3); 2) $\left\langle A\left|\mu_{n}\right|\right.$ assoc $\rangle$ is a polyadic semigroup; 3) $\left\langle A\left|\nu_{m}\right|\right.$ assoc, comm, solv $\rangle$ is a commutative polyadic group.

It is obvious that a $(2,2)$-ring $\mathcal{R}_{2,2}$ is an ordinary (binary) ring. Polyadic rings have much richer structure and can have unusual properties CELAKOSKI [1977], CROMBEZ [1972], ČUPONA [1965], LEESON AND BUTSON [1980]. If the multiplicative semigroup $\left\langle A\left|\boldsymbol{\mu}_{n}\right|\right.$ assoc $\rangle$ is commutative, $\boldsymbol{\mu}_{n}[\boldsymbol{a}]=\boldsymbol{\mu}_{n}[\sigma \circ \boldsymbol{a}]$, for all $\sigma \in S_{n}$, then $\mathcal{R}_{m, n}$ is called a commutative polyadic ring, and if it contains the identity, then $\mathcal{R}_{m, n}$ is a (polyadic) $(m, n)$-semiring. If the distributivity is only partial, then $\mathcal{R}_{m, n}$ is called a polyadic near-ring.

Introduce in $\mathcal{R}_{m, n}$ additive and multiplicative idempotent elements by $\boldsymbol{\nu}_{m}\left[a^{m}\right]=a$ and $\boldsymbol{\mu}_{n}\left[b^{n}\right]=b$, respectively. A zero $z$ of $\mathcal{R}_{m, n}$ is defined by $\boldsymbol{\mu}_{n}[z, \boldsymbol{a}]=z$ for any $\boldsymbol{a} \in A^{n-1}$, where $z$ can be on any place. Evidently, a zero (if it exists) is a multiplicative idempotent and is unique, and, if a polyadic ring has an additive idempotent, it is a zero LEESON AND BUTSON [1980]. Due to the distributivity (1.1)-(1.3), there can be at most one zero in a polyadic ring. If a zero $z$ exists, denote $A^{*}=A \backslash\{z\}$, and observe that (in distinction to binary rings) $\left\langle A^{*}\left|\boldsymbol{\mu}_{n}\right|\right.$ assoc $\rangle$ is not a polyadic group, in general. In the case where $\left\langle A^{*}\left|\boldsymbol{\mu}_{n}\right|\right.$ assoc $\rangle$ is a commutative $n$-ary group, such a polyadic ring is called a (polyadic) $(m, n)$-field and $\mathbb{K}_{m, n}$ ("polyadic scalars") (see LEESON AND BUTSON [1980], IANCU AND POP [1997]).

A multiplicative identity $e$ in $\mathcal{R}_{m, n}$ is a distinguished element $e$ such that

$$
\mu_{n}\left[a,\left(e^{n-1}\right)\right]=a,
$$

for any $a \in A$ and where $a$ can be on any place. In binary rings the identity is the only neutral element, while in polyadic rings there can exist many neutral $(n-1)$-polyads $e$ satisfying

$$
\mu_{n}[a, \boldsymbol{e}]=a,
$$


for any $a \in A$ which can also be on any place. The neutral polyads $e$ are not determined uniquely. Obviously, the polyad $\left(e^{n-1}\right)$ is neutral. There exist exotic polyadic rings which have no zero, no identity, and no additive idempotents at all (see, e.g., CROMBEZ [1972]), but, if $m=2$, then a zero always exists LEESON AND BUTSON [1980].

Example 1.4. Let us consider a polyadic ring $\mathcal{R}_{3,4}$ generated by 2 elements $a, b$ and the relations

$$
\begin{array}{ll}
\mu_{4}\left[a^{4}\right]=a, \quad \mu_{4}\left[a^{3}, b\right]=b, & \mu_{4}\left[a^{2}, b^{2}\right]=a, \quad \mu_{4}\left[a, b^{3}\right]=b, \quad \mu_{4}\left[b^{4}\right]=a, \\
\nu_{3}\left[a^{3}\right]=b, \quad \nu_{3}\left[a^{2}, b\right]=a, \quad \nu_{3}\left[a, b^{2}\right]=b, \quad \nu_{3}\left[b^{3}\right]=a,
\end{array}
$$

which has a multiplicative idempotent $a$ only, but has no zero and no identity.

Proposition 1.5. In the case of polyadic structures with two operations on one set there are no conditions between arities of operations which could follow from distributivity (1.1)-(1.3) or the other relations above, and therefore they have no arity shape.

Such conditions will appear below, when we consider more complicated universal algebraic structures with two or more sets with operations and relations.

\section{TWO SET POLYADIC STRUCTURES}

2.1. Polyadic vector spaces. Let us consider a polyadic field $\mathbb{K}_{m_{K}, n_{K}}=\left\langle K \mid \boldsymbol{\sigma}_{m_{K}}, \boldsymbol{\kappa}_{n_{K}}\right\rangle$ ("polyadic scalars"), having $m_{K}$-ary addition $\boldsymbol{\sigma}_{m_{K}}: K^{m_{K}} \rightarrow K$ and $n_{K}$-ary multiplication $\boldsymbol{\kappa}_{n_{K}}: K^{n_{K}} \rightarrow K$, and the identity $e_{K} \in K$, a neutral element with respect to multiplication $\boldsymbol{\kappa}_{n_{K}}\left[e_{K}^{n_{K}-1}, \lambda\right]=\lambda$, for all $\lambda \in K$. In polyadic structures, one can introduce a neutral $\left(n_{K}-1\right)$-polyad (identity polyad for "scalars") $e_{K} \in K^{n_{K}-1}$ by

$$
\boldsymbol{\kappa}_{n_{K}}\left[\boldsymbol{e}_{K}, \lambda\right]=\lambda
$$

where $\lambda \in K$ can be on any place.

Next, take a $m_{V}$-ary commutative (abelian) group $\left\langle\mathrm{V} \mid \boldsymbol{\nu}_{m_{V}}\right\rangle$, which can be treated as "polyadic vectors" with $m_{V}$-ary addition $\boldsymbol{\nu}_{m_{V}}: \mathrm{V}^{m_{V}} \rightarrow \mathrm{V}$. Define in $\left\langle\mathrm{V} \mid \boldsymbol{\nu}_{m_{V}}\right\rangle$ an additive neutral element (zero) $\mathrm{z}_{V} \in \mathrm{V}$ by

$$
\boldsymbol{\nu}_{m_{V}}\left[\mathrm{z}_{V}^{m_{V}-1}, \mathrm{v}\right]=\mathrm{v}
$$

for any $v \in \mathrm{V}$, and a "negative vector" $\bar{v} \in \mathrm{V}$ as its querelement

$$
\boldsymbol{\nu}_{m_{V}}\left[\boldsymbol{a}_{V}, \overline{\mathrm{v}}, \boldsymbol{b}_{V}\right]=\mathrm{v},
$$

where $\bar{v}$ can be on any place in the 1.h.s., and $a_{V}, b_{V}$ are polyads in V. Here, instead of one neutral element we can also introduce the $\left(m_{V}-1\right)$-polyad $z_{V}$ (which may not be unique), and so, for a zero polyad (for "vectors") we have

$$
\boldsymbol{\nu}_{m_{V}}\left[\mathrm{z}_{V}, \mathrm{v}\right]=\mathrm{v}, \quad \forall \mathrm{v} \in \mathrm{V},
$$

where $\vee \in V$ can be on any place. The "interaction" between "polyadic scalars" and "polyadic vectors" (the analog of binary multiplication by a scalar $\lambda v)$ can be defined as a multiaction $\left(k_{\rho}\right.$-place action) introduced in DUPLIJ [2012]

$$
\boldsymbol{\rho}_{k_{\rho}}: K^{k_{\rho}} \times \mathrm{V} \longrightarrow \mathrm{V}
$$

The set of all multiactions form a $n_{\rho}$-ary semigroup $\mathcal{S}_{\rho}$ under composition. We can "normalize" the multiactions in a similar way, as multiplace representations DUPLIJ [2012], by (an analog of $1 \mathrm{v}=\mathrm{v}$, $\mathrm{v} \in \mathrm{V}, 1 \in K$ )

$$
\boldsymbol{\rho}_{k_{\rho}}\left\{\begin{array}{c|c}
e_{K} & \\
\vdots & \mathrm{v} \\
e_{K} &
\end{array}\right\}=\mathrm{v}
$$


for all $\mathrm{v} \in \mathrm{V}$, where $e_{K}$ is the identity of $\mathbb{K}_{m_{K}, n_{K}}$. In the case of an (ordinary) 1-place (left) action (as an external binary operation) $\rho_{1}: K \times \mathrm{V} \rightarrow \mathrm{V}$, its consistency with the polyadic field multiplication $\boldsymbol{\kappa}_{n_{K}}$ under composition of the binary operations $\boldsymbol{\rho}_{1}\{\lambda \mid a\}$ gives a product of the same arity

$$
n_{\rho}=n_{K},
$$

that is (a polyadic analog of $\lambda(\mu \mathrm{v})=(\lambda \mu) \mathrm{v}, \mathrm{v} \in \mathrm{V}, \lambda, \mu \in K$ )

$$
\boldsymbol{\rho}_{1}\left\{\lambda_{1} \mid \boldsymbol{\rho}_{1}\left\{\lambda_{2}|\ldots| \boldsymbol{\rho}_{1}\left\{\lambda_{n_{K}} \mid \mathrm{v}\right\}\right\} \ldots\right\}=\boldsymbol{\rho}_{1}\left\{\boldsymbol{\kappa}_{n_{K}}\left[\lambda_{1}, \lambda_{2}, \ldots \lambda_{n_{K}}\right] \mid \mathrm{v}\right\}, \quad \lambda_{1}, \ldots, \lambda_{n} \in K, \mathbf{v} \in \mathrm{V}
$$

In the general case of $k_{\rho}$-place actions, the multiplication in the $n_{\rho}$-ary semigroup $\mathcal{S}_{\rho}$ can be defined by the changing arity formula DUPLIJ [2012] (schematically)

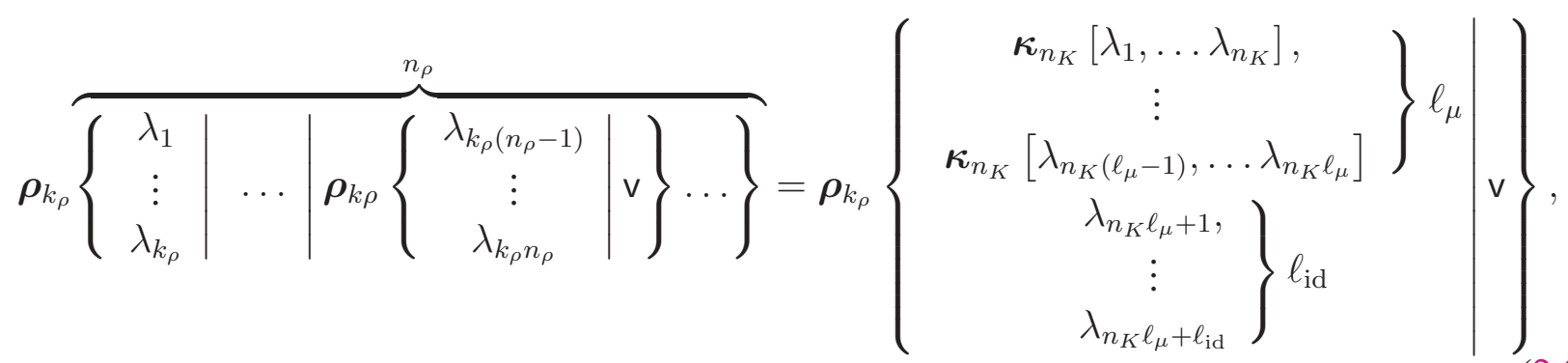

where $\ell_{\mu}$ and $\ell_{\text {id }}$ are both integers. The associativity of (2.9) in each concrete case can be achieved by applying the associativity quiver concept from DUPLIJ [2012].

Definition 2.1. The $\ell$-shape is a pair $\left(\ell_{\mu}, \ell_{\mathrm{id}}\right)$, where $\ell_{\mu}$ is the number of multiplications and $\ell_{\mathrm{id}}$ is the number of intact elements in the composition of operations.

It follows from (2.9),

Proposition 2.2. The arities of the polyadic field $\mathbb{K}_{m_{K}, n_{K}}$, the arity $n_{\rho}$ of the multiaction semigroup $\mathrm{S}_{\rho}$ and the $\ell$-shape of the composition satisfy

$$
\begin{gathered}
k_{\rho} n_{\rho}=n_{K} \ell_{\mu}+\ell_{\mathrm{id}}, \\
k_{\rho}=\ell_{\mu}+\ell_{\mathrm{id}} .
\end{gathered}
$$

We can exclude $\ell_{\mu}$ or $\ell_{\text {id }}$ and obtain

$$
n_{\rho}=n_{K}-\frac{n_{K}-1}{k_{\rho}} \ell_{\mathrm{id}}, \quad n_{\rho}=\frac{n_{K}-1}{k_{\rho}} \ell_{\mu}+1,
$$

respectively, where $\frac{n_{K}-1}{k_{\rho}} \ell_{\mathrm{id}} \geq 1$ and $\frac{n_{K}-1}{k_{\rho}} \ell_{\mu} \geq 1$ are integers. The following inequalities hold

$$
1 \leq \ell_{\mu} \leq k_{\rho}, \quad 0 \leq \ell_{\mathrm{id}} \leq k_{\rho}-1, \quad \ell_{\mu} \leq k_{\rho} \leq\left(n_{K}-1\right) \ell_{\mu}, \quad 2 \leq n_{\rho} \leq n_{K} .
$$

Remark 2.3. The formulas (2.12) coincide with the arity changing formulas for heteromorphisms DUPLIJ [2012] applied to (2.9).

It follows from (2.10), that the $\ell$-shape is determined by the arities and number of places $k_{\rho}$ by

$$
\ell_{\mu}=\frac{k_{\rho}\left(n_{\rho}-1\right)}{n_{K}-1}, \quad \ell_{\mathrm{id}}=\frac{k_{\rho}\left(n_{K}-n_{\rho}\right)}{n_{K}-1} .
$$

Because we have two polyadic "additions" $\nu_{m_{V}}$ and $\boldsymbol{\sigma}_{m_{K}}$, we need to consider, how the multiaction $\boldsymbol{\rho}_{k_{\rho}}$ "distributes" between each of them. First, consider distributivity of the multiaction $\boldsymbol{\rho}_{k_{\rho}}$ with 
respect to "vector addition" $\boldsymbol{\nu}_{m_{V}}$ (a polyadic analog of the binary $\lambda(\mathrm{v}+\mathrm{u})=\lambda \mathrm{v}+\lambda \mathrm{u}, \mathrm{v}, \mathrm{u} \in \mathrm{V}$, $\lambda, \mu \in K)$

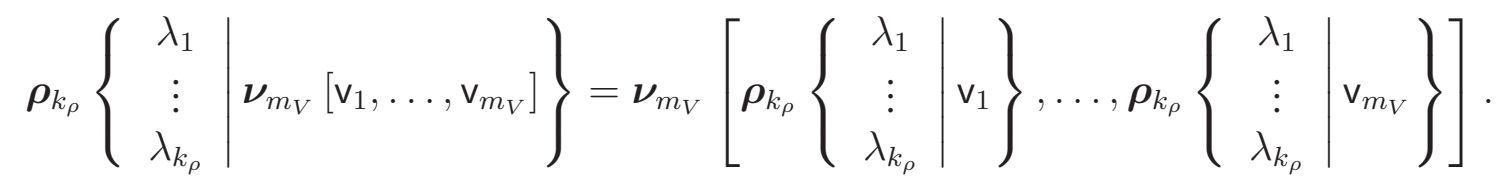

Observe that here, in distinction to (2.9), there is no connection between the arities $m_{V}$ and $k_{\rho}$.

Secondly, the distributivity of the multiaction $\boldsymbol{\rho}_{k_{\rho}}$ ("multiplication by scalar") with respect to the "field addition" (a polyadic analog of $(\lambda+\mu) v=\lambda v+\mu v, v \in A, \lambda, \mu \in K$ ) has a form similar to (2.9) (which can be obtained from the changing arity formula DUPLIJ [2012])

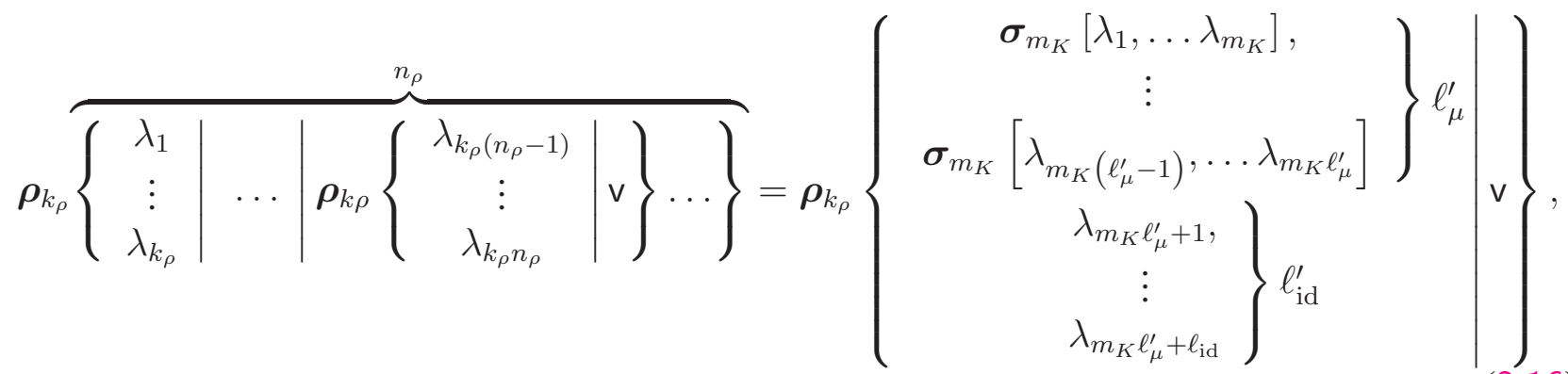

where $\ell_{\rho}^{\prime}$ and $\ell_{\mathrm{id}}^{\prime}$ are the numbers of multiplications and intact elements in the resulting multiaction, respectively. Here the arities are not independent as in (2.15), and so we have

Proposition 2.4. The arities of the polyadic field $\mathbb{K}_{m_{K}, n_{K}}$, the arity $n_{\rho}$ of the multiaction semigroup $\mathcal{S}_{\rho}$ and the $\ell$-shape of the distributivity satisfy

$$
\begin{aligned}
k_{\rho} n_{\rho} & =m_{K} \ell_{\mu}^{\prime}+\ell_{\mathrm{id}}^{\prime}, \\
k_{\rho} & =\ell_{\mu}^{\prime}+\ell_{\mathrm{id}}^{\prime} .
\end{aligned}
$$

It follows from (2.17)-(2.18)

$$
n_{\rho}=m_{K}-\frac{m_{K}-1}{k_{\rho}} \ell_{\mathrm{id}}^{\prime}, \quad n_{\rho}=\frac{m_{K}-1}{k_{\rho}} \ell_{\mu}^{\prime}+1 .
$$

Here $\frac{m_{K}-1}{k_{\rho}} \ell_{\mathrm{id}}^{\prime} \geq 1$ and $\frac{m_{K}-1}{k_{\rho}} \ell_{\mu}^{\prime} \geq 1$ are integers, and we have the inequalities

$$
1 \leq \ell_{\mu}^{\prime} \leq k_{\rho}, \quad 0 \leq \ell_{\mathrm{id}}^{\prime} \leq k_{\rho}-1, \quad \ell_{\mu}^{\prime} \leq k_{\rho} \leq\left(m_{K}-1\right) \ell_{\mu}^{\prime}, \quad 2 \leq n_{\rho} \leq m_{K} .
$$

Now, the $\ell$-shape of the distributivity is fully determined from the arities and number of places $k_{\rho}$ by the arity shape formulas

$$
\ell_{\rho}^{\prime}=\frac{k_{\rho}\left(n_{\rho}-1\right)}{m_{K}-1}, \quad \ell_{\mathrm{id}}^{\prime}=\frac{k_{\rho}\left(m_{K}-n_{\rho}\right)}{m_{K}-1} .
$$

It follows from (2.20) that:

Corollary 2.5. The arity $n_{\rho}$ of the multiaction semigroup $\mathcal{S}_{\rho}$ is less than or equal to the arity of the field addition $m_{K}$.

Definition 2.6. A polyadic ( $\mathbb{K}$ )-vector ("linear") space over a polyadic field is the 2-set 4-operation algebraic structure

$$
\mathcal{V}_{m_{K}, n_{K}, m_{V}, k_{\rho}}=\left\langle K ; \mathrm{V}\left|\boldsymbol{\sigma}_{m_{K}}, \boldsymbol{\kappa}_{n_{K}} ; \boldsymbol{\nu}_{m_{V}}\right| \boldsymbol{\rho}_{k_{\rho}}\right\rangle
$$

such that the following axioms hold:

1) $\left\langle K \mid \boldsymbol{\sigma}_{m_{K}}, \boldsymbol{\kappa}_{n_{K}}\right\rangle$ is a polyadic $\left(m_{K}, n_{K}\right)$-field $\mathbb{K}_{m_{K}, n_{K}}$;

2) $\left\langle A \mid \boldsymbol{\nu}_{m_{V}}\right\rangle$ is a commutative $m_{V}$-ary group; 
3) $\left\langle\boldsymbol{\rho}_{k_{\rho}}\right|$ composition $\rangle$ is a $n_{\rho}$-ary semigroup $\mathrm{S}_{\rho}$;

4) Distributivity of the multiaction $\boldsymbol{\rho}_{k_{\rho}}$ with respect to the "vector addition" $\boldsymbol{\nu}_{m_{V}}(2.15)$;

5) Distributivity of $\boldsymbol{\rho}_{k_{\rho}}$ with respect to the "scalar addition" $\boldsymbol{\sigma}_{m_{K}}$ (2.16);

6) Compatibility of $\boldsymbol{\rho}_{k_{\rho}}$ with the "scalar multiplication" $\boldsymbol{\kappa}_{n_{K}}(2.9)$;

7) Normalization of the multiaction $\boldsymbol{\rho}_{k_{\rho}}(2.6)$.

All of the arities in (2.22) are independent and can be chosen arbitrarily, but they fix the $\ell$-shape of the multiaction composition (2.9) and the distributivity (2.16) by (2.14) and (2.21), respectively. Note that the main distinction from the binary case is the possibility for the arity $n_{\rho}$ of the multiaction semigroup $\mathcal{S}_{\rho}$ to be arbitrary.

Definition 2.7. A polyadic $\mathbb{K}$-vector subspace is

$$
\mathcal{V}_{m_{K}, n_{K}, m_{V}, k_{\rho}}^{s u b}=\left\langle K ; \mathrm{V}^{s u b}\left|\boldsymbol{\sigma}_{m_{K}}, \boldsymbol{\kappa}_{n_{K}} ; \boldsymbol{\nu}_{m_{V}}\right| \boldsymbol{\rho}_{k_{\rho}}\right\rangle,
$$

where the subset $\mathrm{V}^{\text {sub }} \subset \mathrm{V}$ is closed under all operations $\boldsymbol{\sigma}_{m_{K}}, \boldsymbol{\kappa}_{n_{K}}, \boldsymbol{\nu}_{m_{V}}, \boldsymbol{\rho}_{k_{\rho}}$ and the axioms 1)-7).

Let us consider a subset $\mathrm{S}=\left\{\mathrm{v}_{1}, \ldots, \mathrm{v}_{d_{V}}\right\} \subseteq \mathrm{V}$ (of $d_{V}$ "vectors"), then a polyadic span of $\mathrm{S}$ is (a "linear combination")

$$
\begin{aligned}
& \operatorname{Span}_{\text {pol }}^{\lambda}\left(\mathrm{v}_{1}, \ldots, \mathrm{v}_{d_{V}}\right)=\{w\}, \\
& w=\nu_{m_{V}}^{\ell_{\nu}}\left[\boldsymbol{\rho}_{k_{\rho}}\left\{\begin{array}{c|c}
\lambda_{1} & \\
\vdots & \mathrm{v}_{1} \\
\lambda_{k_{\rho}} &
\end{array}\right\}, \ldots, \boldsymbol{\rho}_{k_{\rho}}\left\{\begin{array}{c|c}
\lambda_{\left(d_{V}-1\right) k_{\rho}} & \\
\vdots & \mathrm{v}_{s} \\
\lambda_{d_{V} k_{\rho}} &
\end{array}\right\}\right],
\end{aligned}
$$

where $\left(d_{V} \cdot k_{\rho}\right)$ "scalars" play the role of coefficients (or coordinates as columns consisting of $k_{\rho}$ elements from the polyadic field $\mathbb{K}_{m_{K}, n_{K}}$ ), and the number of "vectors" $s$ is connected with the "number of $m_{V}$-ary additions" $\ell_{\nu}$ by

$$
d_{V}=\ell_{\nu}\left(m_{V}-1\right)+1,
$$

while $\operatorname{Span}_{\text {pol }}^{\lambda} S$ is the set of all "vectors" of this form (2.24) (we consider here finite "sums" only).

Definition 2.8. A polyadic span $\mathrm{S}=\left\{\mathrm{v}_{1}, \ldots, \mathrm{v}_{d_{V}}\right\} \subseteq \mathrm{V}$ is nontrivial, if at least one multiaction $\boldsymbol{\rho}_{k_{\rho}}$ in (2.24) is nonzero.

Since polyadic fields and groups may not contain zeroes, we need to redefine the basic notions of equivalences. Let us take two different spans of the same set $\mathrm{S}$.

Definition 2.9. A set $\left\{\mathrm{v}_{1}, \ldots, \mathrm{v}_{d_{V}}\right\}$ is called "linear" polyadic independent, if from the equality of nontrivial spans, as $\operatorname{Span}_{\text {pol }}^{\lambda}\left(\mathrm{v}_{1}, \ldots, \mathrm{v}_{d_{V}}\right)=\operatorname{Span}_{\text {pol }}^{\lambda^{\prime}}\left(\mathrm{v}_{1}, \ldots, \mathrm{v}_{d_{V}}\right)$, it follows that all $\lambda_{i}=\lambda_{i}^{\prime}, i=$ $1, \ldots, d_{V} k_{\rho}$.

Definition 2.10. A set $\left\{\mathrm{v}_{1}, \ldots, \mathrm{v}_{d_{V}}\right\}$ is called a polyadic basis of a polyadic vector space $\mathcal{V}_{m_{K} n_{K} m_{V} k_{\rho}}$, if it spans the whole space $\operatorname{Span}_{\text {pol }}^{\lambda}\left(\mathrm{v}_{1}, \ldots, \mathrm{v}_{d_{V}}\right)=\mathrm{V}$.

In other words, any element of $\mathrm{V}$ can be uniquely presented in the form of the polyadic "linear combination" (2.24). If a polyadic vector space $\mathcal{V}_{m_{K} n_{K} m_{V} k_{\rho}}$ has a finite basis $\left\{\mathrm{v}_{1}, \ldots, \mathrm{v}_{d_{V}}\right\}$, then any another basis $\left\{\mathrm{v}_{1}^{\prime}, \ldots, \mathrm{v}_{d_{V}}^{\prime}\right\}$ has the same number of elements.

Definition 2.11. The number of elements in the polyadic basis $\left\{\mathrm{v}_{1}, \ldots, \mathrm{v}_{d_{V}}\right\}$ is called the polyadic dimension of $\mathcal{V}_{m_{K}, n_{K}, m_{V}, k_{\rho}}$.

Remark 2.12. The so-called 3 -vector space introduced and studied in DUPLIJ AND WERNER [2015], corresponds to $\mathcal{V}_{m_{K}=3, n_{K}=2, m_{V}=3, k_{\rho}=1}$. 
2.2. One-set polyadic vector space. A particular polyadic vector space is important: consider $\mathrm{V}=$ $K, \boldsymbol{\nu}_{m_{V}}=\boldsymbol{\sigma}_{m_{K}}$ and $m_{V}=m_{K}$, which gives the following one-set "linear" algebraic structure (we call it a one-set polyadic vector space)

$$
\mathcal{K}_{m_{K}, n_{K}, k_{\rho}}=\left\langle K\left|\boldsymbol{\sigma}_{m_{K}}, \boldsymbol{\kappa}_{n_{K}}\right| \boldsymbol{\rho}_{k_{\rho}}^{\lambda}\right\rangle
$$

where now the multiaction

$$
\boldsymbol{\rho}_{k_{\rho}}^{\lambda}\left\{\begin{array}{c|c}
\lambda_{1} \\
\vdots \\
\lambda_{k_{\rho}}
\end{array} \mid \lambda\right\}, \lambda, \lambda_{i} \in K
$$

acts on $K$ itself (in some special way), and therefore can be called a regular multiaction. In the binary case $n_{K}=m_{K}=2$, the only possibility for the regular action is the multiplication (by "scalars") in the field $\rho_{1}^{\lambda}\left\{\lambda_{1} \mid \lambda\right\}=\kappa_{2}\left[\lambda_{1} \lambda\right]\left(\equiv \lambda_{1} \lambda\right)$, which obviously satisfies the axioms 4)-7) of a vector space in Definition 2.6. In this way we arrive at the definition of the binary field $\mathbb{K} \equiv \mathbb{K}_{2,2}=\left\langle K \mid \boldsymbol{\sigma}_{2}, \boldsymbol{\kappa}_{2}\right\rangle$, and so a one-set binary vector space coincides with the underlying field $\mathcal{K}_{m_{K}=2, n_{K}=2, k_{\rho}=1}=\mathbb{K}$, or as it is said "a field is a (one-dimensional) vector space over itself".

Remark 2.13. In the polyadic case, the regular multiaction $\boldsymbol{\rho}_{k_{\rho}}^{\lambda}$ can be chosen, as any (additional to $\boldsymbol{\sigma}_{m_{K}}, \boldsymbol{\kappa}_{n_{K}}$ ) function satisfying axioms 4)-7) of a polyadic vector space and the number of places $k_{\rho}$ and the arity of the semigroup of multiactions $\mathcal{S}_{\rho}$ can be arbitrary, in general. Also, $\rho_{k_{\rho}}^{\lambda}$ can be taken as a some nontrivial combination of $\boldsymbol{\sigma}_{m_{K}}, \boldsymbol{\kappa}_{n_{K}}$ satisfying axioms 4)-7) (which admits a nontrivial "multiplication by scalars").

In the simplest regular (similar to the binary) case,

$$
\boldsymbol{\rho}_{k_{\rho}}^{\lambda}\left\{\begin{array}{c|c}
\lambda_{1} & \\
\vdots & \lambda \\
\lambda_{k_{\rho}} &
\end{array}\right\}=\boldsymbol{\kappa}_{n_{K}}^{\ell_{\kappa}}\left[\lambda_{1}, \ldots, \lambda_{k_{\rho}}, \lambda\right],
$$

where $\ell_{\kappa}$ is the number of multiplications $\kappa_{n_{K}}$, and the number of places $k_{\rho}$ is now fixed by

$$
k_{\rho}=\ell_{\kappa}\left(n_{K}-1\right),
$$

while $\lambda$ in (2.29) can be on any place due the commutativity of the field multiplication $\kappa_{n_{K}}$.

Remark 2.14. In general, the one-set polyadic vector space need not coincide with the underlying polyadic field, $\mathcal{K}_{m_{K}, n_{K}, k_{\rho}} \neq \mathbb{K}_{n_{K} m_{K}}$ (as opposed to the binary case), but can have a more complicated structure which is determined by an additional multiplace function, the multiaction $\rho_{k_{\rho}}^{\lambda}$.

2.3. Polyadic algebras. By analogy with the binary case, introducing an additional operation on vectors, a multiplication which is distributive and "linear" with respect to "scalars", leads to a polyadic generalization of the (associative) algebra notion CARLSSON [1980]. Here, we denote the second (except for the 'scalars' $K$ ) set by $\mathrm{A}$ (instead of $\mathrm{V}$ above), on which we define $t$ wo operations: $m_{A}$-ary "addition" $\boldsymbol{\nu}_{m_{A}}: \mathrm{A}^{\times m_{A}} \rightarrow$ A and $n_{A}$-ary "multiplication" $\boldsymbol{\mu}_{n_{A}}: \mathrm{A}^{\times n_{A}} \rightarrow$ A. To interpret the $n_{A^{-}}$-ary operation as a true multiplication, the operations $\boldsymbol{\mu}_{n_{A}}$ and $\boldsymbol{\nu}_{m_{A}}$ should satisfy polyadic distributivity (1.1)-(1.3) (an analog of $(a+b) \cdot c=a \cdot c+b \cdot c$, with $a, b, c \in A)$. Then we should consider the "interaction" of this new operation $\boldsymbol{\mu}_{n_{A}}$ with the multiaction $\boldsymbol{\rho}_{k_{\rho}}$ (an analog of the "compatibility with scalars" $(\lambda \mathrm{a}) \cdot(\mu \mathrm{b})=(\lambda \mu) \mathrm{a} \cdot \mathrm{b}, \mathrm{a}, \mathrm{b} \in \mathrm{A}, \lambda, \mu \in K)$. In the most general case, when all arities are 
arbitrary, we have the polyadic compatibility of $\boldsymbol{\mu}_{n_{A}}$ with the field multiplication $\kappa_{n_{K}}$ as follows

$$
\begin{aligned}
& \boldsymbol{\mu}_{n_{A}}\left[\boldsymbol{\rho}_{k_{\rho}}\left\{\begin{array}{c|c}
\lambda_{1} & \\
\vdots & \mathrm{a}_{1} \\
\lambda_{k_{\rho}} &
\end{array}\right\}, \ldots, \boldsymbol{\rho}_{k_{\rho}}\left\{\begin{array}{c}
\lambda_{k_{\rho}\left(n_{A}-1\right)} \\
\vdots \\
\lambda_{k_{\rho} n_{A}}
\end{array} \mid \mathrm{a}_{n_{A}}\right\}\right] \\
& \left.\left.=\boldsymbol{\rho}_{k_{\rho}}\left\{\begin{array}{c}
\boldsymbol{\kappa}_{n_{K}}\left[\lambda_{1}, \ldots, \lambda_{n_{K}}\right], \\
\vdots \\
\boldsymbol{\kappa}_{n_{K}}\left[\lambda_{n_{K}\left(\ell_{\mu}^{\prime \prime}-1\right)}, \ldots, \lambda_{n_{K} \ell_{\mu}^{\prime \prime}}\right] \\
\lambda_{n_{K} \ell_{\mu}^{\prime \prime}+1} \\
\vdots \\
\lambda_{n_{K} \ell_{\mu}^{\prime \prime}+\ell_{\mathrm{id}}^{\prime \prime}}
\end{array}\right\} \ell_{\mathrm{id}}^{\prime \prime}\left|\boldsymbol{\mu}_{\mu}^{\prime \prime}\right| \mathrm{a}_{n_{A}} \mid \mathrm{a}_{n_{A}}\right]\right\},
\end{aligned}
$$

where $\ell_{\mu}^{\prime \prime}$ and $\ell_{\mathrm{id}}^{\prime \prime}$ are the numbers of multiplications and intact elements in the resulting multiaction, respectively.

Proposition 2.15. The arities of the polyadic field $\mathbb{K}_{m_{K}, n_{K}}$, the arity $n_{\rho}$ of the multiaction semigroup $\mathrm{S}_{\rho}$ and the $\ell$-shape of the polyadic compatibility (2.31) satisfy

$$
k_{\rho} n_{A}=n_{K} \ell_{\mu}^{\prime \prime}+\ell_{\mathrm{id}}^{\prime \prime}, \quad k_{\rho}=\ell_{\mu}^{\prime \prime}+\ell_{\mathrm{id}}^{\prime \prime} .
$$

We can exclude from (2.32) $\ell_{\rho}^{\prime \prime}$ or $\ell_{\mathrm{id}}^{\prime \prime}$ and obtain

$$
n_{A}=n_{K}-\frac{n_{K}-1}{k_{\rho}} \ell_{\mathrm{id}}^{\prime \prime}, \quad n_{A}=\frac{n_{K}-1}{k_{\rho}} \ell_{\mu}^{\prime \prime}+1,
$$

where $\frac{n_{K}-1}{k_{\rho}} \ell_{\mathrm{id}}^{\prime \prime} \geq 1$ and $\frac{n_{K}-1}{k_{\rho}} \ell_{\mu}^{\prime \prime} \geq 1$ are integer, and the inequalities hold

$$
1 \leq \ell_{\mu}^{\prime \prime} \leq k_{\rho}, \quad 0 \leq \ell_{\mathrm{id}}^{\prime \prime} \leq k_{\rho}-1, \quad \ell_{\mu}^{\prime \prime} \leq k_{\rho} \leq\left(n_{K}-1\right) \ell_{\mu}^{\prime \prime}, \quad 2 \leq n_{A} \leq n_{K}
$$

It follows from (2.32), that the $\ell$-shape is determined by the arities and number of places $k_{\rho}$ as

$$
\ell_{\mu}^{\prime \prime}=\frac{k_{\rho}\left(n_{A}-1\right)}{n_{K}-1}, \quad \ell_{\mathrm{id}}^{\prime \prime}=\frac{k_{\rho}\left(n_{K}-n_{A}\right)}{n_{K}-1} .
$$

Definition 2.16. A polyadic ("linear") algebra over a polyadic field is the 2-set 5-operation algebraic structure

$$
\mathcal{A}_{m_{K}, n_{K}, m_{A}, n_{A}, k_{\rho}}=\left\langle K ; \mathrm{A}\left|\boldsymbol{\sigma}_{m_{K}}, \boldsymbol{\kappa}_{n_{K}} ; \boldsymbol{\nu}_{m_{A}}, \boldsymbol{\mu}_{n_{A}}\right| \boldsymbol{\rho}_{k_{\rho}}\right\rangle,
$$

such that the following axioms hold:

1) $\left\langle K\right.$; A $\left.\left|\boldsymbol{\sigma}_{m_{K}}, \boldsymbol{\kappa}_{n_{K}} ; \boldsymbol{\nu}_{m_{A}}\right| \boldsymbol{\rho}_{k_{\rho}}\right\rangle$ is a polyadic vector space over a polyadic field $\mathbb{K}_{m_{K}, n_{K}}$;

2) The algebra multiplication $\boldsymbol{\mu}_{n_{A}}$ and the algebra addition $\boldsymbol{\nu}_{m_{A}}$ satisfy the polyadic distributivity (1.1)-(1.3);

3) The multiplications in the algebra $\boldsymbol{\mu}_{n_{A}}$ and in the field $\kappa_{n_{K}}$ are compatible by (2.31).

In the case where the algebra multiplication $\boldsymbol{\mu}_{n_{\Lambda}}$ is associative (1.4), then $\mathcal{A}_{m_{K}, n_{K}, m_{A}, n_{A}, k_{\rho}}$ is an associative polyadic algebra (for $k_{\rho}=1$ see CARLSSON [1980]). If $\boldsymbol{\mu}_{n_{A}}$ is commutative, $\mu_{n_{A}}\left[\boldsymbol{a}_{A}\right]=$ $\mu_{n_{A}}\left[\sigma \circ \boldsymbol{a}_{A}\right]$, for any polyad in algebra $\boldsymbol{a}_{A} \in \mathrm{A}^{\times n_{A}}$ for all permutations $\sigma \in S_{n}$, where $S_{n}$ is the symmetry group, then $\mathcal{A}_{m_{K}, n_{K}, m_{A}, n_{A}, k_{\rho}}$ is called a commutative polyadic algebra. As in the $n$-ary (semi)group theory, for polyadic algebras one can introduce special kinds of associativity and partial commutativity. If the multiplication $\boldsymbol{\mu}_{n_{A}}$ contains the identity $\mathrm{e}_{A}$ (1.6) or a neutral polyad for any element, such a polyadic algebra is called unital or neutral-unital, respectively. It follows from (2.34) that:

Corollary 2.17. In a polyadic ("linear") algebra the arity of the algebra multiplication $n_{A}$ is less than or equal to the arity of the field multiplication $n_{K}$. 
Proposition 2.18. It all the operation $\ell-$ shapes in (2.9), (2.16) and (2.31) coincide

$$
\ell_{\mu}^{\prime \prime}=\ell_{\mu}^{\prime}=\ell_{\mu}, \quad \ell_{\mathrm{id}}^{\prime \prime}=\ell_{\mathrm{id}}^{\prime}=\ell_{\mathrm{id}},
$$

then, we obtain the conditions for the arities

$$
n_{K}=m_{K}, \quad n_{\rho}=n_{A},
$$

while $m_{A}$ and $k_{\rho}$ are not connected.

Proof. Use (2.14) and (2.35).

Proposition 2.19. In the case of equal $\ell$-shapes the multiplication and addition of the ground polyadic field ("scalars") should coincide, while the arity $n_{\rho}$ of the multiaction semigroup $\mathcal{S}_{\rho}$ should be the same as of the algebra multiplication $n_{A}$, while the arity of the algebra addition $m_{A}$ and number of places $k_{\rho}$ remain arbitrary.

Remark 2.20. The above $\ell$-shapes (2.14), (2.21), and (2.35) are defined by a pair of integers, and therefore the arities in them are not arbitrary, but should be "quantized" in the same manner as the arities of heteromorphisms in DUPLIJ [2012].

Therefore, numerically the "quantization" rules for the $\ell$-shapes (2.14), (2.21), and (2.35) coincide and given in TABLE 1 .

\begin{tabular}{|c|c|c|c|c|}
\hline$k_{\rho}$ & $\ell_{\mu}\left|\ell_{\mu}^{\prime}\right| \ell_{\mu}^{\prime \prime}$ & $\ell_{\mathrm{id}}\left|\ell_{\mathrm{id}}^{\prime}\right| \ell_{\mathrm{id}}^{\prime \prime}$ & \begin{tabular}{c|c}
$n_{K}$ & $m_{K}$ \\
$n_{\rho}$ & $n_{\rho}$ \\
\end{tabular} & $\begin{array}{l}n_{K} \\
n_{A}\end{array}$ \\
\hline 2 & 1 & 1 & $\begin{array}{lll}3, & 5, & 7, \\
2, & 3, & 4,\end{array}$ & $\begin{array}{l}\ldots \\
\ldots\end{array}$ \\
\hline 3 & 1 & 2 & 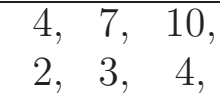 & $\begin{array}{l}\cdots \\
\cdots\end{array}$ \\
\hline 3 & 2 & 1 & 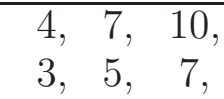 & $\begin{array}{l} \\
\cdots\end{array}$ \\
\hline 4 & 1 & 3 & $\begin{array}{ccc}5, & 9, & 13, \\
2, & 3, & 4,\end{array}$ & $\begin{array}{l}\cdots \\
\cdots\end{array}$ \\
\hline 4 & 2 & 2 & $\begin{array}{lll}3, & 5, & 7, \\
2, & 3, & 4,\end{array}$ & $\begin{array}{l}\cdots \\
\cdots\end{array}$ \\
\hline 4 & 3 & 1 & $\begin{array}{lll}5, & 9, & 13, \\
4, & 7, & 10,\end{array}$ & $\begin{array}{l}\ldots \\
\ldots\end{array}$ \\
\hline
\end{tabular}

TABLE 1. "Quantization” of arity $\ell$-shapes

Thus, we arrive at the following

Theorem 2.21 (The arity partial freedom principle). The basic two-set polyadic algebraic structures have non-free underlying operation arities which are "quantized" in such a way that their $\ell$-shape is given by integers.

The above definitions can be generalized, as in the binary case by considering a polyadic ring $\mathcal{R}_{m_{K}, n_{K}}$ instead of a polyadic field $\mathbb{K}_{m_{K}, n_{K}}$. In this way a polyadic vector space becomes a polyadic module over a ring or polyadic $\mathcal{R}$-module, while a polyadic algebra over a polyadic field becomes a polyadic algebra over a ring or polyadic $\mathcal{R}$-algebra. All the axioms and relations between arities in the Definition 2.6 and Definition 2.16 remain the same. However, one should take into account that the ring multiplication $\boldsymbol{\kappa}_{n_{K}}$ can be noncommutative, and therefore for polyadic $\mathcal{R}$-modules and $\mathcal{R}$ algebras it is necessary to consider many different kinds of multiactions $\boldsymbol{\rho}_{k_{\rho}}$ (all of them are described in (2.9)). For instance, in the ternary case this corresponds to left, right and central ternary modules, and tri-modules CARLSSON [1976], BAZUNOVA ET AL. [2004]. 


\section{Mappings BetweEn POLYADIC ALGEBRAiC STRUCTURES}

Let us consider $D_{V}$ different polyadic vector spaces over the same polyadic field $\mathbb{K}_{m_{K}, n_{K}}$, as

$$
\mathcal{V}_{m_{K}, n_{K}, m_{V}^{(i)}, k_{\rho}^{(i)}}^{(i)}=\left\langle K ; \mathrm{V}^{(i)}\left|\boldsymbol{\sigma}_{m_{K}}, \boldsymbol{\kappa}_{n_{K}} ; \boldsymbol{\nu}_{m_{V}^{(i)}}^{(i)}\right| \boldsymbol{\rho}_{k_{\rho}^{(i)}}^{(i)}\right\rangle, \quad i=1, \ldots, D_{V}<\infty
$$

Here we define a polyadic analog of a "linear" mapping for polyadic vector spaces which "commutes" with the "vector addition" and the "multiplication by scalar" (an analog of the additivity $\boldsymbol{F}(\mathrm{v}+\mathrm{u})=\boldsymbol{F}(\mathrm{v})+\boldsymbol{F}(\mathrm{u})$, and the homogeneity of degree one $\boldsymbol{F}(\lambda \mathrm{v})=\lambda \boldsymbol{F}(\mathrm{v}), \mathrm{v}, \mathrm{u} \in \mathrm{V}, \lambda \in K)$.

Definition 3.1. A 1-place (" $\mathbb{K}$-linear") mapping between polyadic vector spaces $\mathcal{V}_{m_{K}, n_{K}, m_{V}, k_{\rho}}=$ $\left\langle K ; \mathrm{V}\left|\boldsymbol{\sigma}_{m_{K}}, \boldsymbol{\kappa}_{n_{K}} ; \boldsymbol{\nu}_{m_{V}}\right| \boldsymbol{\rho}_{k_{\rho}}\right\rangle$ and $\mathcal{V}_{m_{K}, n_{K}, m_{V}, k_{\rho}}=\left\langle K ; \mathrm{V}^{\prime}\left|\boldsymbol{\sigma}_{m_{K}}, \boldsymbol{\kappa}_{n_{K}} ; \boldsymbol{\nu}_{m_{V}}^{\prime}\right| \boldsymbol{\rho}_{k_{\rho}}^{\prime}\right\rangle$ over the same polyadic field $\mathbb{K}_{m_{K}, n_{K}}=\left\langle K \mid \boldsymbol{\sigma}_{m_{K}}, \boldsymbol{\kappa}_{n_{K}}\right\rangle$ is $\boldsymbol{F}_{1}: \mathrm{V} \rightarrow \mathrm{V}^{\prime}$, such that

$$
\begin{gathered}
\boldsymbol{F}_{1}\left(\boldsymbol{\nu}_{m_{V}}\left[\mathrm{v}_{1}, \ldots, \mathrm{v}_{m_{V}}\right]\right)=\boldsymbol{\nu}_{m_{V}}^{\prime}\left[\boldsymbol{F}_{1}\left(\mathrm{v}_{1}\right), \ldots, \boldsymbol{F}_{1}\left(\mathrm{v}_{m_{V}}\right)\right], \\
\boldsymbol{F}_{1}\left(\boldsymbol{\rho}_{k_{\rho}}\left\{\begin{array}{c|c}
\lambda_{1} \\
\vdots \\
\lambda_{k_{\rho}}
\end{array} \mid \mathrm{v}\right\}\right)=\boldsymbol{\rho}_{k_{\rho}}^{\prime}\left\{\begin{array}{c}
\lambda_{1} \\
\vdots \\
\lambda_{k_{\rho}}
\end{array} \mid \boldsymbol{F}_{1}(\mathrm{v})\right\},
\end{gathered}
$$

where $\mathrm{v}_{1}, \ldots, \mathrm{v}_{m_{V}}, \mathrm{v} \in \mathrm{V}, \lambda_{1}, \ldots, \lambda_{k_{\rho}} \in K$.

If $z_{V}$ is a "zero vector" in $V$ and $z_{V^{\prime}}$ is a "zero vector" in $V^{\prime}$ (see (2.2)), then it follows from (3.2)-(3.3), that $\boldsymbol{F}_{1}\left(\mathrm{z}_{V}\right)=\mathrm{z}_{V^{\prime}}$.

The initial and final arities of $\boldsymbol{\nu}_{m_{V}}$ ("vector addition") and the multiaction $\boldsymbol{\rho}_{k_{\rho}}$ ("multiplication by scalar") coincide, because $\boldsymbol{F}_{1}$ is a 1-place mapping (a linear homomorphism). In DUPLIJ [2012] multiplace mappings and corresponding heteromorphisms were introduced. The latter allow us to change arities $\left(m_{V} \rightarrow m_{V}^{\prime}, k_{\rho} \rightarrow k_{\rho}^{\prime}\right)$, which is the main difference between binary and polyadic mappings.

Definition 3.2. A $k_{F}$-place (" $\mathbb{K}$-linear") mapping between two polyadic vector spaces $\mathcal{V}_{m_{K}, n_{K}, m_{V}, k_{\rho}}=\left\langle K ; \mathrm{V}\left|\boldsymbol{\sigma}_{m_{K}}, \boldsymbol{\kappa}_{n_{K}} ; \boldsymbol{\nu}_{m_{V}}\right| \boldsymbol{\rho}_{k_{\rho}}\right\rangle$ and $\mathcal{V}_{m_{K}, n_{K}, m_{V}, k_{\rho}}=\left\langle K ; \mathrm{V}^{\prime}\left|\boldsymbol{\sigma}_{m_{K}}, \boldsymbol{\kappa}_{n_{K}} ; \boldsymbol{\nu}_{m_{V}^{\prime}}^{\prime}\right| \boldsymbol{\rho}_{k_{\rho}^{\prime}}^{\prime}\right\rangle$ over the same polyadic field $\mathbb{K}_{m_{K}, n_{K}}=\left\langle K \mid \boldsymbol{\sigma}_{m_{K}}, \boldsymbol{\kappa}_{n_{K}}\right\rangle$ is defined, if there exists $\boldsymbol{F}_{k_{F}}: \mathrm{V}^{\times k_{F}} \rightarrow \mathrm{V}^{\prime}$, such that

$$
\boldsymbol{F}_{k_{F}}\left(\begin{array}{c}
\boldsymbol{\nu}_{m_{V}}\left[\mathrm{v}_{1}, \ldots, \mathrm{v}_{m_{V}}\right] \\
\vdots \\
\boldsymbol{\nu}_{m_{V}}\left[\begin{array}{c}
\left.\mathrm{v}_{m_{V}\left(\ell_{\mu}^{k}-1\right)}, \ldots \mathrm{v}_{m_{V} \ell_{\mu}^{k}}\right] \\
\mathrm{v}_{m_{V} \ell_{\mu}^{k}+1}, \\
\vdots \\
\mathrm{v}_{m_{V} \ell_{\mu}^{k}+\ell_{\mathrm{id}}^{k}}
\end{array}\right\} \ell_{\mathrm{id}}^{k}
\end{array}\right\}=\boldsymbol{\nu}_{m_{V}^{\prime}}^{\prime}\left[\boldsymbol{F}_{k_{F}}\left(\begin{array}{c}
\mathrm{v}_{1} \\
\vdots \\
\mathrm{v}_{k_{F}}
\end{array}\right), \ldots, \boldsymbol{F}_{k_{F}}\left(\begin{array}{c}
\mathrm{v}_{k_{F}\left(m_{V}^{\prime}-1\right)} \\
\vdots \\
\mathrm{v}_{k_{F} m_{V}^{\prime}}
\end{array}\right)\right],
$$




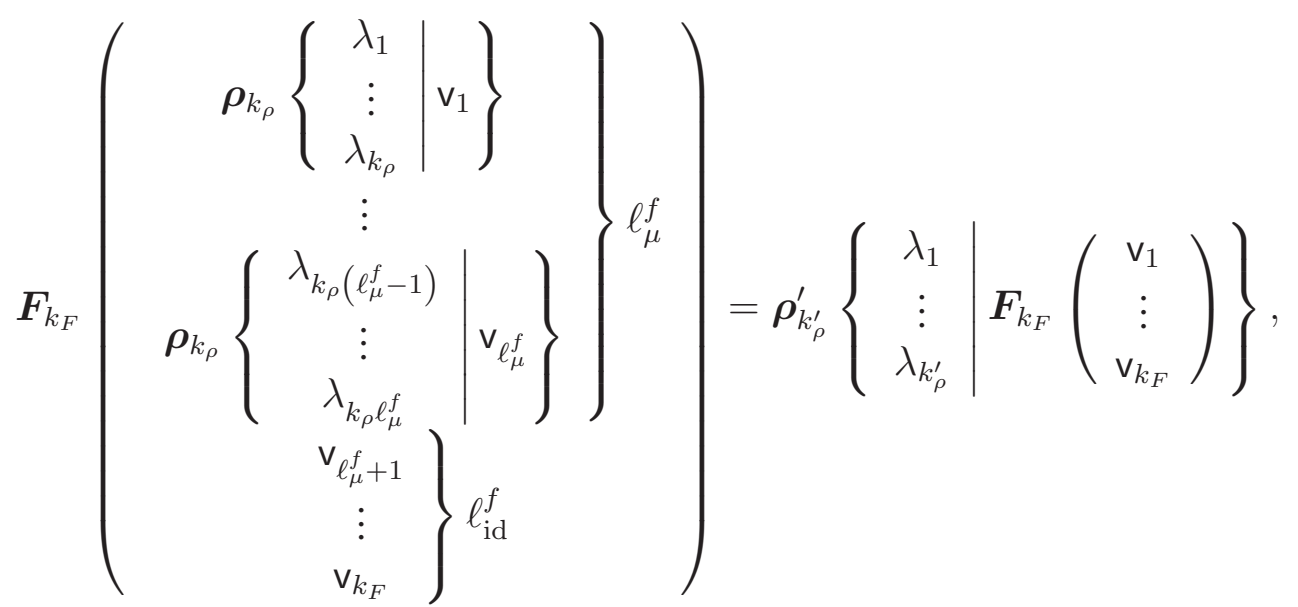

where $\mathrm{v}_{1}, \ldots, \mathrm{v}_{m_{V}}, \mathrm{v} \in \mathrm{V}, \lambda_{1}, \ldots, \lambda_{k_{\rho}} \in K$, and the four integers $\ell_{\rho}^{k}, \ell_{\mathrm{id}}^{k}, \ell_{\rho}^{f}, \ell_{\mathrm{id}}^{f}$ define the $\ell$-shape of the mapping.

It follows from (3.4)-(3.5), that the arities satisfy

$$
k_{F} m_{V}^{\prime}=m_{V} \ell_{\mu}^{k}+\ell_{\mathrm{id}}^{k}, \quad k_{F}=\ell_{\mu}^{k}+\ell_{\mathrm{id}}^{k}, \quad k_{F}=\ell_{\mu}^{f}+\ell_{\mathrm{id}}^{f}, \quad k_{\mu}^{\prime}=k_{\rho} \ell_{\mu}^{f} .
$$

The following inequalities hold

$$
1 \leq \ell_{\mu}^{k} \leq k_{F}, 0 \leq \ell_{\mathrm{id}}^{k} \leq k_{F}-1, \ell_{\mu}^{k} \leq k_{F} \leq\left(m_{V}-1\right) \ell_{\mu}^{k}, 2 \leq m_{V}^{\prime} \leq m_{V}, 2 \leq k_{\rho} \leq k_{\rho}^{\prime} .
$$

Thus, the $\ell$-shape of the $k_{F}$-place mapping between polyadic vector spaces is determined by

$$
\ell_{\mu}^{k}=\frac{k_{F}\left(m_{V}-1\right)}{m_{V}-1}, \quad \ell_{\mathrm{id}}^{k}=\frac{k_{F}\left(m_{V}-m_{V}^{\prime}\right)}{m_{V}-1}, \quad \ell_{\mu}^{f}=\frac{k_{\rho}}{k_{\rho}^{\prime}}, \quad \ell_{\mathrm{id}}^{f}=k_{F}-\frac{k_{\rho}}{k_{\rho}^{\prime}}
$$

3.1. Polyadic functionals and dual polyadic vector spaces. An important particular case of the $k_{F^{-}}$ place mapping can be considered, where the final polyadic vector space coincides with the underlying field (analog of a "linear functional").

Definition 3.3. A "linear" polyadic functional (or polyadic dual vector, polyadic covector) is a $k_{L^{-}}$ place mapping of a polyadic vector space $\mathcal{V}_{m_{K}, n_{K}, m_{V}, k_{\rho}}=\left\langle K ; \mathrm{V}\left|\boldsymbol{\sigma}_{m_{K}}, \boldsymbol{\kappa}_{n_{K}} ; \boldsymbol{\nu}_{m_{V}}\right| \boldsymbol{\rho}_{k_{\rho}}\right\rangle$ into its polyadic field $\mathbb{K}_{m_{K}, n_{K}}=\left\langle K \mid \boldsymbol{\sigma}_{m_{K}}, \boldsymbol{\kappa}_{n_{K}}\right\rangle$, such that there exists $L_{k_{L}}: \mathrm{V}^{\times k_{L}} \rightarrow K$, and

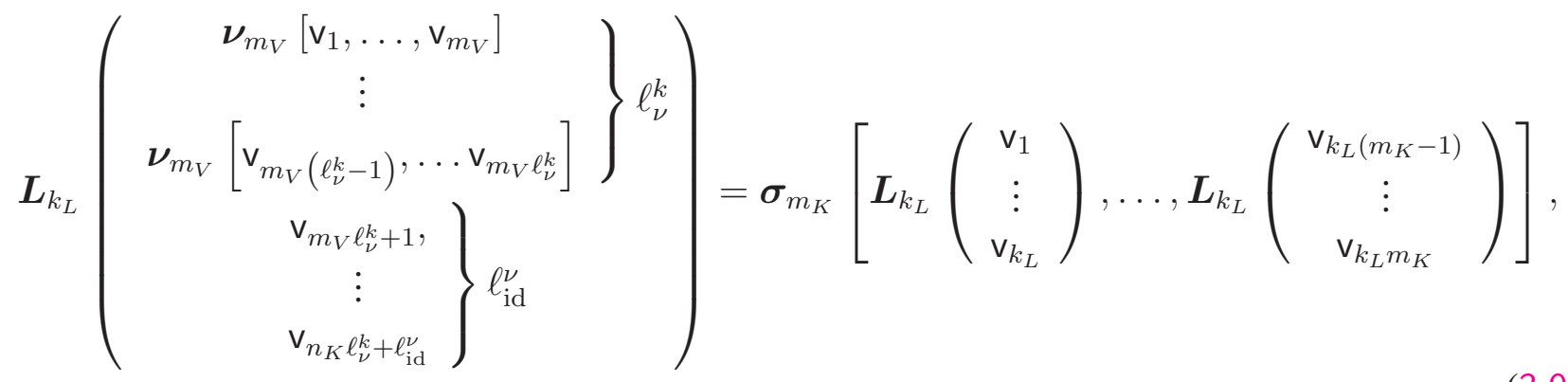




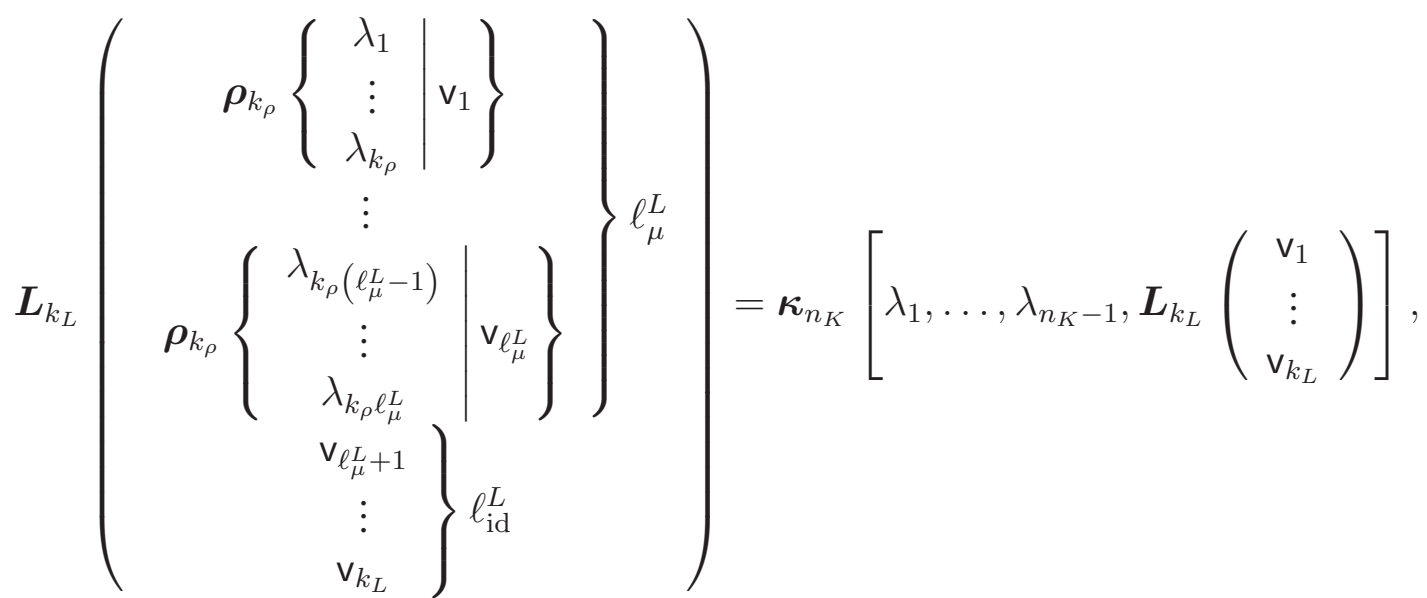

where $\mathrm{v}_{1}, \ldots, \mathrm{v}_{m_{V}}, \mathrm{v} \in V, \lambda_{1}, \ldots, \lambda_{n_{K}} \in K$, and the integers $\ell_{\nu}^{k}, \ell_{\mathrm{id}}^{\nu}, \ell_{\mu}^{L}, \ell_{\mathrm{id}}^{L}$ define the $\ell$-shape of $L_{k_{L}}$.

It follows from (3.4)-(3.5), that the arities satisfy

$$
k_{L} m_{K}=m_{V} \ell_{\nu}^{k}+\ell_{\mathrm{id}}^{\nu}, \quad k_{L}=\ell_{\nu}^{k}+\ell_{\mathrm{id}}^{\nu}, \quad k_{L}=\ell_{\mu}^{h}+\ell_{\mathrm{id}}^{h}, \quad n_{K}-1=k_{\rho} \ell_{\mu}^{h},
$$

and for them

$$
1 \leq \ell_{\nu}^{k} \leq k_{L}, 0 \leq \ell_{\mathrm{id}}^{\nu} \leq k_{L}-1, \ell_{\nu}^{k} \leq k_{L} \leq\left(m_{V}-1\right) \ell_{\nu}^{k}, 2 \leq m_{K} \leq m_{V}, 2 \leq k_{\rho} \leq n_{K}-1 .
$$

Thus, the $\ell$-shape of the polyadic functional is determined by

$$
\ell_{\nu}^{k}=\frac{k_{L}\left(m_{K}-1\right)}{m_{V}-1}, \quad \ell_{\mathrm{id}}^{\nu}=\frac{k_{L}\left(m_{V}-m_{K}\right)}{m_{V}-1}, \quad \ell_{\mu}^{h}=\frac{k_{\rho}}{n_{K}-1}, \quad \ell_{\mathrm{id}}^{h}=k_{L}-\frac{k_{\rho}}{n_{K}-1} .
$$

In the binary case, because the dual vectors (linear functionals) take their values in the underlying field, new operations between them, such that the dual vector "addition" $\left(+^{*}\right)$ and the "multiplication by a scalar" ( $\bullet *)$ can be naturally introduced by $\left(L^{(1)}+{ }^{*} L^{(2)}\right)(\mathrm{v})=L^{(1)}(\mathrm{v})+L^{(2)}(\mathrm{v}),(\lambda \bullet * L)(\mathrm{v})=$ $\lambda \bullet L(\mathrm{v})$, which leads to another vector space structure, called a dual vector space. Note that operations $+^{*}$ and,$+ \bullet^{*}$ and $\bullet$ are different, because + and $\bullet$ are the operations in the underlying field $\mathbb{K}$. In the polyadic case, we have more complicated arity changing formulas, and here we consider finitedimensional spaces only. The arities of operations between dual vectors can be different from ones in the underlying polyadic field $\mathbb{K}_{m_{K} n_{K}}$, in general. In this way, we arrive to the following

Definition 3.4. A polyadic dual vector space over a polyadic field $\mathbb{K}_{m_{K}, n_{K}}$ is

$$
\mathcal{V}_{m_{K}, n_{K}, m_{V}^{*}, k_{\rho}^{*}}^{*}=\left\langle K ;\left\{\boldsymbol{L}_{k_{L}}^{(i)}\right\}\left|\boldsymbol{\sigma}_{m_{K}}, \boldsymbol{\kappa}_{n_{K}} ; \boldsymbol{\nu}_{m_{L}}^{*}\right| \boldsymbol{\rho}_{k_{L}}^{*}\right\rangle,
$$

and the axioms are:

1) $\left\langle K \mid \boldsymbol{\sigma}_{m_{K}}, \boldsymbol{\kappa}_{n_{K}}\right\rangle$ is a polyadic $\left(m_{K}, n_{K}\right)$-field $\mathbb{K}_{m_{K}, n_{K}}$;

2) $\left\langle\left\{\boldsymbol{L}_{k_{L}}^{(i)}\right\} \mid \boldsymbol{\nu}_{m_{L}}^{*}, i=1, \ldots, D_{L}\right\rangle$ is a commutative $m_{L}$-ary group (which is finite, if $D_{L}<\infty$ );

3) The "dual vector addition" $\nu_{m_{L}}^{*}$ is compatible with the polyadic field addition $\boldsymbol{\sigma}_{m_{K}}$ by

$$
\boldsymbol{\nu}_{m_{L}}^{*}\left[\boldsymbol{L}_{k_{L}}^{(1)}, \ldots, \boldsymbol{L}_{k_{L}}^{\left(m_{L}\right)}\right]\left(\boldsymbol{a}^{\left(k_{L}\right)}\right)=\boldsymbol{\sigma}_{m_{K}}\left[\boldsymbol{L}_{k_{L}}^{(1)}\left(\boldsymbol{a}^{\left(k_{L}\right)}\right), \ldots, \boldsymbol{L}_{k_{L}}^{\left(m_{K}\right)}\left(\boldsymbol{v}^{\left(k_{L}\right)}\right)\right],
$$

where $\boldsymbol{v}^{\left(k_{L}\right)}=\left(\begin{array}{c}\mathrm{v}_{1} \\ \vdots \\ \mathrm{v}_{k_{L}}\end{array}\right), \mathrm{v}_{1}, \ldots, \mathrm{v}_{k_{L}} \in \mathrm{V}$, and it follows that

$$
m_{L}=m_{K}
$$


4) The compatibility of $\boldsymbol{\rho}_{k_{L}}^{*}$ with the "multiplication by a scalar" in the underlying polyadic field

$$
\boldsymbol{\rho}_{k_{L}}^{*}\left\{\begin{array}{c|c}
\lambda_{1} \\
\vdots & \boldsymbol{L}_{k_{L}} \\
\lambda_{k_{L}} &
\end{array}\right\}\left(\boldsymbol{v}^{\left(k_{L}\right)}\right)=\boldsymbol{\kappa}_{n_{K}}\left[\lambda_{1}, \ldots, \lambda_{n_{K}-1}, \boldsymbol{L}_{k_{L}}\left(\boldsymbol{v}^{\left(k_{L}\right)}\right)\right],
$$

and then

$$
k_{L}=n_{K}-1
$$

5) $\left\langle\left\{\boldsymbol{\rho}_{k_{L}}^{*}\right\}\right|$ composition $\rangle$ is a $n_{L}$-ary semigroup $\mathcal{S}_{L}$ (similar to (2.9))

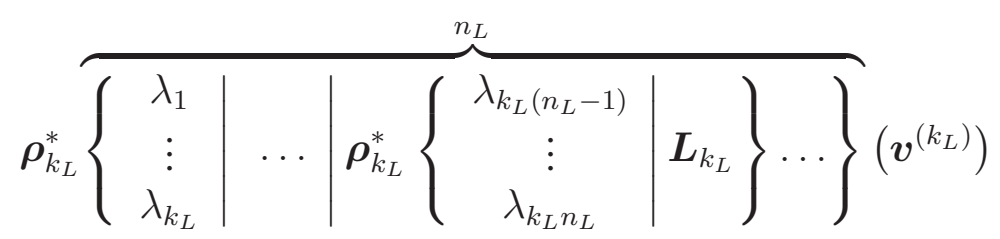

$$
\begin{aligned}
& =\boldsymbol{\rho}_{k_{L}}^{*}\left\{\begin{array}{c}
\boldsymbol{\kappa}_{n_{K}}\left[\lambda_{1}, \ldots \lambda_{n_{K}}\right] \\
\vdots \\
\boldsymbol{\kappa}_{n_{K}}\left[\lambda_{n_{K}\left(\ell_{\mu}^{L}-1\right)}, \ldots \lambda_{n_{K} \ell_{\mu}^{L}}\right] \\
\lambda_{n_{K} \ell_{\mu}^{L}+1} \\
\vdots \\
\lambda_{n_{K} \ell_{\mu}^{L}+\ell_{\mathrm{id}}^{L}}
\end{array}\right\} \ell^{L}\left|\ell^{L}\right| \boldsymbol{L}_{k_{L}}^{L} \mid\left(\boldsymbol{v}^{\left(k_{L}\right)}\right)
\end{aligned}
$$

where the $\ell$-shape is determined by the system

$$
k_{L} n_{L}=n_{K} \ell_{\mu}^{L}+\ell_{\mathrm{id}}^{L}, \quad k_{L}=\ell_{\mu}^{L}+\ell_{\mathrm{id}}^{L} .
$$

Using (3.18) and (3.21), we obtain the $\ell$-shape as

$$
\ell_{\mu}^{L}=n_{L}-1, \quad \ell_{\mathrm{id}}^{L}=n_{K}-n_{L}
$$

Corollary 3.5. The arity $n_{L}$ of the semigroup $\mathcal{S}_{L}$ is less than or equal to the arity $n_{K}$ of the underlying polyadic field $n_{L} \leq n_{K}$.

3.2. Polyadic direct sum and tensor product. The Cartesian product of $D_{V}$ polyadic vector spaces

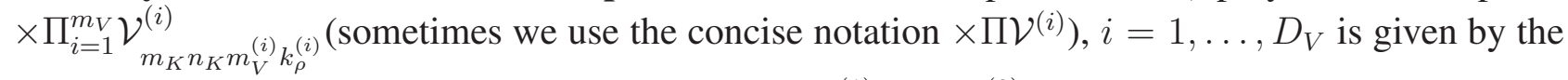
$D_{V}$-ples (an analog of the Cartesian pair $(\mathrm{v}, \mathrm{u}), \mathrm{v} \in \mathrm{V}^{(1)}, \mathrm{u} \in \mathrm{V}^{(2)}$ )

$$
\left(\begin{array}{c}
\mathbf{v}^{(1)} \\
\vdots \\
\mathbf{v}^{\left(D_{V}\right)}
\end{array}\right) \equiv\left(\mathbf{v}^{\left(D_{V}\right)}\right) \in \mathbf{V}^{\times D_{V}}
$$

We introduce a polyadic generalization of the direct sum and tensor product of vector spaces by considering "linear" operations on the $D_{V}$-ples (3.23).

In the first case, to endow $\times \Pi \mathcal{V}^{(i)}$ with the structure of a vector space we need to define a new operation between the $D_{V}$-ples (3.23) (similar to vector addition, but between elements from different spaces) and a rule, specifying how they are "multiplied by scalars" (analogs of $\left(\mathrm{v}_{1}, \mathrm{v}_{2}\right)+\left(\mathrm{u}_{1}, \mathrm{u}_{2}\right)=$ $\left(\mathrm{v}_{1}+\mathrm{u}_{1}, \mathrm{v}_{2}+\mathrm{u}_{2}\right)$ and $\left.\lambda\left(\mathrm{v}_{1}, \mathrm{v}_{2}\right)=\left(\lambda \mathrm{v}_{1}, \lambda \mathrm{v}_{2}\right)\right)$. In the binary case, a formal summation is used, but it can be different from the addition in the initial vector spaces. Therefore, we can define on the set of the $D_{V}$-ples (3.23) new operations $\chi_{m_{V}}$ ("addition of vectors from different spaces") and "multiplication by a scalar" $\tau_{k_{\rho}}$, which does not need to coincide with the corresponding operations $\nu_{m_{V}^{(i)}}^{(i)}$ and $\boldsymbol{\rho}_{k_{\rho}^{(i)}}^{(i)}$ of the initial polyadic vector spaces $\mathcal{V}_{m_{K}, n_{K}, m_{V}^{(i)}, k_{\rho}^{(i)}}^{(i)}$. 
If all $D_{V}$-ples (3.23) are of fixed length, then we can define their "addition" $\chi_{m_{V}}$ in the standard way, if all the arities $m_{V}^{(i)}$ coincide and equal the arity of the resulting vector space

$$
m_{V}=m_{V}^{(1)}=\ldots=m_{V}^{\left(D_{V}\right)}
$$

while the operations ("additions") themselves $\boldsymbol{\nu}_{m_{V}}^{(i)}$ between vectors in different spaces can be still different. Thus, a new commutative $m_{V}$-ary operation ("addition") $\chi_{m_{V}}$ of the $D_{V}$-ples of the same length is defined by

$$
\chi_{m_{V}}\left[\left(\begin{array}{c}
\mathrm{v}_{1}^{(1)} \\
\vdots \\
\mathrm{v}_{1}^{\left(D_{V}\right)}
\end{array}\right), \ldots,\left(\begin{array}{c}
\mathrm{v}_{m_{V}}^{(1)} \\
\vdots \\
\mathrm{v}_{m_{V}}^{\left(D_{V}\right)}
\end{array}\right)\right]=\left(\begin{array}{c}
\boldsymbol{\nu}_{m_{V}}^{(1)}\left[\mathrm{v}_{1}^{(1)}, \ldots, \mathrm{v}_{m_{V}}^{(1)}\right] \\
\vdots \\
\boldsymbol{\nu}_{m_{V}}^{\left(D_{V}\right)}\left[\mathrm{v}_{1}^{\left(D_{V}\right)}, \ldots, \mathrm{v}_{m_{V}}^{\left(D_{V}\right)}\right]
\end{array}\right)
$$

where $D_{V} \neq m_{V}$, in general. However, it is also possible to add $D_{V}$-ples of different length such that the operation (3.25) is compatible with all arities $m_{V}^{(i)}, i=1, \ldots, m_{V}$. For instance, if $m_{V}=3$, $m_{V}^{(1)}=m_{V}^{(2)}=3, m_{V}^{(3)}=2$, then

$$
\chi_{3}\left[\left(\begin{array}{c}
\mathrm{v}_{1}^{(1)} \\
\mathrm{v}_{1}^{(2)} \\
\mathrm{v}_{1}^{(3)}
\end{array}\right),\left(\begin{array}{c}
\mathrm{v}_{2}^{(1)} \\
\mathrm{v}_{2}^{(2)} \\
\mathrm{v}_{2}^{(3)}
\end{array}\right),\left(\begin{array}{c}
\mathrm{v}_{3}^{(1)} \\
\mathrm{v}_{3}^{(2)}
\end{array}\right)\right]=\left(\begin{array}{c}
\nu_{3}^{(1)}\left[\mathrm{v}_{1}^{(1)}, \mathrm{v}_{2}^{(1)}, \mathrm{v}_{3}^{(1)}\right] \\
\nu_{3}^{(2)}\left[\mathrm{v}_{1}^{(2)}, \mathrm{v}_{2}^{(2)}, \mathrm{v}_{3}^{(2)}\right] \\
\nu_{2}^{(3)}\left[\mathrm{v}_{1}^{(3)}, \mathrm{v}_{2}^{(3)}\right]
\end{array}\right) .
$$

Assertion 3.6. In the polyadic case, a direct sum of polyadic vector spaces having different arities of "vector addition" $m_{V}^{(i)}$ can be defined.

Let us introduce the multiaction $\tau_{k_{\rho}}$ ("multiplication by a scalar") acting on $D_{V}$-ple $\left(\mathbf{v}^{\left(m_{V}\right)}\right)$, then

$$
\left.\boldsymbol{\rho}_{k_{\rho}}\left\{\begin{array}{c}
\lambda_{1} \\
\vdots \\
\lambda_{k_{\rho}} \mid
\end{array} \mid\left(\begin{array}{c}
\mathbf{v}^{(1)} \\
\vdots \\
\mathbf{v}^{\left(D_{V}\right)}
\end{array}\right)\right\}=\left(\begin{array}{c}
\boldsymbol{\rho}_{k_{\rho}^{(1)}}^{(1)}\left\{\begin{array}{c}
\lambda_{1} \\
\vdots \\
\lambda_{k_{\rho}^{(1)}}
\end{array} \mid \mathbf{v}^{(1)}\right\} \\
\vdots \\
\boldsymbol{\rho}_{k_{\rho}^{\left(m_{V}\right)}}^{\left(D_{V}\right)} \\
\lambda_{k_{\rho}^{(1)}+\ldots+k_{\rho}^{\left(D_{V}\right)}} \mid
\end{array}\right\}\right)
$$

where

$$
k_{\rho}^{(1)}+\ldots+k_{\rho}^{\left(D_{V}\right)}=k_{\rho}
$$

Definition 3.7. A polyadic direct sum of $m_{V}$ polyadic vector spaces is their Cartesian product equipped with the $m_{V}$-ary addition $\chi_{m_{V}}$ and the $k_{\rho}$-place multiaction $\tau_{k_{\rho}}$, satisfying (3.25) and (3.27) respectively

$$
\oplus \Pi_{i=1}^{D_{V}} \mathcal{V}_{m_{K}, n_{K}, m_{V}^{(i)}, k_{\rho}^{(i)}}^{(i)}=\left\{\times \Pi_{i=1}^{D_{V}} \mathcal{V}_{m_{K}, n_{K}, m_{V}^{(i)}, k_{\rho}^{(i)}}^{(i)} \mid \chi_{m_{V}}, \boldsymbol{\tau}_{k_{\rho}}\right\}
$$

Let us consider another way to define a vector space structure on the $D_{V}$-ples from the Cartesian product $\times \Pi \mathcal{V}^{(i)}$. Remember that in the binary case, the concept of bilinearity is used, which means "distributivity" and "multiplicativity by scalars" on each place separately in the Cartesian pair 
$\left(\mathrm{v}_{1}, \mathrm{v}_{2}\right) \in \mathrm{V}^{(1)} \times \mathrm{V}^{(2)}$ (as opposed to the direct sum, where these relations hold on all places simultaneously, see (3.25) and (3.27) such that

$$
\begin{aligned}
\left(\mathrm{v}_{1}+\mathrm{u}_{1}, \mathrm{v}_{2}\right) & =\left(\mathrm{v}_{1}, \mathrm{v}_{2}\right)+\left(\mathrm{u}_{1}, \mathrm{v}_{2}\right), \quad\left(\mathrm{v}_{1}, \mathrm{v}_{2}+\mathrm{u}_{2}\right)=\left(\mathrm{v}_{1}, \mathrm{v}_{2}\right)+\left(\mathrm{v}_{1}, \mathrm{u}_{2}\right), \\
\lambda\left(\mathrm{v}_{1}, \mathrm{v}_{2}\right) & =\left(\lambda \mathrm{v}_{1}, \mathrm{v}_{2}\right)=\left(\mathrm{v}_{1}, \lambda \mathrm{v}_{2}\right),
\end{aligned}
$$

respectively. If we denote the ideal corresponding to the relations (3.30)-3.31) by $\mathrm{B}_{2}$, then the binary tensor product of the vector spaces can be defined as their Cartesian product by factoring out this ideal, as $\mathcal{V}^{(1)} \otimes \mathcal{V}^{(2)} \stackrel{\text { def }}{=} \mathcal{V}^{(1)} \times \mathcal{V}^{(2)} / \mathrm{B}_{2}$. Note first, that the additions and multiplications by a scalar on both sides of (3.30)-(3.31) "work" in different spaces, which sometimes can be concealed by adding the word "formal" to them. Second, all these operations have the same arity (binary ones), which need not be the case when considering polyadic structures.

As in the case of the polyadic direct sum, we first define a new operation $\tilde{\chi}_{m_{V}}$ ("addition") of the $D_{V}$-ples of fixed length (different from $\chi_{m_{V}}$ in (3.25)), when all the arities $m_{V}^{(i)}$ coincide and equal to $m_{V}$ (3.24). Then, a straightforward generalization of (3.30) can be defined for $m_{V}$-ples similar to the polyadic distributivity (1.1)-(1.3), as in the following $m_{V}$ relations

$$
\begin{aligned}
& \left(\begin{array}{c}
\boldsymbol{\nu}_{m_{V}}^{(1)}\left[\mathrm{v}_{1}^{(1)}, \ldots, \mathrm{v}_{m_{V}}^{(1)}\right] \\
\mathrm{u}_{2} \\
\vdots \\
\mathrm{u}_{D_{V}}
\end{array}\right)=\tilde{\boldsymbol{\chi}}_{m_{V}}\left[\left(\begin{array}{c}
\mathrm{v}_{1}^{(1)} \\
\mathrm{u}_{2} \\
\vdots \\
\mathrm{u}_{D_{V}}
\end{array}\right), \ldots,\left(\begin{array}{c}
\mathrm{v}_{m_{V}}^{(1)} \\
\mathrm{u}_{2} \\
\vdots \\
\mathrm{u}_{D_{V}}
\end{array}\right)\right] \text {, } \\
& \left(\begin{array}{c}
\boldsymbol{\nu}_{m_{V}}^{(2)}\left[\mathrm{v}_{1}^{(2)}, \ldots, \mathrm{v}_{m_{V}}^{(2)}\right] \\
\vdots \\
\mathrm{u}_{m_{V}}
\end{array}\right)=\tilde{\boldsymbol{\chi}}_{m_{V}}\left[\left(\begin{array}{c}
\mathrm{u}_{1} \\
\mathrm{v}_{1}^{(2)} \\
\vdots \\
\mathrm{u}_{m_{V}}
\end{array}\right), \ldots,\left(\begin{array}{c}
\mathrm{u}_{1} \\
\mathrm{v}_{m_{V}}^{(2)} \\
\vdots \\
\mathrm{u}_{m_{V}}
\end{array}\right)\right] \\
& \left(\begin{array}{c}
\mathrm{u}_{1} \\
\mathrm{u}_{2} \\
\vdots \\
\boldsymbol{\nu}_{m_{V}}^{\left(m_{V}\right)}\left[\mathrm{v}_{1}^{\left(D_{V}\right)}, \ldots, \mathrm{v}_{m_{V}}^{\left(D_{V}\right)}\right]
\end{array}\right)=\tilde{\boldsymbol{\chi}}_{m_{V}}\left[\left(\begin{array}{c}
\mathrm{u}_{1} \\
\mathrm{u}_{2} \\
\vdots \\
\mathrm{v}_{1}^{\left(D_{V}\right)}
\end{array}\right), \ldots,\left(\begin{array}{c}
\mathrm{u}_{1} \\
\mathrm{u}_{2} \\
\vdots \\
\mathrm{v}_{m_{V}}^{\left(D_{V}\right)}
\end{array}\right)\right]
\end{aligned}
$$

By analogy, if all $k_{\rho}^{(i)}$ are equal we can define a new multiaction $\tilde{\tau}_{k_{\rho}}$ (different from $\tau_{k_{\rho}}$ (3.27) ) with the same number of places

$$
k_{\rho}=k_{\rho}^{(1)}=\ldots=k_{\rho}^{\left(D_{V}\right)}
$$

as the $D_{V}$ relations (an analog of (3.31))

$$
\boldsymbol{\rho}_{k_{\rho}}^{\prime}\left\{\begin{array}{c}
\lambda_{1} \\
\vdots \\
\lambda_{k_{\rho}}
\end{array} \mid\left(\begin{array}{c}
\mathbf{v}^{(1)} \\
\vdots \\
\mathbf{v}^{\left(D_{V}\right)}
\end{array}\right)\right\}=\left(\begin{array}{c}
\boldsymbol{\rho}_{k_{\rho}}^{(1)}\left\{\begin{array}{c}
\lambda_{1} \\
\vdots \\
\lambda_{k_{\rho}}
\end{array} \mid \mathbf{v}^{(1)}\right\} \\
\mathrm{v}^{(2)} \\
\vdots \\
\mathbf{v}^{\left(D_{V}\right)}
\end{array}\right)
$$




$$
\begin{aligned}
& =\left(\begin{array}{c}
\boldsymbol{v}^{(1)} \\
\left.\boldsymbol{\rho}_{k_{\rho}}^{(2)}\left\{\begin{array}{c}
\lambda_{1} \\
\vdots \\
\lambda_{k_{\rho}}
\end{array}\right) \mathbf{v}^{(2)}\right\} \\
\vdots \\
\mathbf{v}^{\left(D_{V}\right)}
\end{array}\right) \\
& \left.=\left(\begin{array}{c}
\mathbf{v}^{(1)} \\
\mathbf{v}^{(2)} \\
\vdots \\
\lambda_{1} \\
\vdots \\
\lambda_{k_{\rho}}
\end{array}\right\} \mathbf{v}^{\left(D_{V}\right)}\right\}
\end{aligned}
$$

Let us denote the ideal corresponding to the relations (3.32)-3.34), (3.36-3.38) by $\mathrm{B}_{D_{V}}$.

Definition 3.8. A polyadic tensor product of $D_{V}$ polyadic vector spaces $\mathcal{V}_{m_{K}, n_{K}, m_{V}^{(i)}, k_{\rho}^{(i)}}^{(i)}$ is their Cartesian product equipped with the $m_{V}$-ary addition $\tilde{\chi}_{m_{V}}$ (of $D_{V}$-ples) and the $k_{\rho}$-place multiaction $\tilde{\tau}_{k_{\rho}}$, satisfying (3.32)-3.34) and (3.36)-3.38), respectively, by factoring out the ideal $\mathrm{B}_{D_{V}}$

$$
\otimes \Pi_{i=1}^{m_{V}} \mathcal{V}_{m_{K}, n_{K}, m_{V}^{(i)}, k_{\rho}^{(i)}}^{(i)}=\left\{\times \Pi_{i=1}^{m_{V}} \mathcal{V}_{m_{K}, n_{K}, m_{V}^{(i)}, k_{\rho}^{(i)}}^{(i)} \mid \tilde{\boldsymbol{\chi}}_{m_{V}}, \tilde{\boldsymbol{\tau}}_{k_{\rho}}\right\} / \mathrm{B}_{D_{V}}
$$

As in the case of the polyadic direct sum, we can consider the distributivity for $D_{V}$-ples of different length. In a similar example (3.26), if $m_{V}=3, m_{V}^{(1)}=m_{V}^{(2)}=3, m_{V}^{(3)}=2$, we have

$$
\begin{aligned}
\left(\begin{array}{c}
\boldsymbol{\nu}_{3}^{(1)}\left[\mathrm{v}_{1}^{(1)}, \mathrm{v}_{2}^{(1)}, \mathrm{v}_{3}^{(1)}\right] \\
\mathrm{u}_{2} \\
\mathrm{u}_{3}
\end{array}\right)=\tilde{\boldsymbol{\chi}}_{3}\left[\left(\begin{array}{c}
\mathrm{v}_{1}^{(1)} \\
\mathrm{u}_{2} \\
\mathrm{u}_{3}
\end{array}\right),\left(\begin{array}{c}
\mathrm{v}_{2}^{(1)} \\
\mathrm{u}_{2} \\
\mathrm{u}_{3}
\end{array}\right),\left(\begin{array}{c}
\mathrm{v}_{3}^{(1)} \\
\mathrm{u}_{2}
\end{array}\right)\right], \\
\left(\begin{array}{c}
\mathrm{u}_{1} \\
\boldsymbol{\nu}_{3}^{(2)}\left[\mathrm{v}_{1}^{(2)}, \mathrm{v}_{2}^{(2)}, \mathrm{v}_{3}^{(2)}\right] \\
\mathrm{u}_{3}
\end{array}\right)=\tilde{\chi}_{3}\left[\left(\begin{array}{c}
\mathrm{u}_{1} \\
\mathrm{v}_{1}^{(2)} \\
\mathrm{u}_{3}
\end{array}\right),\left(\begin{array}{c}
\mathrm{u}_{1} \\
\mathrm{v}_{2}^{(2)} \\
\mathrm{u}_{3}
\end{array}\right),\left(\begin{array}{c}
\mathrm{u}_{1} \\
\mathrm{v}_{3}^{(2)}
\end{array}\right)\right], \\
\left(\begin{array}{c}
\mathrm{u}_{1} \\
\mathrm{u}_{2} \\
\boldsymbol{\nu}_{2}^{(3)}\left[\mathrm{v}_{1}^{(3)}, \mathrm{v}_{2}^{(3)}\right]
\end{array}\right)=\tilde{\chi}_{3}\left[\left(\begin{array}{c}
\mathrm{u}_{1} \\
\mathrm{u}_{2} \\
\mathrm{v}_{1}^{(3)}
\end{array}\right),\left(\begin{array}{c}
\mathrm{u}_{1} \\
\mathrm{u}_{2} \\
\mathrm{v}_{2}^{(3)}
\end{array}\right),\left(\begin{array}{l}
\mathrm{u}_{1} \\
\mathrm{u}_{2}
\end{array}\right)\right] .
\end{aligned}
$$

Assertion 3.9. A tensor product of polyadic vector spaces having different arities of the "vector addition" $m_{V}^{(i)}$ can be defined.

In the case of modules over a polyadic ring, the formulas connecting arities and $\ell$-shapes similar to those above hold, while concrete properties (noncommutativity, mediality, etc.) should be taken into account. 


\section{POLYADIC INNER PAIRING SPACES AND NORMS}

Here we introduce the next important operation: a polyadic analog of the inner product for polyadic vector spaces - a polyadic inner pairing. Let $\mathcal{V}_{m_{K}, n_{K}, m_{V}, k_{\rho}}=\left\langle K ; \mathrm{V}\left|\boldsymbol{\sigma}_{m_{K}}, \boldsymbol{\kappa}_{n_{K}} ; \boldsymbol{\nu}_{m_{V}}\right| \boldsymbol{\rho}_{k_{\rho}}\right\rangle$ be a polyadic vector space over the polyadic field $\mathbb{K}_{m_{K}, n_{K}}$ (2.22). By analogy with the binary inner product, we introduce its polyadic counterpart and study its arity shape.

Definition 4.1. A polyadic $N$-place inner pairing (an analog of the inner product) is a mapping

$$
\overbrace{\langle\langle\bullet|\bullet| \ldots \mid \bullet\rangle\rangle}^{N}: \mathrm{V}^{\times N} \rightarrow K
$$

satisfying the following conditions:

1) Polyadic "linearity" (2.9) (for first argument):

$$
\left\langle\left\langle\boldsymbol{\rho}_{k_{\rho}}\left\{\begin{array}{c}
\lambda_{1} \\
\vdots \\
\lambda_{k_{\rho}}
\end{array} \mid \mathrm{v}_{1}\right\}\left|\mathrm{v}_{2}\right| \ldots \mid \mathrm{v}_{N}\right\rangle\right\rangle=\boldsymbol{\kappa}_{n_{K}}\left[\lambda_{1}, \ldots, \lambda_{k_{\rho}},\left\langle\left\langle\mathrm{v}_{1}\left|\mathrm{v}_{2}\right| \ldots \mid \mathrm{v}_{N}\right\rangle\right\rangle\right] .
$$

2) Polyadic "distributivity" (1.1)-(1.3) (on each place):

$$
\begin{aligned}
& \left\langle\left\langle\boldsymbol{\nu}_{m_{V}}\left[\mathrm{v}_{1}, \mathrm{u}_{1}, \ldots \mathrm{u}_{m_{V}-1}\right]\left|\mathrm{v}_{2}\right| \ldots \mid \mathrm{v}_{N}\right\rangle\right\rangle \\
& =\boldsymbol{\sigma}_{m_{K}}\left[\left\langle\left\langle\mathrm{v}_{1}\left|\mathrm{v}_{2}\right| \ldots \mid \mathrm{v}_{N}\right\rangle\right\rangle,\left\langle\left\langle\mathrm{u}_{1}\left|\mathrm{v}_{2}\right| \ldots \mid \mathrm{v}_{N}\right\rangle\right\rangle \ldots\left\langle\left\langle\mathrm{u}_{m_{V}-1}\left|\mathrm{v}_{2}\right| \ldots \mid \mathrm{v}_{N}\right\rangle\right\rangle\right] .
\end{aligned}
$$

If the polyadic field $\mathbb{K}_{m_{K}, n_{K}}$ contains the zero $z_{K}$ and $\left\langle\mathrm{V} \mid m_{V}\right\rangle$ has the zero "vector" $z_{V}$ (which is not always the case in the polyadic case), we have the additional axiom:

3) The polyadic inner pairing vanishes $\left\langle\left\langle\mathrm{v}_{1}\left|\mathrm{v}_{2}\right| \ldots \mid \mathrm{v}_{N}\right\rangle\right\rangle=z_{K}$, iff any of the "vectors" vanishes, $\exists i \in 1, \ldots, N$, such that $\mathrm{v}_{i}=\mathrm{z}_{V}$.

If the standard binary ordering on $\mathbb{K}_{m_{K}, n_{K}}$ can be defined, then the polyadic inner pairing satisfies:

4) The positivity condition

$$
\overbrace{\langle\langle\mathrm{v}|\mathrm{v}| \ldots \mid \mathrm{v}\rangle\rangle}^{N} \geq z_{K}
$$

5) The polyadic Cauchy-Schwarz inequality ("triangle" inequality)

$$
\begin{gathered}
\boldsymbol{\kappa}_{n_{K}}[\overbrace{\left.\langle\overbrace{\left\langle\left\langle\mathrm{v}_{1}\left|\mathrm{v}_{1}\right| \ldots \mid \mathrm{v}_{1}\right\rangle\right\rangle}^{N}, \overbrace{\left\langle\left\langle\mathrm{v}_{2}\left|\mathrm{v}_{2}\right| \ldots \mid \mathrm{v}_{2}\right\rangle\right\rangle}^{N} \ldots \overbrace{\left\langle\left\langle\mathrm{v}_{n_{K}}\right| \mathrm{v}_{\left.n_{K}|\ldots| \mathrm{v}_{n_{K}}\right\rangle}\right.}^{n_{K}}]\right\rangle}^{N}] \\
\geq \boldsymbol{\kappa}_{n_{K}}[\overbrace{\left\langle\left\langle\mathrm{v}_{1}\left|\mathrm{v}_{2}\right| \ldots \mid \mathrm{v}_{N}\right\rangle\right\rangle,\left\langle\left\langle\mathrm{v}_{1}\left|\mathrm{v}_{2}\right| \ldots \mid \mathrm{v}_{N}\right\rangle\right\rangle \ldots,\left\langle\left\langle\mathrm{v}_{1}\left|\mathrm{v}_{2}\right| \ldots \mid \mathrm{v}_{N}\right\rangle\right\rangle}^{n_{K}}] .
\end{gathered}
$$

To make the above relations consistent, the arity shapes should be fixed.

Definition 4.2. If the inner pairing is fully symmetric under permutations it is called a polyadic inner product.

Proposition 4.3. The number of places in the multiaction $\rho_{k_{\rho}}$ differs by 1 from the multiplication arity of the polyadic field

$$
n_{K}-k_{\rho}=1
$$

Proof. It follows from the polyadic "linearity" (4.2).

\footnotetext{
${ }^{2}$ Note that this concept is different from the $n$-inner product spaces considered in [Misiak, et al].
} 
Proposition 4.4. The arities of "vector addition" and "field addition" coincide

$$
m_{V}=m_{K}
$$

Proof. Implied by the polyadic "distributivity" (4.3).

Proposition 4.5. The arity of the "field multiplication" is equal to the arity of the polyadic inner pairing space

$$
n_{K}=N .
$$

Proof. This follows from the polyadic Cauchy-Schwarz inequality (4.5).

Definition 4.6. The polyadic vector space $\mathcal{V}_{m_{K}, n_{K}, m_{V}, k_{\rho}}$ equipped with the polyadic inner pairing $\overbrace{\langle\bullet \bullet \bullet|\ldots| \bullet\rangle\rangle}^{N}: \mathrm{V}^{\times N} \rightarrow K$ is called a polyadic inner pairing space $\mathcal{H}_{m_{K}, n_{K}, m_{V}, k_{\rho}, N}$.

A polyadic analog of the binary norm $\|\bullet\|: \mathrm{V} \rightarrow K$ can be induced by the inner pairing similarly to the binary case for the inner product (we use the form $\|v\|^{2}=\langle\langle v \mid v\rangle\rangle$ ).

Definition 4.7. A polyadic norm of a "vector" $v$ in the polyadic inner pairing space $\mathcal{H}_{m_{K}, n_{K}, m_{V}, k_{\rho}, N}$ is a mapping $\|\bullet\|_{N}: \mathrm{V} \rightarrow K$, such that

$$
\begin{aligned}
\boldsymbol{\kappa}_{n_{K}}[\overbrace{\|\mathrm{v}\|_{N},\|\mathrm{v}\|_{N}, \ldots,\|\mathrm{v}\|_{N}}^{n_{K}}] & =\overbrace{\langle\langle\mathrm{v}|\mathrm{v}| \ldots \mid \mathrm{v}\rangle\rangle}^{N}, \\
n_{K} & =N,
\end{aligned}
$$

and the following axioms apply:

1) The polyadic "linearity"

$$
\begin{aligned}
& \left\|\boldsymbol{\rho}_{k_{\rho}}\left\{\begin{array}{c}
\lambda_{1} \\
\vdots \\
\lambda_{k_{\rho}}
\end{array} \mid \mathrm{v}\right\}\right\|_{N}=\boldsymbol{\kappa}_{n_{K}}\left[\lambda_{1}, \ldots, \lambda_{k_{\rho}},\|\mathrm{v}\|_{N}\right], \\
& n_{K}-k_{\rho}=1 .
\end{aligned}
$$

If the polyadic field $\mathbb{K}_{m_{K}, n_{K}}$ contains the zero $z_{K}$ and $\left\langle\mathrm{V} \mid m_{V}\right\rangle$ has a zero "vector" $z_{V}$, then:

2) The polyadic norm vanishes $\|\mathrm{v}\|_{N}=z_{K}$, iff $\mathrm{v}=\mathrm{z}_{V}$.

If the binary ordering on $\left\langle\mathrm{V} \mid m_{V}\right\rangle$ can be defined, then:

3) The polyadic norm is positive $\|\mathrm{v}\|_{N} \geq z_{K}$.

4) The polyadic "triangle" inequality holds

$$
\begin{aligned}
\boldsymbol{\sigma}_{m_{K}}[\overbrace{\left\|\mathrm{v}_{1}\right\|_{N},\left\|\mathrm{v}_{2}\right\|_{N}, \ldots,\left\|\mathrm{v}_{N}\right\|_{N}}^{m_{K}}] \geq\left\|\boldsymbol{\nu}_{m_{V}}[\overbrace{\left\|\mathrm{v}_{1}\right\|_{N},\left\|\mathrm{v}_{2}\right\|_{N}, \ldots,\left\|\mathrm{v}_{N}\right\|_{N}}^{m_{V}}]\right\|, \\
m_{K}=m_{V}=N .
\end{aligned}
$$

Definition 4.8. The polyadic inner pairing space $\mathcal{H}_{m_{K}, n_{K}, m_{V}, k_{\rho}, N}$ equipped with the polyadic norm $\|\mathrm{v}\|_{N}$ is called a polyadic normed space.

Recall that in the binary vector space $\mathrm{V}$ over the field $\mathbb{K}$ equipped with the inner product $\langle\langle\bullet \mid \bullet\rangle\rangle$ and the norm $\|\bullet\|$, one can introduce the angle between vectors $\left\|\mathrm{v}_{1}\right\| \cdot\left\|\mathrm{v}_{2}\right\| \cdot \cos \theta=\left\langle\left\langle\mathrm{v}_{1} \mid \mathrm{v}_{2}\right\rangle\right\rangle$, where on 1.h.s. there are two binary multiplications $(\cdot)$.

Definition 4.9. A polyadic angle between $N$ vectors $\mathrm{v}_{1}, \mathrm{v}_{2}, \ldots, \mathrm{v}_{n_{K}}$ of the polyadic inner pairing space $\mathcal{H}_{m_{K}, n_{K}, m_{V}, k_{\rho}, N}$ is defined as a set of angles $\boldsymbol{\vartheta}=\left\{\left\{\theta_{i}\right\} \mid i=1,2, \ldots, n_{K}-1\right\}$ satisfying

$$
\boldsymbol{\kappa}_{n_{K}}^{(2)}\left[\left\|\mathrm{v}_{1}\right\|_{N},\left\|\mathrm{v}_{2}\right\|_{N}, \ldots,\left\|\mathrm{v}_{n_{K}}\right\|_{N}, \cos \theta_{1}, \cos \theta_{2}, \ldots, \cos \theta_{n_{K}-1}\right]=\left\langle\left\langle\mathrm{v}_{1}\left|\mathrm{v}_{2}\right| \ldots \mid \mathrm{v}_{n_{K}}\right\rangle\right\rangle,
$$


where $\boldsymbol{\kappa}_{n_{K}}^{(2)}$ is a long product of two $n_{K}$-ary multiplications, which consists of $2\left(n_{K}-1\right)+1$ terms.

We will not consider the completion with respect to the above norm (to obtain a polyadic analog of Hilbert space) and corresponding limits and boundedness questions, because it will not give additional arity shapes, in which we are mostly interested here. Instead, below we turn to some applications and new general constructions which appear from the above polyadic structures.

TABLE 2. The arity signature and arity shape of polyadic algebraic structures.

\begin{tabular}{|c|c|c|c|c|c|c|c|c|c|}
\hline \multirow{2}{*}{ Structures } & \multicolumn{2}{|c|}{ Sets } & \multicolumn{6}{|c|}{ Operations and arities } & \multirow{2}{*}{$\begin{array}{l}\text { Arity } \\
\text { shape }\end{array}$} \\
\hline & $\mathrm{N}$ & Name & $\mathrm{N}$ & \multicolumn{2}{|c|}{ Multiplications } & \multicolumn{2}{|c|}{ Additions } & Multiactions & \\
\hline \multicolumn{10}{|c|}{ Group-like polyadic algebraic structures } \\
\hline $\begin{array}{l}n \text {-ary magma } \\
\text { (or groupoid) }\end{array}$ & 1 & $M$ & 1 & \multicolumn{2}{|c|}{$\begin{array}{c}\mu_{n}: \\
M^{n} \rightarrow M\end{array}$} & & & & \\
\hline $\begin{array}{l}n \text {-ary semigroup } \\
\text { (and monoid) }\end{array}$ & 1 & $S$ & 1 & \multicolumn{2}{|c|}{$\begin{array}{c}\mu_{n}: \\
S^{n} \rightarrow S\end{array}$} & & & & \\
\hline $\begin{array}{l}n \text {-ary quasigroup } \\
\text { (and loop) }\end{array}$ & 1 & $Q$ & 1 & \multicolumn{2}{|c|}{$\begin{array}{c}\mu_{n}: \\
Q^{n} \rightarrow Q\end{array}$} & & & & \\
\hline \multicolumn{10}{|c|}{ Ring-like polyadic algebraic structures } \\
\hline$(m, n)$-ary ring & 1 & $R$ & 2 & \multicolumn{2}{|c|}{$\begin{array}{c}\mu_{n}: \\
R^{n} \rightarrow R\end{array}$} & \multicolumn{2}{|c|}{$\begin{array}{c}\nu_{m}: \\
R^{m} \rightarrow R\end{array}$} & & \\
\hline$(m, n)$-ary field & 1 & $K$ & 2 & \multicolumn{2}{|c|}{$\begin{array}{c}\mu_{n}: \\
K^{n} \rightarrow K\end{array}$} & \multicolumn{2}{|c|}{$\begin{array}{c}\nu_{m}: \\
K^{m} \rightarrow K\end{array}$} & & \\
\hline $\begin{array}{c}\text { Vector space } \\
\text { over }\left(m_{K}, n_{K}\right) \text {-field }\end{array}$ & 2 & $K, \mathrm{~V}$ & 4 & \multicolumn{2}{|c|}{$\begin{array}{c}\sigma_{n_{K}}: \\
K^{n_{K} \rightarrow K}\end{array}$} & $\begin{array}{c}\kappa_{m_{K}}: \\
K^{m_{K} \rightarrow K}\end{array}$ & $\begin{array}{c}\nu_{m_{V}}: \\
\mathrm{V}^{m_{V} \rightarrow \mathrm{V}}\end{array}$ & $\begin{array}{c}\rho_{k_{\rho}}: \\
K^{k_{\rho}} \times \mathrm{V} \rightarrow \mathrm{V}\end{array}$ & $\begin{array}{l}2.14 \\
2.21 \\
\end{array}$ \\
\hline \multicolumn{10}{|c|}{ Algebra-like polyadic algebraic structures } \\
\hline $\begin{array}{l}\text { Inner pairing space } \\
\text { over }\left(m_{K}, n_{K}\right) \text {-field }\end{array}$ & 2 & $K, \mathrm{~V}$ & 5 & $\begin{array}{c}\sigma_{n_{K}}: \\
K^{n_{K}} \rightarrow K \\
\end{array}$ & $\begin{array}{c}N \text {-Form } \\
\langle\langle\bullet . . \bullet\rangle\rangle: \\
\mathrm{V}^{N} \rightarrow K\end{array}$ & $\begin{array}{c}\kappa_{m_{K}}: \\
K^{m_{K} \rightarrow K}\end{array}$ & $\begin{array}{c}\nu_{m_{V}}: \\
\mathrm{V}^{m_{V}} \rightarrow \mathrm{V}\end{array}$ & $\begin{array}{c}\rho_{k_{\rho}}: \\
K^{k_{\rho}} \times \mathrm{V} \rightarrow \mathrm{V}\end{array}$ & $\begin{array}{l}4.6 \\
4.7 \\
4.8 \\
4\end{array}$ \\
\hline $\begin{array}{c}\left(m_{A}, n_{A}\right) \text {-algebra } \\
\text { over }\left(m_{K}, n_{K}\right) \text {-field }\end{array}$ & 2 & $K, \mathrm{~A}$ & 5 & $\begin{array}{c}\sigma_{n_{K}}: \\
K^{n_{K} \rightarrow K}\end{array}$ & $\begin{array}{c}\mu_{n_{A}}: \\
\mathrm{A}^{n} \rightarrow \mathrm{A}\end{array}$ & $\begin{array}{c}\kappa_{m_{K}}: \\
K^{m_{K} \rightarrow K}\end{array}$ & $\begin{array}{c}\nu_{m_{A}}: \\
\mathrm{A}^{m_{M}} \rightarrow \mathrm{A}\end{array}$ & $\begin{array}{c}\rho_{k_{\rho}}: \\
K^{k_{\rho} \times \mathrm{A}} \rightarrow \mathrm{A}\end{array}$ & 2.35 \\
\hline
\end{tabular}

To conclude, we present the resulting TABLE 2 in which the polyadic algebraic structures are listed together with their arity shapes.

\section{Applications}

\section{ELEMENTS OF POLYADIC OPERATOR THEORY}

Here we consider the 1-place polyadic operators $\boldsymbol{T}=\boldsymbol{F}_{k_{F}=1}$ (the case $k_{F}=1$ of the mapping $\boldsymbol{F}_{k_{F}}$ in Definition 3.2) on polyadic inner pairing spaces and structurally generalize the adjointness and involution concepts.

Remark 5.1. A polyadic operator is a complicated mapping between polyadic vector spaces having nontrivial arity shape (3.4) which is actually an action on a set of "vectors". However, only for $k_{F}=1$ it can be written in a formal way multiplicatively, as it is always done in the binary case.

Recall (to fix notations and observe analogies) the informal standard introduction of the operator algebra and the adjoint operator on a binary pre-Hilbert space $\mathcal{H}\left(\equiv \mathcal{H}_{m_{K}=2, n_{K}=2, m_{V}=2, k_{\rho}=1, N=2}\right)$ over a binary field $\mathbb{K}\left(\equiv \mathbb{K}_{m_{K}=2, n_{K}=2}\right.$ ) (having the underlying set $\{K ; \bigvee\}$ ). For the operator norm $\|\bullet\|_{T}:\{\boldsymbol{T}\} \rightarrow K$ we use (among many others) the following definition

$$
\|\boldsymbol{T}\|_{T}=\inf \{M \in K \mid\|\boldsymbol{T} \vee\| \leq M\|\mathrm{v}\|, \forall \mathrm{v} \in \mathrm{V}\},
$$


which is convenient for further polyadic generalization. Bounded operators have $M<\infty$. If on the set of operators $\{\boldsymbol{T}\}$ (as 1-place mappings $\mathrm{V} \rightarrow \mathrm{V}$ ) one defines the addition $\left(+_{T}\right)$, product $\left(\circ_{T}\right)$ and scalar multiplication $\left(\cdot_{T}\right)$ in the standard way

$$
\begin{aligned}
\left(\boldsymbol{T}_{1}+_{T} \boldsymbol{T}_{2}\right)(\mathrm{v}) & =\boldsymbol{T}_{1} \mathrm{v}+\boldsymbol{T}_{2} \mathrm{v}, \\
\left(\boldsymbol{T}_{1} \circ_{T} \boldsymbol{T}_{2}\right)(\mathrm{v}) & =\boldsymbol{T}_{1}\left(\boldsymbol{T}_{2} \mathrm{v}\right), \\
\left(\lambda \bullet_{T} \boldsymbol{T}\right)(\mathrm{v}) & =\lambda(\boldsymbol{T} \mathrm{v}), \quad \lambda \in K, \quad \mathrm{v} \in \mathrm{V},
\end{aligned}
$$

then $\left\langle\{\boldsymbol{T}\}\left|+_{T}, \circ_{T}\right| \cdot_{T}\right\rangle$ becomes an operator algebra $\mathcal{A}_{T}$ (associativity and distributivity are obvious). The unity $\boldsymbol{I}$ and zero $Z$ of $\mathcal{A}_{T}$ (if they exist), satisfy

$$
\begin{aligned}
& \boldsymbol{I} \mathrm{v}=\mathrm{v}, \\
& Z \mathrm{v}=\mathrm{z}_{V}, \quad \forall \mathrm{v} \in \mathrm{V},
\end{aligned}
$$

respectively, where $z_{V} \in \mathrm{V}$ is the polyadic "zero-vector".

The connection between operators, linear functionals and inner products is given by the Riesz representation theorem. Informally, it states that in a binary pre-Hilbert space $\mathcal{H}=\{K ; \mathrm{V}\}$ a (bounded) linear functional (sesquilinear form) $\boldsymbol{L}: \mathrm{V} \times \mathrm{V} \rightarrow K$ can be uniquely represented as

$$
\boldsymbol{L}\left(\mathrm{v}_{1}, \mathrm{v}_{2}\right)=\left\langle\left\langle\boldsymbol{T} \mathrm{v}_{1} \mid \mathrm{v}_{2}\right\rangle\right\rangle_{\text {sym }}, \quad \forall \mathrm{v}_{1}, \mathrm{v}_{2} \in \mathrm{V},
$$

where $\langle\langle\bullet \mid \bullet\rangle\rangle_{\text {sym }}: \mathrm{V} \times \mathrm{V} \rightarrow K$ is a (binary) inner product with standard properties and $\boldsymbol{T}: \mathrm{V} \rightarrow \mathrm{V}$ is a bounded linear operator, such that the norms of $\boldsymbol{L}$ and $\boldsymbol{T}$ coincide. Because the linear functionals form a dual space (see Subsection 3.1), the relation (5.7) fixes the shape of its elements. The main consequence of the Riesz representation theorem is the existence of the adjoint: for any (bounded) linear operator $T: \bigvee \rightarrow \bigvee$ there exists a (unique bounded) adjoint operator $\boldsymbol{T}^{*}: \mathrm{V} \rightarrow \mathrm{V}$ satisfying

$$
\boldsymbol{L}\left(\mathrm{v}_{1}, \mathrm{v}_{2}\right)=\left\langle\left\langle\boldsymbol{T} \mathrm{v}_{1} \mid \mathrm{v}_{2}\right\rangle\right\rangle_{\text {sym }}=\left\langle\left\langle\mathrm{v}_{1} \mid \boldsymbol{T}^{*} \mathrm{v}_{2}\right\rangle\right\rangle_{\text {sym }}, \quad \forall \mathrm{v}_{1}, \mathrm{v}_{2} \in \mathrm{V},
$$

and the norms of $\boldsymbol{T}$ and $\boldsymbol{T}^{*}$ are equal. It follows from the conjugation symmetry of the standard binary inner product, that (5.8) coincides with

$$
\left\langle\left\langle\mathrm{v}_{1} \mid \boldsymbol{T} \mathrm{v}_{2}\right\rangle\right\rangle_{\text {sym }}=\left\langle\left\langle\boldsymbol{T}^{*} \mathrm{v}_{1} \mid \mathrm{v}_{2}\right\rangle\right\rangle_{\text {sym }}, \quad \forall \mathrm{v}_{1}, \mathrm{v}_{2} \in \mathrm{V} .
$$

However, when $\langle\langle\bullet \mid \bullet\rangle\rangle$ has no symmetry (permutation, conjugation, etc., see, e.g. MIGNOT [1976]), it becomes the binary $(N=2)$ inner pairing (4.1), the binary adjoint consists of 2 operators $\left(\boldsymbol{T}^{\star_{12}}\right) \neq\left(\boldsymbol{T}^{\star_{21}}\right), \boldsymbol{T}^{\star_{i j}}: \mathrm{V} \rightarrow \mathrm{V}$, which should be defined by 2 equations

$$
\begin{aligned}
& \left\langle\left\langle T \mathrm{v}_{1} \mid \mathrm{v}_{2}\right\rangle\right\rangle=\left\langle\left\langle\mathrm{v}_{1} \mid T^{\star 12} \mathrm{v}_{2}\right\rangle\right\rangle, \\
& \left\langle\left\langle\mathrm{v}_{1} \mid T \mathrm{v}_{2}\right\rangle\right\rangle=\left\langle\left\langle\boldsymbol{T}^{\star 21} \mathrm{v}_{1} \mid \mathrm{v}_{2}\right\rangle\right\rangle,
\end{aligned}
$$

where $\left(\star_{12}\right) \neq\left(\star_{21}\right)$ are 2 different star operations satisfying 2 relations

$$
\begin{aligned}
& \boldsymbol{T}^{\star_{12 \star_{21}}}=\boldsymbol{T}, \\
& \boldsymbol{T}^{\star_{21} \star_{12}}=\boldsymbol{T} .
\end{aligned}
$$

If $\langle\langle\bullet \mid \bullet\rangle\rangle=\langle\langle\bullet \mid \bullet\rangle\rangle_{\text {sym }}$ is symmetric, it becomes the inner product in the pre-Hilbert space $\mathcal{H}$ and the equations (5.12) $-(5.13)$ coincide, while the operation $(*)=\left(\star_{12}\right)=\left(\star_{21}\right)$ stands for the standard involution

$$
T^{* *}=T .
$$


5.1. Multistars and polyadic adjoints. Consider now a special case of the polyadic inner pairing space (see Definition 4.6)

$$
\mathcal{H}_{m_{K}, n_{K}, m_{V}, k_{\rho}=1, N}=\langle K ; \mathrm{V}\left|\boldsymbol{\sigma}_{m_{K}}, \boldsymbol{\kappa}_{n_{K}} ; \boldsymbol{\nu}_{m_{V}}\right| \boldsymbol{\rho}_{k_{\rho}=1} \mid \overbrace{\langle\langle\bullet|\ldots| \bullet\rangle\rangle}^{N}\rangle
$$

with 1-place multiaction $\boldsymbol{\rho}_{k_{\rho}=1}$.

Definition 5.2. The set of 1-place operators $\boldsymbol{T}: \mathrm{V} \rightarrow \mathrm{V}$ together with the set of "scalars" $K$ become a polyadic operator algebra $\mathcal{A}_{T}=\left\langle K ;\{\boldsymbol{T}\}\left|\boldsymbol{\sigma}_{m_{K}}, \boldsymbol{\kappa}_{n_{K}} ; \boldsymbol{\eta}_{m_{T}}, \boldsymbol{\omega}_{n_{T}}\right| \boldsymbol{\theta}_{k_{F}=1}\right\rangle$, if the operations $\boldsymbol{\eta}_{m_{T}}, \boldsymbol{\omega}_{n_{T}}, \boldsymbol{\theta}_{k_{F}=1}$ to define by

$$
\begin{aligned}
\boldsymbol{\eta}_{m_{T}}\left[\boldsymbol{T}_{1}, \boldsymbol{T}_{2}, \ldots, \boldsymbol{T}_{m_{T}}\right](\mathrm{v}) & =\boldsymbol{\nu}_{m_{V}}\left[\boldsymbol{T}_{1} \mathrm{v}, \boldsymbol{T}_{2} \mathrm{v}, \ldots, \boldsymbol{T}_{m_{T}} \mathrm{v}\right], \\
\boldsymbol{\omega}_{n_{T}}\left[\boldsymbol{T}_{1}, \boldsymbol{T}_{2}, \ldots, \boldsymbol{T}_{n_{T}}\right](\mathrm{v}) & =\boldsymbol{T}_{1}\left(\boldsymbol{T}_{2} \ldots\left(\boldsymbol{T}_{n_{T}} \mathrm{v}\right)\right), \\
\boldsymbol{\theta}_{k_{F}=1}\{\lambda \mid \boldsymbol{T}\}(\mathrm{v}) & =\boldsymbol{\rho}_{k_{\rho}=1}\{\lambda \mid \boldsymbol{T} \mathrm{v}\}, \forall \lambda \in K, \forall \mathrm{v} \in \mathrm{V} .
\end{aligned}
$$

The arity shape is fixed by

Proposition 5.3. In the polyadic algebra $\mathcal{A}_{T}$ the arity of the operator addition $m_{T}$ coincides with the "vector" addition of the inner pairing space $m_{V}$, i.e.

$$
m_{T}=m_{V}
$$

Proof. This follows from (5.16).

To get relations between operators we assume (as in the binary case) uniqueness: for any $\boldsymbol{T}_{1}, \boldsymbol{T}_{2}$ : $\mathrm{V} \rightarrow \mathrm{V}$ it follows from

$$
\left\langle\left\langle\mathrm{v}_{1}\left|\mathrm{v}_{2}\right| \ldots\left|\boldsymbol{T}_{1} \mathrm{v}_{i}\right| \ldots \mathrm{v}_{N-1} \mid \mathrm{v}_{N}\right\rangle\right\rangle=\left\langle\left\langle\mathrm{v}_{1}\left|\mathrm{v}_{2}\right| \ldots\left|\boldsymbol{T}_{2} \mathrm{v}_{i}\right| \ldots \mathrm{v}_{N-1} \mid \mathrm{v}_{N}\right\rangle\right\rangle,
$$

that $\boldsymbol{T}_{1}=\boldsymbol{T}_{2}$ on any place $i=1, \ldots, N$.

First, by analogy with the binary adjoint (5.8) we define $N$ different adjoints for each operator $\boldsymbol{T}$.

Definition 5.4. Given a polyadic operator $\boldsymbol{T}: \mathrm{V} \rightarrow \mathrm{V}$ on the polyadic inner pairing space $\mathcal{H}_{m_{K}, n_{K}, m_{V}, k_{\rho}=1, N}$ we define a polyadic adjoint as the set $\left\{\boldsymbol{T}^{\star_{i j}}\right\}$ of $N$ operators $\boldsymbol{T}^{\star_{i j}}$ satisfying the following $N$ equations

$$
\begin{aligned}
& \left\langle\left\langle\boldsymbol{T} \mathrm{v}_{1}\left|\mathrm{v}_{2}\right| \mathrm{v}_{3}|\ldots| \mathrm{v}_{N}\right\rangle\right\rangle=\left\langle\left\langle\mathrm{v}_{1}\left|\boldsymbol{T}^{\star_{12}} \mathrm{v}_{2}\right| \mathrm{v}_{3}|\ldots| \mathrm{v}_{N}\right\rangle\right\rangle, \\
& \left\langle\left\langle\mathrm{v}_{1}\left|\boldsymbol{T} \mathrm{v}_{2}\right| \mathrm{v}_{3}|\ldots| \mathrm{v}_{N}\right\rangle\right\rangle=\left\langle\left\langle\mathrm{v}_{1}\left|\mathrm{v}_{2}\right| \boldsymbol{T}^{\star_{23}} \mathrm{v}_{3}|\ldots| \mathrm{v}_{N}\right\rangle\right\rangle, \\
& \vdots \\
& \left\langle\left\langle\mathrm{v}_{1}\left|\mathrm{v}_{2}\right| \mathrm{v}_{3}\left|\ldots \boldsymbol{T} \mathrm{v}_{N-1}\right| \mathrm{v}_{N}\right\rangle\right\rangle=\left\langle\left\langle\mathrm{v}_{1}\left|\mathrm{v}_{2}\right| \mathrm{v}_{3}|\ldots| \boldsymbol{T}^{\star_{N-1, N}} \mathrm{v}_{N}\right\rangle\right\rangle, \\
& \left\langle\left\langle\mathrm{v}_{1}\left|\mathrm{v}_{2}\right| \mathrm{v}_{3}\left|\ldots \mathrm{v}_{N-1}\right| \boldsymbol{T} \mathrm{v}_{N}\right\rangle\right\rangle=\left\langle\left\langle\boldsymbol{T}^{\star_{N}, 1} \mathrm{v}_{1}\left|\mathrm{v}_{2}\right| \mathrm{v}_{3}|\ldots| \mathrm{v}_{N}\right\rangle\right\rangle, \quad \mathrm{v}_{i} \in \mathrm{v} .
\end{aligned}
$$

In what follows, for the composition we will use the notation $\left(\boldsymbol{T}^{\star_{i j}}\right)^{\star k l \cdots} \equiv \boldsymbol{T}^{\star_{i j} \star_{k l} \cdots}$. We have from (5.21) the $N$ relations

$$
\begin{gathered}
\boldsymbol{T}^{\star_{12} \star_{23} \star_{34} \ldots \star_{N-1, N} \star_{N, 1}}=\boldsymbol{T}, \\
\boldsymbol{T}^{\star_{23} \star_{34} \ldots \star_{N-1, N{ }^{\star}, 1 \star_{12}}}=\boldsymbol{T}, \\
\vdots \\
\boldsymbol{T}^{\star_{N, 1} \star_{12} \star_{23} \star_{34} \cdots \star_{N-1, N}}=\boldsymbol{T},
\end{gathered}
$$

which are called multistar cycles.

Definition 5.5. We call the set of adjoint mappings $\left(\bullet^{\star_{i j}}\right): \boldsymbol{T} \rightarrow \boldsymbol{T}^{\star_{i j}}$ a polyadic involution, if they satisfy the multistar cycles (5.22). 
If the inner pairing $\langle\langle\bullet|\ldots| \bullet\rangle\rangle$ has more than two places $N \geq 3$, we have some additional structural issues, which do not exist in the binary case.

First, we observe that the set of the adjointness relations (5.21) can be described in the framework of the associativity quiver approach introduced in DUPLIJ [2012] for polyadic representations. That is, for general $N \geq 3$ in addition to (5.21) which corresponds to the so called Post-like associativity quiver (they will be called the Post-like adjointness relations), there also exist other sets. It is cumbersome to write additional general formulas like (5.21) for other non-Post-like cases, while instead we give a clear example for $N=4$.

Example 5.6. The polyadic adjointness relations for $N=4$ consist of the sets corresponding to different associativity quivers

$$
\begin{array}{cc}
\text { 1) Post-like adjointness relations } & \text { 2) Non-Post-like adjointness relations } \\
\left\langle\left\langle\boldsymbol{T} \mathrm{v}_{1}\left|\mathrm{v}_{2}\right| \mathrm{v}_{3} \mid \mathrm{v}_{4}\right\rangle\right\rangle=\left\langle\left\langle\mathrm{v}_{1}\left|\boldsymbol{T}^{\star 12} \mathrm{v}_{2}\right| \mathrm{v}_{3} \mid \mathrm{v}_{4}\right\rangle\right\rangle, & \left\langle\left\langle\boldsymbol{T} \mathrm{v}_{1}\left|\mathrm{v}_{2}\right| \mathrm{v}_{3} \mid \mathrm{v}_{4}\right\rangle\right\rangle=\left\langle\left\langle\mathrm{v}_{1}\left|\mathrm{v}_{2}\right| \mathrm{v}_{3} \mid \boldsymbol{T}^{\star 14} \mathrm{v}_{4}\right\rangle\right\rangle, \\
\left\langle\left\langle\mathrm{v}_{1}\left|\boldsymbol{T} \mathrm{v}_{2}\right| \mathrm{v}_{3} \mid \mathrm{v}_{4}\right\rangle\right\rangle=\left\langle\left\langle\mathrm{v}_{1}\left|\mathrm{v}_{2}\right| \boldsymbol{T}^{\star 23} \mathrm{v}_{3} \mid \mathrm{v}_{4}\right\rangle\right\rangle, & \left\langle\left\langle\mathrm{v}_{1}\left|\mathrm{v}_{2}\right| \mathrm{v}_{3} \mid \boldsymbol{T} \mathrm{v}_{4}\right\rangle\right\rangle=\left\langle\left\langle\mathrm{v}_{1}\left|\mathrm{v}_{2}\right| \boldsymbol{T}^{\star 43} \mathrm{v}_{3} \mid \mathrm{v}_{4}\right\rangle\right\rangle, \\
\left\langle\left\langle\mathrm{v}_{1}\left|\mathrm{v}_{2}\right| \boldsymbol{T} \mathrm{v}_{3} \mid \mathrm{v}_{4}\right\rangle\right\rangle=\left\langle\left\langle\mathrm{v}_{1}\left|\mathrm{v}_{2}\right| \mathrm{v}_{3} \mid \boldsymbol{T}^{\star 34} \mathrm{v}_{4}\right\rangle\right\rangle, & \left\langle\left\langle\mathrm{v}_{1}\left|\mathrm{v}_{2}\right| \boldsymbol{T} \mathrm{v}_{3} \mid \mathrm{v}_{4}\right\rangle\right\rangle=\left\langle\left\langle\mathrm{v}_{1}\left|\boldsymbol{T}^{\star 32} \mathrm{v}_{2}\right| \mathrm{v}_{3} \mid \mathrm{v}_{4}\right\rangle\right\rangle, \\
\left\langle\left\langle\mathrm{v}_{1}\left|\mathrm{v}_{2}\right| \mathrm{v}_{3} \mid \boldsymbol{T} \mathrm{v}_{4}\right\rangle\right\rangle=\left\langle\left\langle\boldsymbol{T}^{\star 41} \mathrm{v}_{1}\left|\mathrm{v}_{2}\right| \mathrm{v}_{3} \mid \mathrm{v}_{4}\right\rangle\right\rangle, & \left\langle\left\langle\mathrm{v}_{1}\left|\boldsymbol{T} \mathrm{v}_{2}\right| \mathrm{v}_{3} \mid \mathrm{v}_{4}\right\rangle\right\rangle=\left\langle\left\langle\boldsymbol{T}^{\star 21} \mathrm{v}_{1}\left|\mathrm{v}_{2}\right| \mathrm{v}_{3} \mid \mathrm{v}_{4}\right\rangle\right\rangle,
\end{array}
$$

and the corresponding multistar cycles

$$
\begin{aligned}
& \text { 1) Post-like multistar cycles } \\
& \boldsymbol{T}^{\star_{12} \star_{23} \star_{34} \star_{41}}=\boldsymbol{T}, \\
& \boldsymbol{T}^{\star_{23} \star_{34} \star_{41} \star_{12}}=\boldsymbol{T}, \\
& \boldsymbol{T}^{\star_{34} \star_{41} \star_{12} \star_{23}}=\boldsymbol{T}, \\
& \boldsymbol{T}^{\star_{41} \star_{12} \star_{23} \star_{34}}=\boldsymbol{T},
\end{aligned}
$$

2) Non-Post-like multistar cycles

$$
\begin{aligned}
& \boldsymbol{T}^{\star_{14} \star_{43} \star_{32} \star_{21}}=\boldsymbol{T}, \\
& \boldsymbol{T}^{\star_{43} \star_{22} \star_{21}{ }^{\star} 4}=\boldsymbol{T}, \\
& \boldsymbol{T}^{\star_{32} \star_{21} \star_{14} \star_{43}}=\boldsymbol{T}, \\
& \boldsymbol{T}^{\star_{21} \star_{14} \star^{\star} \star_{32}}=\boldsymbol{T} .
\end{aligned}
$$

Thus, if the inner pairing has no symmetry, then both the Post-like and non-Post-like adjoints and corresponding multistar involutions are different.

Second, in the case $N \geq 3$ any symmetry of the multiplace inner pairing restricts the polyadic adjoint sets and multistar involutions considerably.

Theorem 5.7. If the inner pairing with $N \geq 3$ has the full permutation symmetry

$$
\left\langle\left\langle\mathrm{v}_{1}\left|\mathrm{v}_{2}\right| \ldots \mid \mathrm{v}_{N}\right\rangle\right\rangle=\left\langle\left\langle\sigma \mathrm{v}_{1}\left|\sigma \mathrm{v}_{2}\right| \ldots \mid \sigma \mathrm{v}_{N}\right\rangle\right\rangle, \quad \forall \sigma \in \mathrm{S}_{N}
$$

where $\mathrm{S}_{N}$ is the symmetric group of $N$ elements, then:

1) All the multistars coincide $\left(\star_{i j}\right)=\left(\star_{k l}\right):=(*)$ for any allowed $i, j, k, l=1, \ldots, N$;

2) All the operators are self-adjoint $\boldsymbol{T}=\boldsymbol{T}^{*}$.

\section{Proof.}

1) In each adjointness relation from (5.21) we place the operator $T$ on the 1.h.s. to the first position and its multistar adjoint $\boldsymbol{T}^{\star_{i j}}$ to the second position, using the full permutation symmetry, which together with (5.20) gives the equality of all multistar operations.

2) We place the operator $\boldsymbol{T}$ on the 1.h.s. to the first position and apply the derivation of the involution in the binary case to increasing cycles of size $i \leq N$ recursively, that is:

For $i=2$

$$
\begin{aligned}
\left\langle\left\langle\boldsymbol{T} \mathrm{v}_{1}\left|\mathrm{v}_{2}\right| \mathrm{v}_{3}|\ldots| \mathrm{v}_{N}\right\rangle\right\rangle & =\left\langle\left\langle\mathrm{v}_{1}\left|\boldsymbol{T}^{*} \mathrm{v}_{2}\right| \mathrm{v}_{3}|\ldots| \mathrm{v}_{N}\right\rangle\right\rangle=\left\langle\left\langle\boldsymbol{T}^{*} \mathrm{v}_{2}\left|\mathrm{v}_{1}\right| \mathrm{v}_{3}|\ldots| \mathrm{v}_{N}\right\rangle\right\rangle \\
& =\left\langle\left\langle\mathrm{v}_{2}\left|\boldsymbol{T}^{* *} \mathrm{v}_{1}\right| \mathrm{v}_{3}|\ldots| \mathrm{v}_{N}\right\rangle\right\rangle=\left\langle\left\langle\boldsymbol{T}^{* *} \mathrm{v}_{1}\left|\mathrm{v}_{2}\right| \mathrm{v}_{3}|\ldots| \mathrm{v}_{N}\right\rangle\right\rangle
\end{aligned}
$$

then, using (5.20) we get

$$
\boldsymbol{T}=\boldsymbol{T}^{* *}
$$

as in the standard binary case. However, for $N \geq 3$ we have $N$ higher cycles in addition. 
For $i=3$

$$
\begin{aligned}
\left\langle\left\langle\boldsymbol{T} \mathrm{v}_{1}\left|\mathrm{v}_{2}\right| \mathrm{v}_{3}|\ldots| \mathrm{v}_{N}\right\rangle\right\rangle & =\left\langle\left\langle\mathrm{v}_{1}\left|\boldsymbol{T}^{*} \mathrm{v}_{2}\right| \mathrm{v}_{3}|\ldots| \mathrm{v}_{N}\right\rangle\right\rangle=\left\langle\left\langle\boldsymbol{T}^{*} \mathrm{v}_{2}\left|\mathrm{v}_{3}\right| \mathrm{v}_{1}|\ldots| \mathrm{v}_{N}\right\rangle\right\rangle \\
& =\left\langle\left\langle\mathrm{v}_{2}\left|\boldsymbol{T}^{* *} \mathrm{v}_{3}\right| \mathrm{v}_{1}|\ldots| \mathrm{v}_{N}\right\rangle\right\rangle=\left\langle\left\langle\boldsymbol{T}^{* *} \mathrm{v}_{3}\left|\mathrm{v}_{1}\right| \mathrm{v}_{2}|\ldots| \mathrm{v}_{N}\right\rangle\right\rangle \\
& =\left\langle\left\langle\mathrm{v}_{3}\left|\boldsymbol{T}^{* * *} \mathrm{v}_{1}\right| \mathrm{v}_{2}|\ldots| \mathrm{v}_{N}\right\rangle\right\rangle=\left\langle\left\langle\boldsymbol{T}^{* * *} \mathrm{v}_{1}\left|\mathrm{v}_{2}\right| \mathrm{v}_{3}|\ldots| \mathrm{v}_{N}\right\rangle\right\rangle,
\end{aligned}
$$

which together with (5.20) gives

$$
\boldsymbol{T}=\boldsymbol{T}^{* * *},
$$

and after using (5.27)

$$
T=T^{*}
$$

Similarly, for an arbitrary length of the cycle $i$ we obtain $T=T^{* * \ldots *}$, which should be valid for each cycle recursively with $i=2,3, \ldots, N$. Therefore, for any $N \geq 3$ all the operators $\boldsymbol{T}$ are self-adjoint (5.30), while $N=2$ is an exceptional case, when we have $\boldsymbol{T}=$ $T^{* *}$ (5.27) only.

Now we show that imposing a partial symmetry on the polyadic inner pairing will give more interesting properties to the adjoint operators. Recall, that one of possible binary commutativity generalizations of (semi)groups to the polyadic case is the semicommutativity concept, when in the multiplication only the first and last elements are exchanged. Similarly, we introduce

Definition 5.8. The polyadic inner pairing is called semicommutative, if

$$
\left\langle\left\langle\mathrm{v}_{1}\left|\mathrm{v}_{2}\right| \mathrm{v}_{3}|\ldots| \mathrm{v}_{N}\right\rangle\right\rangle=\left\langle\left\langle\mathrm{v}_{N}\left|\mathrm{v}_{2}\right| \mathrm{v}_{3}|\ldots| \mathrm{v}_{1}\right\rangle\right\rangle, \quad \mathrm{v}_{i} \in \mathrm{V} .
$$

Proposition 5.9. If the polyadic inner pairing is semicommutative, then for any operator $\boldsymbol{T}$ (satisfying Post-like adjointness (5.21)) the last multistar operation $\left(\star_{N, 1}\right)$ is a binary involution and is a composition of all the previous multistars

$$
\begin{aligned}
\boldsymbol{T}^{\star_{N}, 1} & =\boldsymbol{T}^{\star_{12} \star_{23} \star_{34} \cdots \star_{N-1, N}}, \\
\boldsymbol{T}^{\star_{N, 1} \star_{N, 1}} & =\boldsymbol{T} .
\end{aligned}
$$

Proof. It follows from (5.21) and (5.31), that

$$
\begin{aligned}
\left\langle\left\langle\mathrm{v}_{1}\left|\mathrm{v}_{2}\right| \mathrm{v}_{3}|\ldots| \boldsymbol{T} \mathrm{v}_{N}\right\rangle\right\rangle & =\left\langle\left\langle\boldsymbol{T} \mathrm{v}_{N}\left|\mathrm{v}_{2}\right| \mathrm{v}_{3}|\ldots| \mathrm{v}_{1}\right\rangle\right\rangle=\left\langle\left\langle\mathrm{v}_{N}\left|\mathrm{v}_{2}\right| \mathrm{v}_{3}|\ldots| \boldsymbol{T}^{\star_{12} \star_{23} \star_{34} \ldots \star_{N-1, N}} \mathrm{v}_{1}\right\rangle\right\rangle \\
& =\left\langle\left\langle\boldsymbol{T}^{\star_{12} \star_{23} \star_{34} \ldots \star_{N-1, N}} \mathrm{v}_{1}\left|\mathrm{v}_{2}\right| \mathrm{v}_{3}|\ldots| \mathrm{v}_{N}\right\rangle\right\rangle=\left\langle\left\langle\boldsymbol{T}^{\star_{N}, 1} \mathrm{v}_{1}\left|\mathrm{v}_{2}\right| \mathrm{v}_{3}|\ldots| \mathrm{v}_{N}\right\rangle\right\rangle,
\end{aligned}
$$

which using (5.20) gives (5.32), (5.33) follows from the first multistar cocycle in (5.22).

The adjointness relations (5.21) (of all kinds) together with (5.18) and (5.19) allows us to fix the arity shape of the polyadic operator algebra $\mathcal{A}_{T}$. We will assume that the arity of the operator multiplication in $\mathcal{A}_{T}$ coincides with the number of places of the inner pairing $N$ (4.1)

$$
n_{T}=N
$$

because it is in agreement with (5.21). Thus, the arity shape of the polyadic operator algebra becomes

$$
\mathcal{A}_{T}=\left\langle K ;\{\boldsymbol{T}\}\left|\boldsymbol{\sigma}_{m_{K}}, \boldsymbol{\kappa}_{n_{K}} ; \boldsymbol{\eta}_{m_{T}=m_{V}}, \boldsymbol{\omega}_{n_{T}=N}\right| \boldsymbol{\theta}_{k_{F}=k_{\rho}=1}\right\rangle,
$$

Definition 5.10. We call the operator algebra $\mathcal{A}_{T}$ which has the arity $n_{T}=N$ a nonderived polyadic operator algebra.

Let us investigate some structural properties of $\mathcal{A}_{T}$ and types of polyadic operators. 
Remark 5.11. We can only define, but not derive, as in the binary case, the action of any multistar $\left(\star_{i j}\right)$ on the product of operators, because in the nonderived $n_{T}$-ary algebra we have a fixed number of operators in a product and sum, that is $\ell^{\prime}\left(n_{T}-1\right)+1$ and $\ell^{\prime \prime}\left(m_{T}-1\right)+1$, correspondingly, where $\ell^{\prime}$ is the number of $n_{T}$-ary multiplications and $\ell^{\prime}$ is the number of $m_{T}$-ary additions. Therefore, we cannot transfer (one at a time) all the polyadic operators from one place in the inner pairing to another place, as is done in the standard proof in the binary case.

Taking this into account, as well as consistency under the multistar cycles (5.22), we arrive at

Definition 5.12. The fixed multistar operation acts on the $\ell=1$ product of $n_{T}$ polyadic operators, depending on the sequential number of the multistar $\left(\star_{i j}\right)$ (for the Post-like adjointness relations (5.21)

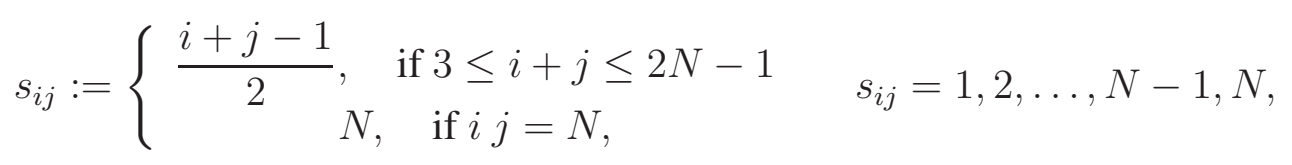

in the following way

$$
\left(\boldsymbol{\omega}_{n_{T}}\left[\boldsymbol{T}_{1}, \boldsymbol{T}_{2}, \ldots, \boldsymbol{T}_{n_{T}-1}, \boldsymbol{T}_{n_{T}}\right]\right)^{\star_{i j}}=\left\{\begin{array}{cl}
\boldsymbol{\omega}_{n_{T}}\left[\boldsymbol{T}_{n_{T}}^{\star_{i j}}, \boldsymbol{T}_{n_{T}-1}^{\star_{i j}}, \ldots, \boldsymbol{T}_{2}^{\star_{i j}}, \boldsymbol{T}_{1}^{\star_{i j}}\right], & \text { if } s_{i j} \text { is odd, } \\
\boldsymbol{\omega}_{n_{T}}\left[\boldsymbol{T}_{1}^{\star_{i j}}, \boldsymbol{T}_{2}^{\star_{i j}}, \ldots, \boldsymbol{T}_{n_{T}-1}^{\star_{i j}}, \boldsymbol{T}_{n_{T}}^{\star_{i j}}\right], & \text { if } s_{i j} \text { is even. }
\end{array}\right.
$$

A rule similar to (5.39) holds also for non-Post-like adjointness relations, but their concrete form depends of the corresponding non-Post-like associative quiver.

Sometimes, to shorten notation, it will be more convenient to mark a multistar by the sequential number (5.38), such that $\left(\star_{i j}\right) \Rightarrow\left(\star_{s_{i j}}\right)$, e.g. $\left(\star_{23}\right) \Rightarrow\left(\star_{2}\right),\left(\star_{N, 1}\right) \Rightarrow\left(\star_{N}\right)$, etc. Also, in examples, for the ternary multiplication we will use the square brackets without the name of operation, if it is clear from the context, e.g. $\boldsymbol{\omega}_{3}\left[\boldsymbol{T}_{1}, \boldsymbol{T}_{2}, \boldsymbol{T}_{3}\right] \Rightarrow\left[\boldsymbol{T}_{1}, \boldsymbol{T}_{2}, \boldsymbol{T}_{3}\right]$, etc.

Example 5.13. In the lowest ternary case $N=3$ we have

$$
\begin{array}{ll}
\text { 1) Post-like adjointness relations } & \text { 2) Non-Post-like adjointness relations } \\
\left\langle\left\langle\boldsymbol{T} \mathrm{v}_{1}\left|\mathrm{v}_{2}\right| \mathrm{v}_{3}\right\rangle\right\rangle=\left\langle\left\langle\mathrm{v}_{1}\left|\boldsymbol{T}^{\star_{1}} \mathrm{v}_{2}\right| \mathrm{v}_{3}\right\rangle\right\rangle, & \left\langle\left\langle\boldsymbol{T} \mathrm{v}_{1}\left|\mathrm{v}_{2}\right| \mathrm{v}_{3}\right\rangle\right\rangle=\left\langle\left\langle\mathrm{v}_{1}\left|\mathrm{v}_{2}\right| \boldsymbol{T}^{\star_{3}} \mathrm{v}_{3}\right\rangle\right\rangle, \\
\left\langle\left\langle\mathrm{v}_{1}\left|\boldsymbol{T} \mathrm{v}_{2}\right| \mathrm{v}_{3}\right\rangle\right\rangle=\left\langle\left\langle\mathrm{v}_{1}\left|\mathrm{v}_{2}\right| \boldsymbol{T}^{\star 2} \mathrm{v}_{3}\right\rangle\right\rangle, & \left\langle\left\langle\mathrm{v}_{1}\left|\mathrm{v}_{2}\right| \boldsymbol{T} \mathrm{v}_{3}\right\rangle\right\rangle=\left\langle\left\langle\mathrm{v}_{1}\left|\boldsymbol{T}^{\star_{2}} \mathrm{v}_{2}\right| \mathrm{v}_{3}\right\rangle\right\rangle, \\
\left\langle\left\langle\mathrm{v}_{1}\left|\mathrm{v}_{2}\right| \boldsymbol{T} \mathrm{v}_{3}\right\rangle\right\rangle=\left\langle\left\langle\boldsymbol{T}^{\star_{3}} \mathrm{v}_{1}\left|\mathrm{v}_{2}\right| \mathrm{v}_{3}\right\rangle\right\rangle, & \left\langle\left\langle\mathrm{v}_{1}\left|\boldsymbol{T} \mathrm{v}_{2}\right| \mathrm{v}_{3}\right\rangle\right\rangle=\left\langle\left\langle\boldsymbol{T}^{\star_{1}} \mathrm{v}_{1}\left|\mathrm{v}_{2}\right| \mathrm{v}_{3}\right\rangle\right\rangle,
\end{array}
$$

and the corresponding multistar cycles

$$
\begin{array}{rc}
\text { 1) Post-like multistar cycles } & \text { 2) Non-Post-like multistar cycles } \\
\boldsymbol{T}^{\star_{1} \star_{2} \star_{3}}=\boldsymbol{T}, & \boldsymbol{T}^{\star_{3} \star_{2} \star_{1}}=\boldsymbol{T}, \\
\boldsymbol{T}^{\star_{2} \star_{3} \star_{1}}=\boldsymbol{T}, & \boldsymbol{T}^{\star_{2} \star_{1} \star_{3}}=\boldsymbol{T}, \\
\boldsymbol{T}^{\star_{3} \star_{1} \star_{2}}=\boldsymbol{T}, & \boldsymbol{T}^{\star_{1} \star_{3} \star_{2}}=\boldsymbol{T} .
\end{array}
$$

Using (5.39) we obtain the following ternary conjugation rules

$$
\begin{aligned}
& \left(\left[\boldsymbol{T}_{1}, \boldsymbol{T}_{2}, \boldsymbol{T}_{3}\right]\right)^{\star 1}=\left[\boldsymbol{T}_{3}^{\star 1}, \boldsymbol{T}_{2}^{\star 1}, \boldsymbol{T}_{1}^{\star 1}\right], \\
& \left(\left[\boldsymbol{T}_{1}, \boldsymbol{T}_{2}, \boldsymbol{T}_{3}\right]\right)^{\star_{2}}=\left[\boldsymbol{T}_{1}^{\star_{2}}, \boldsymbol{T}_{2}^{\star_{2}}, \boldsymbol{T}_{3}^{\star_{2}}\right], \\
& \left(\left[\boldsymbol{T}_{1}, \boldsymbol{T}_{2}, \boldsymbol{T}_{3}\right]\right)^{\star_{3}}=\left[\boldsymbol{T}_{3}^{\star_{3}}, \boldsymbol{T}_{2}^{\star_{3}}, \boldsymbol{T}_{1}^{\star 3}\right],
\end{aligned}
$$

which are common for both Post-like and non-Post-like adjointness relations (5.40).

Definition 5.14. A polyadic operator $\boldsymbol{T}$ is called self-adjoint, if all multistar operations are identities, i.e. $\left(\star_{i j}\right)=\mathrm{id}, \forall i, j$. 
5.2. Polyadic isometry and projection. Now we introduce polyadic analogs for the important types of operators: isometry, unitary, and (orthogonal) projection. Taking into account Remark 5.11, we again cannot move operators singly, and instead of proving the operator relations, as it is usually done in the binary case, we can only exploit some mnemonic rules to define the corresponding relations between polyadic operators.

If the polyadic operator algebra $\mathcal{A}_{T}$ contains a unit $I$ and zero $Z$ (see (5.5)-(5.6) ) we define the conditions of polyadic isometry and orthogonality:

Definition 5.15. A polyadic operator $T$ is called a polyadic isometry, if it preserves the polyadic inner pairing

$$
\left\langle\left\langle\boldsymbol{T} \mathrm{v}_{1}\left|\boldsymbol{T} \mathrm{v}_{2}\right| \boldsymbol{T} \mathrm{v}_{3}|\ldots| \boldsymbol{T} \mathrm{v}_{N}\right\rangle\right\rangle=\left\langle\left\langle\mathrm{v}_{1}\left|\vee_{2}\right| \vee_{3}|\ldots| \vee_{N}\right\rangle\right\rangle
$$

and satisfies

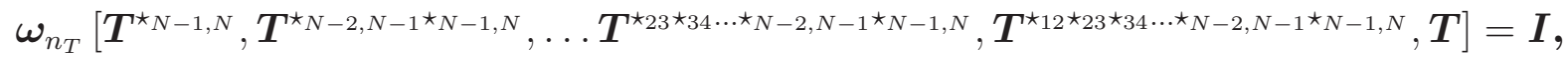

$$
\begin{aligned}
& +(N-1) \text { cycle permutations of multistars in the first }(N-1) \text { terms. }
\end{aligned}
$$

Remark 5.16. If the multiplication in $\mathcal{A}_{T}$ is derived and all multistars are equal, then the polyadic isometry operators satisfy some kind of $N$-regularity DUPLIJ AND MARCINEK [2002] or regular $N$-cocycle condition DUPLIJ AND MARCINEK [2001].

Proposition 5.17. The polyadic isometry operator $\boldsymbol{T}$ preserves the polyadic norm

$$
\|\boldsymbol{T} \mathrm{v}\|_{N}=\|\mathrm{v}\|_{N}, \quad \forall \mathrm{v} \in \mathrm{V} .
$$

Proof. It follows from (4.9) and (5.45), that

$$
\boldsymbol{\kappa}_{n_{K}}[\overbrace{\|\boldsymbol{T} \mathrm{v}\|_{N},\|\boldsymbol{T} \mathrm{v}\|_{N}, \ldots,\|\boldsymbol{T} \mathrm{v}\|_{N}}^{n_{K}}]=\boldsymbol{\kappa}_{n_{K}}[\overbrace{\|\mathrm{v}\|_{N},\|\mathrm{v}\|_{N}, \ldots,\|\mathrm{v}\|_{N}}^{n_{K}}],
$$

which gives (5.47), when $n_{K}=N$.

Definition 5.18. If for $N$ polyadic operators $\boldsymbol{T}_{i}$ we have

$$
\left\langle\left\langle\boldsymbol{T}_{1} \mathrm{v}_{1}\left|\boldsymbol{T}_{2} \mathrm{v}_{2}\right| \boldsymbol{T}_{3} \mathrm{v}_{3}|\ldots| \boldsymbol{T}_{N} \mathrm{v}_{N}\right\rangle\right\rangle=\mathrm{z}_{K}, \quad \forall \mathrm{v}_{i} \in \mathrm{V},
$$

where $\mathrm{z}_{K} \in \mathrm{V}$ is the zero of the underlying polyadic field $\mathbb{K}_{m_{K}, n_{K}}$, then we say that $\boldsymbol{T}_{i}$ are (polyadically) orthogonal, and they satisfy

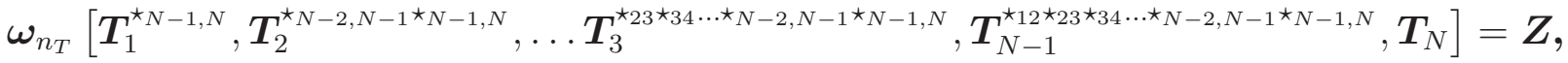

$$
\begin{aligned}
& +(N-1) \text { cycle permutations of multistars in the first }(N-1) \text { terms. }
\end{aligned}
$$

The polyadic analog of projection is given by

Definition 5.19. If a polyadic operator $\boldsymbol{P} \in \mathcal{A}_{T}$ satisfies the polyadic idempotency condition

$$
\boldsymbol{\omega}_{n_{T}}[\overbrace{\boldsymbol{P}, \boldsymbol{P}, \ldots \boldsymbol{P}}^{n_{T}}]=\boldsymbol{P}
$$

then it is called a polyadic projection.

By analogy with the binary case, polyadic projections can be constructed from polyadic isometry operators in a natural way. 
Proposition 5.20. If $\boldsymbol{T} \in \mathcal{A}_{T}$ is a polyadic isometry, then

$$
\begin{aligned}
\boldsymbol{P}_{\boldsymbol{T}}^{(1)} & =\boldsymbol{\omega}_{n_{T}}\left[\boldsymbol{T}, \boldsymbol{T}^{\star_{N-1, N}}, \boldsymbol{T}^{\star_{N-2, N-1} \star_{N-1, N}}, \ldots \boldsymbol{T}^{\star_{23} \star_{34} \ldots \star_{N-2, N-1} \star_{N-1, N}}, \boldsymbol{T}^{\star_{12} \star_{23} \star_{34} \ldots \star_{N-2, N-1} \star_{N-1, N}}\right], \\
& +(N-1) \text { cycle permutations of multistars in the last }(N-1) \text { terms. }
\end{aligned}
$$

are the corresponding polyadic projections $\boldsymbol{P}_{\boldsymbol{T}}^{(k)}, k=1, \ldots, N$, satisfying (5.52).

Definition 5.21. A polyadic operator $\boldsymbol{T} \in \mathcal{A}_{T}$ is called normal, if

$$
\begin{aligned}
& \boldsymbol{\omega}_{n_{T}}\left[\boldsymbol{T}^{\star_{N-1, N}}, \boldsymbol{T}^{\star_{N-2, N-1} \star_{N-1, N}}, \ldots \boldsymbol{T}^{\star_{23} \star_{34} \ldots \star_{N-2, N-1} \star_{N-1, N}}, \boldsymbol{T}^{\star_{12} \star_{23} \star_{34} \ldots \star_{N-2, N-1} \star_{N-1, N}}, \boldsymbol{T}\right]= \\
& \boldsymbol{\omega}_{n_{T}}\left[\boldsymbol{T}, \boldsymbol{T}^{\star_{N-1, N}}, \boldsymbol{T}^{\star_{N-2, N-1} \star_{N-1, N}}, \ldots \boldsymbol{T}^{\star_{23} \star_{34} \ldots \star_{N-2, N-1} \star_{N-1, N}}, \boldsymbol{T}^{\star_{12} \star_{23} \star_{34} \ldots \star_{N-2, N-1} \star_{N-1, N}}\right], \\
& +(N-1) \text { cycle permutations of multistars in the }(N-1) \text { terms. }
\end{aligned}
$$

Proof. Insert (5.53) into (5.52) and use (5.46) together with $n_{T}$-ary associativity.

Definition 5.22. If all the polyadic projections (5.53) are equal to unity $\boldsymbol{P}_{\boldsymbol{T}}^{(k)}=\boldsymbol{I}$, then the corresponding polyadic isometry operator $\boldsymbol{T}$ is called a polyadic unitary operator.

It can be shown, that each polyadic unitary operator is querable ("polyadically invertible"), such that it has a querelement in $\mathcal{A}_{T}$.

5.3. Towards polyadic analog of $C^{*}$-algebras. Let us, first, generalize the operator binary norm (5.1) to the polyadic case. This can be done, provided that a binary ordering on the underlying polyadic field $\mathbb{K}_{m_{K}, n_{K}}$ can be introduced.

Definition 5.23. The polyadic operator norm $\|\bullet\|_{T}:\{\boldsymbol{T}\} \rightarrow K$ is defined by

$$
\|\boldsymbol{T}\|_{T}=\inf \left\{M \in K \mid\|\boldsymbol{T} \vee\|_{N} \leq \mu_{n_{K}}[\overbrace{M, \ldots, M}^{n_{K}-1},\|\mathrm{v}\|_{N}], \forall \mathrm{v} \in \mathrm{v}\right\},
$$

where $\|\bullet\|_{N}$ is the polyadic norm in the inner pairing space $\mathcal{H}_{m_{K}, n_{K}, m_{V}, k_{\rho}=1, N}$ and $\mu_{n_{K}}$ is the $n_{K}$-ary multiplication in $\mathbb{K}_{m_{K}, n_{K}}$.

Definition 5.24. The polyadic operator norm is called submultiplicative, if

$$
\begin{aligned}
\left\|\boldsymbol{\omega}_{n_{T}}\left[\boldsymbol{T}_{1}, \boldsymbol{T}_{2}, \ldots, \boldsymbol{T}_{n_{T}}\right]\right\|_{T} & \leq \mu_{n_{K}}\left[\left\|\boldsymbol{T}_{1}\right\|_{T},\left\|\boldsymbol{T}_{2}\right\|_{T}, \ldots,\left\|\boldsymbol{T}_{n_{K}}\right\|_{T}\right], \\
n_{T} & =n_{K} .
\end{aligned}
$$

Definition 5.25. The polyadic operator norm is called subadditive, if

$$
\begin{aligned}
\left\|\boldsymbol{\eta}_{m_{T}}\left[\boldsymbol{T}_{1}, \boldsymbol{T}_{2}, \ldots, \boldsymbol{T}_{n_{T}}\right]\right\|_{T} & \leq \nu_{m_{K}}\left[\left\|\boldsymbol{T}_{1}\right\|_{T},\left\|\boldsymbol{T}_{2}\right\|_{T}, \ldots,\left\|\boldsymbol{T}_{m_{K}}\right\|_{T}\right], \\
m_{T} & =m_{K} .
\end{aligned}
$$

By analogy with the binary case, we have

Definition 5.26. The polyadic operator algebra $\mathcal{A}_{T}$ equipped with the submultiplicative norm $\|\bullet\|_{T}$ a polyadic Banach algebra of operators $\mathcal{B}_{T}$.

The connection between the polyadic norms of operators and their polyadic adjoints is given by

Proposition 5.27. For polyadic operators in the inner pairing space $\mathcal{H}_{m_{K}, n_{K}, m_{V}, k_{\rho}=1, N}$ 
1) The following $N$ multi- $C^{*}$-relations

$$
\begin{aligned}
& \left\|\boldsymbol{\omega}_{n_{T}}\left[\boldsymbol{T}^{\star_{N-1, N}}, \boldsymbol{T}^{\star_{N-2, N-1} \star_{N-1, N}}, \ldots \boldsymbol{T}^{\star_{23} \star_{34} \ldots \star_{N-2, N-1} \star_{N-1, N}}, \boldsymbol{T}^{\star_{12} \star_{23} \star_{34} \ldots \star_{N-2, N-1} \star_{N-1, N}}, \boldsymbol{T}\right]\right\|_{N} \\
& =\mu_{n_{K}}[\overbrace{\|\boldsymbol{T}\|_{T},\|\boldsymbol{T}\|_{T}, \ldots,\|\boldsymbol{T}\|_{T}}^{n_{K}}] \text {, } \\
& +(N-1) \text { cycle permutations of }(N-1) \text { terms with multistars, } \\
& \text { take place, if } n_{T}=n_{K} \text {. }
\end{aligned}
$$

2) The polyadic norms of operator and its all adjoints coincide

$$
\left\|\boldsymbol{T}^{\star i, j}\right\|_{T}=\|\boldsymbol{T}\|_{T}, \forall i, j \in 1, \ldots, N .
$$

Proof. Both statements follow from (5.21) and the definition of the polyadic operator norm (5.55).

Therefore, we arrive to

Definition 5.28. The operator Banach algebra $\mathcal{B}_{T}$ satisfying the multi- $C^{*}$-relations is called a polyadic operator multi- $C^{*}$-algebra.

The first example of a multi- $C^{*}$-algebra (as in the binary case) can be constructed from one isometry operator (see Definition 5.15).

Definition 5.29. A polyadic algebra generated by one isometry operator $\boldsymbol{T}$ satisfying (5.46) on the inner pairing space $\mathcal{H}_{m_{K}, n_{K}, m_{V}, k_{\rho}=1, N}$ represents a polyadic Toeplitz algebra $\mathcal{T}_{m_{T}, n_{T}}$ and has the arity shape $m_{T}=m_{V}, n_{T}=N$.

Example 5.30. The ternary Toeplitz algebra $\mathcal{T}_{3,3}$ is represented by the operator $\boldsymbol{T}$ and relations

$$
\begin{aligned}
& {\left[\boldsymbol{T}^{\star_{1}}, \boldsymbol{T}^{\star_{3} \star_{1}}, \boldsymbol{T}\right]=\boldsymbol{I},} \\
& {\left[\boldsymbol{T}^{\star_{2}}, \boldsymbol{T}^{\star_{1} \star_{2}}, \boldsymbol{T}\right]=\boldsymbol{I},} \\
& {\left[\boldsymbol{T}^{\star_{3}}, \boldsymbol{T}^{\star_{2} \star_{3}}, \boldsymbol{T}\right]=\boldsymbol{I} .}
\end{aligned}
$$

Example 5.31. If the inner pairing is semicommutative (5.31), then $\left(\star_{3}\right)$ can be eliminated by

$$
\begin{aligned}
T^{\star_{3}} & =T^{\star_{1} \star_{2}}, \\
T^{\star_{3} \star_{3}} & =T,
\end{aligned}
$$

and the corresponding relations representing $\mathcal{T}_{3,3}$ become

$$
\begin{gathered}
{\left[\boldsymbol{T}^{\star_{1}}, \boldsymbol{T}^{\star_{1}}, \boldsymbol{T}\right]=\boldsymbol{I},} \\
{\left[\boldsymbol{T}_{\star_{2}}, \boldsymbol{T}_{\star_{1} \star_{2}}, \boldsymbol{T}\right]=\boldsymbol{I},} \\
{\left[\boldsymbol{T}^{\star_{1} \star_{2}}, \boldsymbol{T}^{\star_{2}}, \boldsymbol{T}\right]=\boldsymbol{I} .}
\end{gathered}
$$

Let us consider $M$ polyadic operators $\boldsymbol{T}_{1} \boldsymbol{T}_{2} \ldots \boldsymbol{T}_{M} \in \mathcal{B}_{T}$ and the related partial (in the usual sense) isometries (5.52) which are mutually orthogonal (5.50). In the binary case, the algebra generated by $M$ operators, such that the sum of the related orthogonal partial projections is unity, represents the Cuntz algebra $\mathcal{O}_{M}$ CUNTZ [1977].

Definition 5.32. A polyadic algebra generated by $M$ polyadic isometric operators $\boldsymbol{T}_{1} \boldsymbol{T}_{2} \ldots \boldsymbol{T}_{M} \in \mathcal{B}_{T}$ satisfying

$$
\boldsymbol{\eta}_{m_{T}}^{\left(\ell_{a}\right)}\left[\boldsymbol{P}_{\boldsymbol{T}_{1}}^{(k)}, \boldsymbol{P}_{\boldsymbol{T}_{2}}^{(k)} \ldots \boldsymbol{P}_{\boldsymbol{T}_{M}}^{(k)}\right]=\boldsymbol{I}, \quad k=1, \ldots, N
$$

where $\boldsymbol{P}_{\boldsymbol{T}_{i}}^{(k)}$ are given by (5.53) and $\boldsymbol{\eta}_{m_{T}}^{\left(\ell_{a}\right)}$ is a "long polyadic addition" (5.16), represents a polyadic Cuntz algebra $p \mathcal{O}_{M \mid m_{T}, n_{T}}$, which has the arity shape

$$
M=\ell_{a}\left(m_{T}-1\right)+1,
$$


where $\ell_{a}$ is number of " $m_{T}$-ary additions".

Below we use the same notations, as in Example 5.13, also the ternary addition will be denoted by $\left(+_{3}\right)$ as follows: $\boldsymbol{\eta}_{3}\left[\boldsymbol{T}_{1}, \boldsymbol{T}_{2}, \boldsymbol{T}_{3}\right] \equiv \boldsymbol{T}_{1}+{ }_{3} \boldsymbol{T}_{2}+{ }_{3} \boldsymbol{T}_{3}$.

Example 5.33. In the ternary case $m_{T}=n_{T}=3$ and one ternary addition $\ell_{a}=1$, we have $M=3$ mutually orthogonal isometries $\boldsymbol{T}_{1}, \boldsymbol{T}_{2}, \boldsymbol{T}_{3} \in \mathcal{B}_{T}$ and $N=3$ multistars $\left(\star_{i}\right)$. In case of the Post-like multistar cocycles (5.41) they satisfy

$$
\begin{array}{cc}
\text { Isometry conditions } & \text { Orthogonality conditions } \\
{\left[\boldsymbol{T}_{i}^{\star_{1}}, \boldsymbol{T}_{i}^{\star{ }^{\star} 1}, \boldsymbol{T}_{i}\right]=\boldsymbol{I},} & {\left[\boldsymbol{T}_{i}^{\star_{1}}, \boldsymbol{T}_{j}^{\star_{3}{ }_{1}}, \boldsymbol{T}_{k}\right]=\boldsymbol{Z},} \\
{\left[\boldsymbol{T}_{i}^{\star 2}, \boldsymbol{T}_{i}^{\star_{1}{ }_{2}}, \boldsymbol{T}_{i}\right]=\boldsymbol{I},} & {\left[\boldsymbol{T}_{i}^{\star_{2}}, \boldsymbol{T}_{j}^{\star_{1} \star_{2}}, \boldsymbol{T}_{k}\right]=\boldsymbol{Z},} \\
{\left[\boldsymbol{T}_{i}^{\star 3}, \boldsymbol{T}_{i}^{\star_{2}{ }_{3}}, \boldsymbol{T}_{i}\right]=\boldsymbol{I},} & {\left[\boldsymbol{T}_{i}^{\star_{3}}, \boldsymbol{T}_{j}^{\star_{2}{ }_{3}}, \boldsymbol{T}_{k}\right]=\boldsymbol{Z},} \\
i=1,2,3, & i, j, k=1,2,3, i \neq j \neq k, \quad i \neq k,
\end{array}
$$

and the (sum of projections) relations

$$
\begin{aligned}
& {\left[\boldsymbol{T}_{1}, \boldsymbol{T}_{1}^{\star_{1}}, \boldsymbol{T}_{1}^{\star_{3} \star_{1}}\right]+_{3}\left[\boldsymbol{T}_{2}, \boldsymbol{T}_{2}^{\star_{1}}, \boldsymbol{T}_{2}^{\star_{3} \star_{1}}\right]+_{3}\left[\boldsymbol{T}_{3}, \boldsymbol{T}_{3}^{\star_{1}}, \boldsymbol{T}_{3}^{\star_{3} \star_{1}}\right]=\boldsymbol{I},} \\
& {\left[\boldsymbol{T}_{1}, \boldsymbol{T}_{1}^{\star_{2}}, \boldsymbol{T}_{1}^{\star_{1} \star_{2}}\right]+_{3}\left[\boldsymbol{T}_{2}, \boldsymbol{T}_{2}^{\star_{2}}, \boldsymbol{T}_{2}^{\star_{1} \star_{2}}\right]+_{3}\left[\boldsymbol{T}_{3}, \boldsymbol{T}_{3}^{\star_{2}}, \boldsymbol{T}_{3}^{\star_{1} \star_{2}}\right]=\boldsymbol{I},} \\
& {\left[\boldsymbol{T}_{1}, \boldsymbol{T}_{1}^{\star_{3}}, \boldsymbol{T}_{1}^{\star_{2} \star_{3}}\right]+_{3}\left[\boldsymbol{T}_{2}, \boldsymbol{T}_{2}^{\star_{3}}, \boldsymbol{T}_{2}^{\star_{2} \star_{3}}\right]+_{3}\left[\boldsymbol{T}_{3}, \boldsymbol{T}_{3}^{\star_{3}}, \boldsymbol{T}_{3}^{\star_{2} \star_{3}}\right]=\boldsymbol{I},}
\end{aligned}
$$

which represent the ternary Cuntz algebra $p \mathcal{O}_{3 \mid 3,3}$.

Example 5.34. In the case where the inner pairing is semicommutative (5.31), we can eliminate the multistar $\left(\star_{3}\right)$ by (5.63) and represent the two-multistar ternary analog of the Cuntz algebra $p \mathcal{O}_{3 \mid 3,3}$ by

$$
\begin{aligned}
& {\left[\boldsymbol{T}_{i}^{\star_{1}}, \boldsymbol{T}_{i}^{\star_{2}}, \boldsymbol{T}_{i}\right]=\boldsymbol{I}, \quad\left[\boldsymbol{T}_{i}^{\star_{1}}, \boldsymbol{T}_{j}^{\star_{2}}, \boldsymbol{T}_{k}\right]=\boldsymbol{Z},} \\
& \begin{array}{ll}
{\left[\boldsymbol{T}_{i}^{\star 2}, \boldsymbol{T}_{i}^{\star \star_{2}}, \boldsymbol{T}_{i}\right]=\boldsymbol{I},} & {\left[\boldsymbol{T}_{i}^{\star_{1}}, \boldsymbol{T}_{j}^{\star_{1} \star_{2}}, \boldsymbol{T}_{k}\right]=\boldsymbol{Z},} \\
{\left[\boldsymbol{T}_{i}^{\star \star_{2}}, \boldsymbol{T}_{i}^{\star_{2}}, \boldsymbol{T}_{i}\right]=\boldsymbol{I},} & {\left[\boldsymbol{T}_{i}^{\star_{1}}, \boldsymbol{T}_{j}^{\star_{2}}, \boldsymbol{T}_{k}\right]=\boldsymbol{Z},}
\end{array} \\
& i=1,2,3, \quad i, j, k=1,2,3, \quad i \neq j \neq k, \\
& {\left[\boldsymbol{T}_{1}, \boldsymbol{T}_{1}^{\star 1}, \boldsymbol{T}_{1}^{\star 1}\right]+_{3}\left[\boldsymbol{T}_{2}, \boldsymbol{T}_{2}^{\star 1}, \boldsymbol{T}_{2}^{\star 1}\right]+_{3}\left[\boldsymbol{T}_{3}, \boldsymbol{T}_{3}^{\star 1}, \boldsymbol{T}_{3}^{\star 1}\right]=\boldsymbol{I},} \\
& {\left[\boldsymbol{T}_{1}, \boldsymbol{T}_{1}^{\star 2}, \boldsymbol{T}_{1}^{\star \star_{1}}\right]+{ }_{3}\left[\boldsymbol{T}_{2}, \boldsymbol{T}_{2}^{\star 2}, \boldsymbol{T}_{2}^{\star \star_{1}{ }_{2}}\right]+{ }_{3}\left[\boldsymbol{T}_{3}, \boldsymbol{T}_{3}^{\star 2}, \boldsymbol{T}_{3}^{\star 1 \star 2}\right]=\boldsymbol{I},} \\
& {\left[\boldsymbol{T}_{1}, \boldsymbol{T}_{1}^{\star 1 \star 2}, \boldsymbol{T}_{1}^{\star 2}\right]+_{3}\left[\boldsymbol{T}_{2}, \boldsymbol{T}_{2}^{\star 1 \star 2}, \boldsymbol{T}_{2}^{\star 2}\right]+_{3}\left[\boldsymbol{T}_{3}, \boldsymbol{T}_{3}^{\star 1 \star 2}, \boldsymbol{T}_{3}^{\star 2}\right]=\boldsymbol{I} \text {. }}
\end{aligned}
$$

\section{Congruence Classes as POLYADIC RINGS}

Here we will show that the inner structure of the residue classes (congruence classes) over integers is naturally described by polyadic rings CELAKOSKI [1977], CROMBEZ [1972], LEESON AND BUTSON [1980], and then study some special properties of them.

Denote a residue class (congruence class) of an integer $a$, modulo $b$ by

$$
[[a]]_{b}=\left\{\{a+b k\} \mid k \in \mathbb{Z}, a \in \mathbb{Z}_{+}, b \in \mathbb{N}, 0 \leq a \leq b-1\right\} .
$$

A representative element of the class $[[a]]_{b}$ will be denoted by $x_{k}=x_{k}^{(a, b)}=a+b k$. Here we do not consider the addition and multiplication of the residue classes (congruence classes). Instead, we consider the fixed congruence class $[[a]]_{b}$, and note that, for arbitrary $a$ and $b$, it is not closed under binary operations. However, it can be closed with respect to polyadic operations.

\footnotetext{
${ }^{3}$ We use for the residue class the notation $[[a]]_{b}$, because the standard notations by one square bracket $[a]_{b}$ or $\bar{a}_{b}$ are busy by the $n$-ary operations and querelements, respectively.
} 
6.1. Polyadic ring on integers. Let us introduce the $m$-ary addition and $n$-ary multiplication of representatives of the fixed congruence class $[[a]]_{b}$ by

$$
\begin{aligned}
\nu_{m}\left[x_{k_{1}}, x_{k_{2}}, \ldots, x_{k_{m}}\right] & =x_{k_{1}}+x_{k_{2}}+\ldots+x_{k_{m}}, \\
\mu_{n}\left[x_{k_{1}}, x_{k_{2}}, \ldots, x_{k_{n}}\right] & =x_{k_{1}} x_{k_{2}} \ldots x_{k_{n}}, \quad x_{k_{i}} \in[[a]]_{b}, k_{i} \in \mathbb{Z},
\end{aligned}
$$

where on the r.h.s. the operations are the ordinary binary addition and binary multiplication in $\mathbb{Z}$.

Remark 6.1. The polyadic operations (6.2)-(6.3) are not derived (see, e.g., GŁAZEK AND MICHALSKI [1984], MICHALSKI [1988]), because on the set $\left\{x_{k_{i}}\right\}$ one cannot define the binary semigroup structure with respect to ordinary addition and multiplication. Derived polyadic rings which consist of the repeated binary sums and binary products were considered in LEESON AND BUTSON [1980].

Lemma 6.2. In case

$$
(m-1) \frac{a}{b}=I^{(m)}(a, b)=I=\text { integer }
$$

the algebraic structure $\left\langle[[a]]_{b} \mid \nu_{m}\right\rangle$ is a commutative m-ary group.

Proof. The closure of the operation (6.2) can be written as $x_{k_{1}}+x_{k_{2}}+\ldots+x_{k_{m}}=x_{k_{0}}$, or $m a+$ $b\left(k_{1}+k_{2}+\ldots+k_{m}\right)=a+b k_{0}$, and then $k_{0}=(m-1) a / b+\left(k_{1}+k_{2}+\ldots+k_{m}\right)$, from (6.4). The (total) associativity and commutativity of $\nu_{m}$ follows from those of the addition in the binary $\mathbb{Z}$. Each element $x_{k}$ has its unique querelement $\tilde{x}=x_{\tilde{k}}$ determined by the equation $(m-1) x_{k}+x_{\tilde{k}}=x_{k}$, which (uniquely, for any $k \in \mathbb{Z}$ ) gives

$$
\tilde{k}=b k(2-m)-(m-1) \frac{a}{b} .
$$

Thus, each element is "querable" (polyadic invertible), and so $\left\langle[[a]]_{b} \mid \nu_{m}\right\rangle$ is a $m$-ary group.

Example 6.3. For $a=2, b=7$ we have 8-ary group, and the querelement of $x_{k}$ is $\tilde{x}=x_{(-2-12 k)}$.

Proposition 6.4. The m-ary commutative group $\left\langle[[a]]_{b} \mid \nu_{m}\right\rangle$ :

1) has an infinite number of neutral sequences for each element;

2) if a $\neq 0$, it has no "unit" (which is actually zero, because $\nu_{m}$ plays the role of "addition");

3) in case of the zero congruence class $[[0]]_{b}$ the zero is $x_{k}=0$.

Proof.

1) The (additive) neutral sequence $\tilde{\boldsymbol{n}}_{m-1}$ of the length $(m-1)$ is defined by $\nu_{m}\left[\tilde{\boldsymbol{n}}_{m-1}, x_{k}\right]=x_{k}$. Using (6.2), we take $\tilde{\boldsymbol{n}}_{m-1}=x_{k_{1}}+x_{k_{2}}+\ldots+x_{k_{m-1}}=0$ and obtain the equation

$$
(m-1) a+b\left(k_{1}+k_{2}+\ldots+k_{m-1}\right)=0 .
$$

Because of (6.4), we obtain

$$
k_{1}+k_{2}+\ldots+k_{m-1}=-I^{(m)}(a, b),
$$

and so there is an infinite number of sums satisfying this condition.

2) The polyadic "unit"/zero $z=x_{k_{0}}=a+b k_{0}$ satisfies $\nu_{m}[\overbrace{z, z, \ldots, z}^{m-1} x_{k}]=x_{k}$ for all $x_{k} \in[[a]]_{b}$ (the neutral sequence $\tilde{\boldsymbol{n}}_{m-1}$ consists of one element $z$ only), which gives $(m-1)\left(a+b k_{0}\right)=0$ having no solutions with $a \neq 0$, since $a<b$.

3) In the case $a=0$, the only solution is $z=x_{k=0}=0$.

Example 6.5. In case $a=1, b=2$ we have $m=3$ and $I^{(3)}(1,2)=1$, and so from (6.6) we get $k_{1}+k_{2}=-1$, thus the infinite number of neutral sequences consists of 2 elements $\tilde{\boldsymbol{n}}_{2}=x_{k}+x_{-1-k}$, with arbitrary $k \in \mathbb{Z}$. 
Lemma 6.6. If

$$
\frac{a^{n}-a}{b}=J^{(n)}(a, b)=J=\text { integer, }
$$

then $\left\langle[[a]]_{b} \mid \mu_{n}\right\rangle$ is a commutative n-ary semigroup.

Proof. It follows from (6.3), that the closeness of the operation $\mu_{n}$ is $x_{k_{1}} x_{k_{2}} \ldots x_{k_{n}}=x_{k_{0}}$, which can be written as $a^{n}+b$ (integer) $=a+b k_{0}$ leading to (6.8). The (total) associativity and commutativity of $\mu_{n}$ follows from those of the multiplication in $\mathbb{Z}$.

Definition 6.7. A unique pair of integers $(I, J)$ is called a (polyadic) shape invariants of the congruence class $[[a]]_{b}$.

Theorem 6.8. The algebraic structure of the fixed congruence class $[[a]]_{b}$ is a polyadic $(m, n)$-ring

$$
\mathcal{R}_{m, n}^{[a, b]}=\left\langle[[a]]_{b} \mid \nu_{m}, \mu_{n}\right\rangle,
$$

where the arities $m$ and $n$ are minimal positive integers (more or equal 2 ), for which the congruences

$$
\begin{aligned}
m a & \equiv a(\bmod b), \\
a^{n} & \equiv a(\bmod b)
\end{aligned}
$$

take place simultaneously, fixating its polyadic shape invariants $(I, J)$.

Proof. By Lemma 6.2, 6.6 the set $[[a]]_{b}$ is a $m$-ary group with respect to " $m$-ary addition" $\nu_{m}$ and a $n$ ary semigroup with respect to " $n$-ary multiplication" $\mu_{n}$, while the polyadic distributivity (1.1)-(1.3) follows from (6.2) and (6.3) and the binary distributivity in $\mathbb{Z}$.

Remark 6.9. For a fixed $b \geq 2$ there are $b$ congruence classes $[[a]]_{b}, 0 \leq a \leq b-1$, and therefore exactly $b$ corresponding polyadic $(m, n)$-rings $\mathcal{R}_{m, n}^{[a, b]}$, each of them is infinite-dimensional.

Corollary 6.10. In case $\operatorname{gcd}(a, b)=1$ and $b$ is prime, there exists the solution $n=b$.

Proof. Follows from (6.11) and Fermat's little theorem.

Remark 6.11. We exclude from consideration the zero congruence class $[[0]]_{b}$, because the arities of operations $\nu_{m}$ and $\mu_{n}$ cannot be fixed up by (6.10) -6.11) becoming identities for any $m$ and $n$. Since the arities are uncertain, their minimal values can be chosen $m=n=2$, and therefore, it follows from (6.2) and (6.3), $\mathcal{R}_{2,2}^{[0, b]}=\mathbb{Z}$. Thus, in what follows we always imply that $a \neq 0$ (without using a special notation, e.g. $\mathcal{R}^{*}$, etc.).

In TABLE 3 we present the allowed (by (6.10)-(6.11) arities of the polyadic ring $\mathcal{R}_{m, n}^{[a, b]}$ and the corresponding polyadic shape invariants $(I, J)$ for $b \leq 10$.

Let us investigate the properties of $\mathcal{R}_{m, n}^{[a, b]}$ in more detail. First, we consider equal arity polyadic rings and find the relation between the corresponding congruence classes.

Proposition 6.12. The residue (congruence) classes $[[a]]_{b}$ and $\left[\left[a^{\prime}\right]\right]_{b^{\prime}}$ which are described by the polyadic rings of the same arities $\mathcal{R}_{m, n}^{[a, b]}$ and $\mathcal{R}_{m, n}^{\left[a^{\prime}, b^{\prime}\right]}$ are related by

$$
\begin{aligned}
\frac{b^{\prime} I^{\prime}}{a^{\prime}} & =\frac{b I}{a}, \\
a^{\prime}+b^{\prime} J^{\prime} & =(a+b J)^{\log _{a} a^{\prime}} .
\end{aligned}
$$

Proof. Follows from (6.4) and (6.8).

For instance, in TABLE 3 the congruence classes $[[2]]_{5},[[3]]_{5},[[2]]_{10}$, and $[[8]]_{10}$ are $(6,5)$-rings. If, in addition, $a=a^{\prime}$, then the polyadic shapes satisfy

$$
\frac{I}{J}=\frac{I^{\prime}}{J^{\prime}}
$$


6.2. Limiting cases. The limiting cases $a \equiv \pm 1(\bmod b)$ are described by

Corollary 6.13. The polyadic ring of the fixed congruence class $[[a]]_{b}$ is: 1) multiplicative binary, if $a=1 ; 2)$ multiplicative ternary, if $a=b-1 ; \mathbf{3})$ additive $(b+1)$-ary in both cases. That is, the limiting cases contain the rings $\mathcal{R}_{b+1,2}^{[1, b]}$ and $\mathcal{R}_{b+1,3}^{[b-1, b]}$, respectively. They correspond to the first row and the main diagonal of TABLE 3. Their intersection consists of the $(3,2)$-ring $\mathcal{R}_{3,2}^{[1,2]}$.

Definition 6.14. The congruence classes $[[1]]_{b}$ and $[[b-1]]_{b}$ are called the limiting classes, and the corresponding polyadic rings are named the limiting polyadic rings of a fixed congruence class.

Proposition 6.15. In the limiting cases $a=1$ and $a=b-1$ the n-ary semigroup $\left\langle[[a]]_{b} \mid \mu_{n}\right\rangle$ :

1) has the neutral sequences of the form $\overline{\boldsymbol{n}}_{n-1}=x_{k_{1}} x_{k_{2}} \ldots x_{k_{n-1}}=1$, where $x_{k_{i}}= \pm 1$;

2) has a) the unit $e=x_{k=1}=1$, for the limiting class $\left.[[1]]_{b}, b\right)$ the unit $e^{-}=x_{k=-1}=-1$, if $n$ is odd, for $\left.[[b-1]]_{b}, c\right)$ the class $[[1]]_{2}$ contains both polyadic units $e$ and $e^{-}$;

3) has the set of "querable" (polyadic invertible) elements which consist of $\bar{x}=x_{\bar{k}}= \pm 1$;

4) has in the "intersecting" case $a=1, b=2$ and $n=2$ the binary subgroup $\mathbb{Z}_{2}=\{1,-1\}$, while other elements have no inverses.

Proof.

1) The (multiplicative) neutral sequence $\overline{\boldsymbol{n}}_{n-1}$ of length $(n-1)$ is defined by $\mu_{n}\left[\overline{\boldsymbol{n}}_{n-1}, x_{k}\right]=x_{k}$. It follows from (6.3) and cancellativity in $\mathbb{Z}$, that $\overline{\boldsymbol{n}}_{n-1}=x_{k_{1}} x_{k_{2}} \ldots x_{k_{n-1}}=1$ which is

$$
\left(a+b k_{1}\right)\left(a+b k_{2}\right) \ldots\left(a+b k_{n-1}\right)=1 .
$$

The solution of this equation in integers is the following: a) all multipliers are $a+b k_{i}=1$, $i=1, \ldots, n-1 ;$ b) an even number of multipliers can be $a+b k_{i}=-1$, while the others are 1.

2) If the polyadic unit $e=x_{k_{1}}=a+b k_{1}$ exists, it should satisfy $\mu_{m}[\overbrace{e, e, \ldots, e}^{n-1}, x_{k}]=x_{k}$ $\forall x_{k} \in\left\langle[[a]]_{b} \mid \mu_{n}\right\rangle$, such that the neutral sequence $\overline{\boldsymbol{n}}_{n-1}$ consists of one element $e$ only, and this leads to $\left(a+b k_{1}\right)^{n-1}=1$. For any $n$ this equation has the solution $a+b k_{1}=1$, which uniquely gives $a=1$ and $k_{1}=0$, thus $e=x_{k_{1}=0}=1$. If $n$ is odd, then there exists a "negative unit" $e^{-}=x_{k_{1}=-1}=-1$, such that $a+b k_{1}=-1$, which can be uniquely solved by $k_{1}=-1$ and $a=b-1$. The neutral sequence becomes $\overline{\boldsymbol{n}}_{n-1}=\overbrace{e^{-}, e^{-}, \ldots, e^{-}}^{n-1}=1$, as a product of an even number of $e^{-}=-1$. The intersection of limiting classes consists of one class $[[1]]_{2}$, and therefore it contains both polyadic units $e$ and $e^{-}$.

3) An element $x_{k}$ in $\left\langle[[a]]_{b} \mid \mu_{n}\right\rangle$ is "querable", if there exists its querelement $\bar{x}=x_{\bar{k}}$ such that $\mu_{n}[\overbrace{x_{k}, x_{k}, \ldots, x_{k}}^{n-1}, \bar{x}]=x_{k}$. Using (6.3) and the cancellativity in $\mathbb{Z}$, we obtain the equation $(a+b k)^{n-2}(a+b \bar{k})=1$, which in integers has 2 solutions: a) $(a+b k)^{n-2}=1$ and $(a+b \bar{k})=1$, the last relation fixes up the class $[[1]]_{b}$, and the arity of multiplication $n=2$, and therefore the first relation is valid for all elements in the class, each of them has the same querelement $\bar{x}=1$. This means that all elements in $[[1]]_{b}$ are "querable", but only one element $x=1$ has an inverse, which is also $1 ; \mathrm{b})(a+b k)^{n-2}=-1$ and $(a+b \bar{k})=-1$. The second relation fixes the class $[[b-1]]_{b}$, and from the first relation we conclude that the arity $n$ should be odd. In this case only one element -1 is "querable", which has $\bar{x}=-1$, as a querelement.

4) The "intersecting" class $[[1]]_{2}$ contains 2 "querable" elements \pm 1 which coincide with their inverses, which means that $\{+1,-1\}$ is a binary subgroup (that is $\mathbb{Z}_{2}$ ) of the binary semigroup $\left\langle[[1]]_{2} \mid \mu_{2}\right\rangle$. 
Corollary 6.16. In the non-limiting cases $a \neq 1, b-1$, the $n$-ary semigroup $\left\langle[[a]]_{b} \mid \mu_{n}\right\rangle$ contains no "querable" (polyadic invertible) elements at all.

Proof. It follows from $(a+b k) \neq \pm 1$ for any $k \in \mathbb{Z}$ or $a \neq \pm 1(\bmod b)$.

TABLE 3. The polyadic ring $\mathcal{R}_{m, n}^{\mathbb{Z}(a, b)}$ of the fixed residue class $[[a]]_{b}$ : arity shape.

\begin{tabular}{|c|c|c|c|c|c|c|c|c|c|}
\hline$a \backslash b$ & 2 & 3 & 4 & 5 & 6 & 7 & 8 & 9 & 10 \\
\hline 1 & $\begin{aligned} m & =\mathbf{3} \\
n & =\mathbf{2} \\
I & =1 \\
J & =0\end{aligned}$ & $\begin{aligned} m & =\mathbf{4} \\
n & =\mathbf{2} \\
I & =1 \\
J & =0\end{aligned}$ & $\begin{aligned} m & =\mathbf{5} \\
n & =\mathbf{2} \\
I & =1 \\
J & =0\end{aligned}$ & $\begin{aligned} m & =\mathbf{6} \\
n & =\mathbf{2} \\
I & =1 \\
J & =0\end{aligned}$ & $\begin{array}{l}m=\mathbf{7} \\
n=\mathbf{2} \\
I=1 \\
J=0\end{array}$ & $\begin{array}{l}m=\mathbf{8} \\
n=\mathbf{2} \\
I=1 \\
J=0\end{array}$ & $\begin{aligned} m & =\mathbf{9} \\
n & =\mathbf{2} \\
I & =1 \\
J & =0\end{aligned}$ & $\begin{array}{c}m=\mathbf{1 0} \\
n=\mathbf{2} \\
I=1 \\
J=0\end{array}$ & $\begin{array}{c}m=\mathbf{1 1} \\
n=\mathbf{2} \\
I=1 \\
J=0\end{array}$ \\
\hline 2 & & $\begin{array}{l}m=\mathbf{4} \\
n=\mathbf{3} \\
I=2 \\
J=2\end{array}$ & & $\begin{aligned} m & =\mathbf{6} \\
n & =\mathbf{5} \\
I & =2 \\
J & =6\end{aligned}$ & $\begin{aligned} m & =\mathbf{4} \\
n & =\mathbf{3} \\
I & =1 \\
J & =1\end{aligned}$ & $\begin{array}{c}m=\mathbf{8} \\
n=4 \\
I=2 \\
J=2\end{array}$ & & $\begin{array}{c}m=\mathbf{1 0} \\
n=\mathbf{7} \\
I=2 \\
J=14\end{array}$ & $\begin{array}{c}m=\mathbf{6} \\
n=\mathbf{5} \\
I=1 \\
J=3\end{array}$ \\
\hline 3 & & & $\begin{aligned} m & =\mathbf{5} \\
n & =\mathbf{3} \\
I & =3 \\
J & =6\end{aligned}$ & $\begin{array}{c}m=\mathbf{6} \\
n=\mathbf{5} \\
I=3 \\
J=48\end{array}$ & $\begin{aligned} m & =\mathbf{3} \\
n & =\mathbf{2} \\
I & =1 \\
J & =1\end{aligned}$ & $\begin{array}{c}m=\mathbf{8} \\
n=\mathbf{7} \\
I=3 \\
J=312\end{array}$ & $\begin{aligned} m & =\mathbf{9} \\
n & =\mathbf{3} \\
I & =3 \\
J & =3\end{aligned}$ & & $\begin{array}{c}m=\mathbf{1 1} \\
n=\mathbf{5} \\
I=3 \\
J=24\end{array}$ \\
\hline 4 & & & & $\begin{array}{c}m=\mathbf{6} \\
n=\mathbf{3} \\
I=4 \\
J=12\end{array}$ & $\begin{aligned} m & =\mathbf{4} \\
n & =\mathbf{2} \\
I & =2 \\
J & =2\end{aligned}$ & $\begin{array}{c}m=8 \\
n=4 \\
I=4 \\
J=36\end{array}$ & & $\begin{array}{c}m=\mathbf{1 0} \\
n=\mathbf{4} \\
I=4 \\
J=28\end{array}$ & $\begin{aligned} m & =\mathbf{6} \\
n & =\mathbf{3} \\
I & =2 \\
J & =6\end{aligned}$ \\
\hline 5 & & & & & $\begin{aligned} m & =\mathbf{7} \\
n & =\mathbf{3} \\
I & =5 \\
J & =20\end{aligned}$ & $\begin{array}{c}m=\mathbf{8} \\
n=\mathbf{7} \\
I=5 \\
J=11160\end{array}$ & $\begin{array}{c}m=\mathbf{9} \\
n=\mathbf{3} \\
I=5 \\
J=15\end{array}$ & $\begin{array}{c}m=10 \\
n=7 \\
I=5 \\
J=8680\end{array}$ & $\begin{aligned} m & =\mathbf{3} \\
n & =\mathbf{2} \\
I & =1 \\
J & =2\end{aligned}$ \\
\hline 6 & & & & & & $\begin{array}{c}m=\mathbf{8} \\
n=\mathbf{3} \\
I=6 \\
J=30\end{array}$ & & & $\begin{array}{c}m=\mathbf{6} \\
n=\mathbf{2} \\
I=3 \\
J=3\end{array}$ \\
\hline 7 & & & & & & & $\begin{aligned} m & =\mathbf{9} \\
n & =\mathbf{3} \\
I & =7 \\
J & =42\end{aligned}$ & $\begin{array}{c}m=\mathbf{1 0} \\
n=4 \\
I=7 \\
J=266\end{array}$ & $\begin{array}{c}m=\mathbf{1 1} \\
n=\mathbf{5} \\
I=7 \\
J=1680\end{array}$ \\
\hline 8 & & & & & & & & $\begin{aligned} m & =\mathbf{1 0} \\
n & =\mathbf{3} \\
I & =8 \\
J & =56\end{aligned}$ & $\begin{array}{c}m=\mathbf{6} \\
n=\mathbf{5} \\
I=4 \\
J=3276\end{array}$ \\
\hline 9 & & & & & & & & & $\begin{aligned} m & =\mathbf{1 1} \\
n & =\mathbf{3} \\
I & =9 \\
J & =72\end{aligned}$ \\
\hline
\end{tabular}

Based on the above statements, consider in the properties of the polyadic rings $\mathcal{R}_{m, n}^{[a, b]}(a \neq 0)$ describing non-zero congruence classes (see Remark 6.11).

Definition 6.17. The infinite set of representatives of the congruence (residue) class $[[a]]_{b}$ having fixed arities and form the $(m, n)$-ring $\mathcal{R}_{m, n}^{[a, b]}$ is called the set of (polyadic) $(m, n)$-integers (numbers) and denoted $\mathbb{Z}_{(m, n)}$.

Just obviously, for ordinary integers $\mathbb{Z}=\mathbb{Z}_{(2,2)}$, and they form the binary ring $\mathcal{R}_{2,2}^{[0,1]}$.

Proposition 6.18. The polyadic ring $\mathcal{R}_{m, n}^{[a, b]}$ is a $(m, n)$-integral domain.

Proof. It follows from the definitions $(6.2)-(6.3)$, the condition $a \neq 0$, and commutativity and cancellativity in $\mathbb{Z}$. 
Lemma 6.19. There are no such congruence classes which can be described by polyadic ( $m, n)$-field.

Proof. Follows from Proposition 6.15 and Corollary 6.16.

This statement for the limiting case $[[1]]_{2}$ appeared in DUPLIJ AND WERNER [2015], while studying the ideal structure of the corresponding $(3,2)$-ring.

Proposition 6.20. In the limiting case $a=1$ the polyadic ring $\mathcal{R}_{b+1,2}^{[1, b]}$ can be embedded into a $(b+1,2)$-ary field.

Proof. Because the polyadic ring $\mathcal{R}_{b+1,2}^{[1, b]}$ of the congruence class $[[1]]_{b}$ is an $(b+1,2)$-integral domain by Proposition 6.18, we can construct in a standard way the correspondent $(b+1,2)$-quotient ring which is a $(b+1,2)$-ary field up to isomorphism, as was shown in CROMBEZ AND TIMM [1972]. By analogy, it can be called the field of polyadic rational numbers which have the form

$$
x=\frac{1+b k_{1}}{1+b k_{2}}, \quad k_{i} \in \mathbb{Z} .
$$

Indeed, they form a $(b+1,2)$-field, because each element has its inverse under multiplication (which is obvious) and additively "querable", such that the equation for the querelement $\bar{x}$ becomes $\nu_{b+1}[\overbrace{x, x, \ldots, x}^{b}, \bar{x}]=x$ which can be solved for any $x$, giving uniquely $\bar{x}=-(b-1) \frac{1+b k_{1}}{1+b k_{2}}$.

The introduced polyadic inner structure of the residue (congruence) classes allows us to extend various number theory problems by considering the polyadic $(m, n)$-integers $\mathbb{Z}_{(m, n)}$ instead of $\mathbb{Z}$.

\section{EQUAL SUMS OF LIKE POWERS DIOPHANTINE EQUATION OVER POLYADIC INTEGERS}

First, recall the standard binary version of the equal sums of like powers Diophantine equation LANDER ET AL. [1967], EKL [1998]. Take the fixed non-negative integers $p, q, l \in \mathbb{N}^{0}, p \leq q$, and the positive integer unknowns $u_{i}, v_{j} \in \mathbb{Z}_{+}, i=1, \ldots p+1, j=1,1, \ldots q+1$, then the Diophantine equation is

$$
\sum_{i=1}^{p+1} u_{i}^{l+1}=\sum_{j=1}^{q+1} v_{j}^{l+1}
$$

The trivial case, when $u_{i}=0, v_{j}=0$, for all $i, j$ is not considered. We mark the solutions of (7.1) by the triple $(l \mid p, q)_{r}$ showing quantity of operations 4 , where $r$ (if it is used) is the order of the solution (ranked by the value of the sum) and the unknowns $u_{i}, v_{j}$ are placed in ascending order $u_{i} \leq u_{i+1}, v_{j} \leq v_{j+1}$.

Let us recall the Tarry-Escott problem (or multigrades problem) DORWART AND BROWN [1937]: to find the solutions to (7.1) for an equal number of summands on both sides of $p=q$ and $s$ equations simultaneously, such that $l=0, \ldots, s$. Known solutions exist for powers until $s=10$, which are bounded such that $s \leq p$ (in our notations), see, also, NGUYEN [2016]. The solutions with highest powers $s=p$ are the most interesting and called the ideal solutions BORWEIN [2002].

Theorem 7.1 (Frolov Frolov [1889]). If the set of $s$ Diophantine equations (7.1) with $p=q$ for $l=0, \ldots, s$ has a solution $\left\{u_{i}, v_{i}, i=1, \ldots p+1\right\}$, then it has the solution $\left\{a+b u_{i}, a+b v_{i}, i=1, \ldots p+1\right\}$, where $a, b \in \mathbb{Z}$ are arbitrary and fixed.

\footnotetext{
${ }^{4}$ In the binary case, the solutions of (7.1) are usually denoted by $(l+1 \mid p+1, q+1)_{r}$, which shows the number of summands on both sides and powers of elements LANDER ET AL. [1967]. But in the polyadic case (see below), the number of summands and powers do not coincide with $l+1, p+1, q+1$, at all.
} 
In the simplest case $(1 \mid 0,1)$, one term in 1.h.s., one addition on the r.h.s. and one multiplication, the (coprime) positive numbers satisfying (7.1) are called a (primitive) Pythagorean triple. For the Fermat's triple $(l \mid 0,1)$ with one addition on the r.h.s. and more than one multiplication $l \geq 2$, there are no solutions of (7.1), which is known as Fermat's last theorem proved in WILES [1995]. There are many solutions known with more than one addition on both sides, where the highest number of multiplications till now is 31 (S. Chase, 2012).

Before generalizing (7.1) for polyadic case we note the following.

Remark 7.2. The notations in (7.1) are chosen in such a way that $p$ and $q$ are numbers of binary additions on both sides, while $l$ is the number of binary multiplications in each term, which is natural for using polyadic powers DUPLIJ [2012].

7.1. Polyadic analog of the Lander-Parkin-Selfridge conjecture. In LANDER ET AL. [1967], a generalization of Fermat's last theorem was conjectured, that the solutions of (7.1) exist for small powers only, which can be formulated in terms of the numbers of operations as

Conjecture 7.3 (Lander-Parkin-Selfridge LANDER ET AL. [1967]). There exist solutions of (7.1) in positive integers, if the number of multiplications is less than or equal than the total number of additions plus one

$$
3 \leq l \leq l_{L S P}=p+q+1
$$

where $p+q \geq 2$.

Remark 7.4. If the equation (7.1) is considered over the binary ring of integers $\mathbb{Z}$, such that $u_{i}, v_{j} \in \mathbb{Z}$, it leads to a straightforward reformulation: for even powers it is obvious, but for odd powers all negative terms can be rearranged and placed on the other side.

Let us consider the Diophantine equation (7.1) over polyadic integers $\mathbb{Z}_{(m, n)}$ (i.e. over the polyadic $(m, n)$-ary ring $\mathcal{R}_{m, n}^{\mathbb{Z}}$ ) such that $u_{i}, v_{j} \in \mathcal{R}_{m, n}^{\mathbb{Z}}$. We use the "long products" $\mu_{n}^{(l)}$ and $\nu_{m}^{(l)}$ containing $l$ operations, and also the "polyadic power" for an element $x \in \mathcal{R}_{m, n}^{\mathbb{Z}}$ with respect to $n$-ary multiplication DUPLIJ [2012]

$$
x^{\langle l\rangle_{n}}=\mu_{n}^{(l)}[\overbrace{x, x, \ldots, x}^{l(n-1)+1}] .
$$

In the binary case, $n=2$, the polyadic power coincides with $(l+1)$ power of an element $x^{\langle l\rangle_{2}}=x^{l+1}$, which explains REMARK 7.2. In this notation the polyadic analog of the equal sums of like powers Diophantine equation has the form

$$
\nu_{m}^{(p)}\left[u_{1}^{\langle l\rangle_{n}}, u_{2}^{\langle l\rangle_{n}}, \ldots, u_{p(m-1)+1}^{\langle l\rangle_{n}}\right]=\nu_{m}^{(q)}\left[v_{1}^{\langle l\rangle_{n}}, v_{2}^{\langle l\rangle_{n}}, \ldots, v_{q(m-1)+1}^{\langle l\rangle_{n}}\right],
$$

where $p$ and $q$ are number of $m$-ary additions in 1.h.s. and r.h.s. correspondingly. The solutions of (7.4) will be denoted by $\left\{u_{1}, u_{2}, \ldots, u_{p(m-1)+1} ; v_{1}, v_{2}, \ldots, v_{q(m-1)+1}\right\}$. In the binary case $m=2, n=2$, (7.4) reduces to (7.1). Analogously, we mark the solutions of (7.4) by the polyadic triple $(l \mid p, q)_{r}^{(m, n)}$. Now the polyadic Pythagorean triple $(1 \mid 0,1)^{(m, n)}$, having one term on the 1.h.s., one $m$-ary addition on the r.h.s. and one $n$-ary multiplication (elements are in the first polyadic power $\langle 1\rangle_{n}$ ), becomes

$$
u_{1}^{\langle 1\rangle_{n}}=\nu_{m}\left[v_{1}^{\langle 1\rangle_{n}}, v_{2}^{\langle 1\rangle_{n}}, \ldots, v_{m}^{\langle 1\rangle_{n}}\right]
$$

Definition 7.5. The equation (7.5) solved by minimal $u_{1}, v_{i} \in \mathbb{Z}, i=1, \ldots, m$ can be named the polyadic Pythagorean theorem. 
The polyadic Fermat's triple $(l \mid 0,1)^{(m, n)}$ has one term in 1.h.s., one $m$-ary addition in r.h.s. and $l$ (n-ary) multiplications

$$
u_{1}^{\langle l\rangle_{n}}=\nu_{m}\left[v_{1}^{\langle l\rangle_{n}}, v_{2}^{\langle l\rangle_{n}}, \ldots, v_{m}^{\langle l\rangle_{n}}\right] .
$$

One may be interested in whether the polyadic analog of Fermat's last theorem is valid, and if not, in which cases the analogy with the binary case can be sustained.

Conjecture 7.6 (Polyadic analog of Fermat's Last Theorem). The polyadic Fermat's triple (7.6) has no solutions over the polyadic $(m, n)$-ary $\operatorname{ring} \mathcal{R}_{m, n}^{\mathbb{Z}}$, if $l \geq 2$, i.e. there are more than one $n$-ary multiplications.

Its straightforward generalization leads to the polyadic version of the Lander-Parkin-Selfridge conjecture, as

Conjecture 7.7 (Polyadic Lander-Parkin-Selfridge conjecture). There exist solutions of the polyadic analog of the equal sums of like powers Diophantine equation (7.4) in integers, if the number of $n$-ary multiplications is less than or equal than the total number of $m$-ary additions plus one

$$
3 \leq l \leq l_{p L P S}=p+q+1 .
$$

Below we will see a counterexample to both of the above conjectures.

Example 7.8. Let us consider the (3,2)-ring $\mathcal{R}_{3,2}^{\mathbb{Z}}=\left\langle\mathbb{Z} \mid \nu_{3}, \mu_{2}\right\rangle$, where

$$
\begin{aligned}
\nu_{3}[x, y, z] & =x+y+z+2, \\
\mu_{2}[x, y] & =x y+x+y .
\end{aligned}
$$

Note that this exotic polyadic ring is commutative and cancellative, having unit 0 , no multiplicative inverses, and for any $x \in \mathcal{R}_{3,2}^{\mathbb{Z}}$ its additive querelement $\tilde{x}=-x-2$, therefore $\left\langle\mathbb{Z} \mid \nu_{3}\right\rangle$ is a ternary group (as it should be). The polyadic power of any element is

$$
x^{\langle l\rangle_{2}}=(x+1)^{l+1}-1 .
$$

1) For $\mathcal{R}_{3,2}^{\mathbb{Z}}$ the polyadic Pythagorean triple $(1 \mid 0,1)^{(3,2)}$ in (7.5) now is

$$
u^{\langle 1\rangle_{2}}=\nu_{3}\left[x^{\langle 1\rangle_{2}}, y^{\langle 1\rangle_{2}}, z^{\langle 1\rangle_{2}}\right],
$$

which, using (7.3), (7.9) and (7.10), becomes the (shifted) Pythagorean quadruple SPIRA [1962]

$$
(u+1)^{2}=(x+1)^{2}+(y+1)^{2}+(z+1)^{2},
$$

and it has infinite number of solutions, among which two minimal ones $\{u=2 ; x=0, y=z=1\}$ and $\{u=14 ; x=1, y=9, z=10\}$ give $3^{2}=1^{2}+2^{2}+2^{2}$ and $15^{2}=2^{2}+10^{2}+11^{2}$, correspondingly.

2) For this $(3,2)$-ring $\mathcal{R}_{3,2}^{\mathbb{Z}}$ the polyadic Fermat's triple $(l \mid 0,1)^{(3,2)}$ becomes

$$
(u+1)^{l+1}=(x+1)^{l+1}+(y+1)^{l+1}+(z+1)^{l+1} .
$$

If the polyadic analog of Fermat's last theorem 7.6 holds, then there are no solutions to (7.13) for more than one $n$-ary multiplication $l \geq 2$. But this is the particular case, $p=0, q=2$, of the binary Lander-Parkin-Selfridge Conjecture 7.3 which now takes the form: the solutions to (7.13) exist, if $l \leq 3$. Thus, as a counterexample to the polyadic analog of Fermat's last theorem, we have two possible solutions with numbers of multiplications: $l=2,3$. In the case of $l=2$ there exist two solutions: one well-known solution $\{u=5 ; x=2, y=3, z=4\}$ giving $6^{3}=3^{3}+4^{3}+5^{3}$ and another one giving $709^{3}=193^{3}+461^{3}+631^{3}$ (J.-C. Meyrignac, 2000), while for $l=3$ there exist an infinite number of solutions, and one of them (minimal) gives $422481^{4}=95800^{4}+217519^{4}+414560^{4}$ ELKIES [1988]. 
3) The general polyadic triple $(l \mid p, q)^{(3,2)}$, using (7.4), can be presented in the standard binary form (as (7.1))

$$
\sum_{i=1}^{2 p+1}\left(u_{i}+1\right)^{l+1}=\sum_{j=1}^{2 q+1}\left(v_{i}+1\right)^{l+1}, \quad u_{i}, v_{j} \in \mathbb{Z} .
$$

Let us apply the polyadic Lander-Parkin-Selfridge Conjecture 7.7 for this case: the solutions to (7.14) exist, if $3 \leq l \leq l_{p L S P}=p+q+1$. But the binary Lander-Parkin-Selfridge Conjecture 7.3, applied directly, gives $3 \leq l \leq l_{L S P}=2 p+2 q+1$. So we should have counterexamples to the polyadic Lander-Parkin-Selfridge conjecture, when $l_{p L S P}<l \leq l_{L S P}$. For instance, for $p=q=1$, we have $l_{p L S P}=3$, while the (minimal) counterexample with $l=5$ is $\left\{u_{1}=3, u_{2}=18, u_{3}=21, v_{1}=9, v_{2}=14, v_{3}=22\right\}$ giving $3^{6}+19^{6}+22^{6}=10^{6}+15^{6}+23^{6}$ SUBBA RAO [1934].

As it can be observed from Example 7.8, the arity shape of the polyadic ring $\mathcal{R}_{m, n}^{\mathbb{Z}}$ is crucial in constructing polyadic analogs of the equal sums of like powers conjectures. We can make some general estimations assuming a special (more or less natural) form of its operations over integers.

Definition 7.9. We call $\mathcal{R}_{m, n}^{\mathbb{Z}}$ the standard polyadic ring, if the "leading terms" of its $m$-ary addition and $n$-ary multiplication are

$$
\begin{aligned}
& \nu_{m}[\overbrace{x, x, \ldots, x}^{m}] \sim m x, \\
& \mu_{n}[\overbrace{x, x, \ldots, x}^{n}] \sim x^{n}, \quad x \in \mathbb{Z} .
\end{aligned}
$$

The polyadic ring $\mathcal{R}_{3,2}^{\mathbb{Z}}$ from Example $\mathbf{7 . 8}$, as well as the congruence class polyadic ring $\mathcal{R}_{m, n}^{[a, b]}(6.9)$ are both standard.

Using (7.3), we obtain approximate behavior of the polyadic power in the standard polyadic ring

$$
x^{\langle l\rangle_{n}} \sim x^{l(n-1)+1}, \quad x \in \mathbb{Z}, \quad l \in \mathbb{N}, \quad n \geq 2 .
$$

So increasing the arity of multiplication leads to higher powers, while increasing arity of addition gives more terms in sums. Thus, the estimation for the polyadic analog of the equal sums of like powers Diophantine equation (7.4) becomes

$$
(p(m-1)+1) x^{l(n-1)+1} \sim(q(m-1)+1) x^{l(n-1)+1}, \quad x \in \mathbb{Z} .
$$

Now we can apply the binary Lander-Parkin-Selfridge Conjecture 7.3 in the form: the solutions to (7.18) can exist if $3 \leq l \leq l_{L P S}$, where $l_{L P S}$ is an integer solution of

$$
(n-1) l_{L P S}=(p+q)(m-1)+1 .
$$

On the other hand the polyadic Lander-Parkin-Selfridge Conjecture 7.7 gives: the solutions to (7.18) can exist if $3 \leq l \leq l_{p L P S}=p+q+1$. Note that $(p+q) \geq 2$ now.

An interesting question arises: which arities give the same limit, that is, when $l_{p L P S}=l_{L P S}$ ?

Proposition 7.10. For any fixed number of additions in both sizes of the polyadic analog of the equal sums of like powers Diophantine equation (7.4) $p+q \geq 2$, there exist limiting arities $m_{0}$ and $n_{0}$ (excluding the trivial binary case $m_{0}=n_{0}=2$ ), for which the binary and polyadic Lander-ParkinSelfridge conjectures coincide $l_{p L P S}=l_{L P S}$, such that

$$
\begin{aligned}
m_{0} & =3+p+q+(p+q+1) k, \\
n_{0} & =2+p+q+(p+q) k, \quad k \in \mathbb{N}^{0} .
\end{aligned}
$$


Proof. To equate $l_{L P S}=l_{p L P S}=p+q+1$ we use (7.19) and solve in integers the equation

$$
\left(n_{0}-1\right)(p+q+1)=(p+q)\left(m_{0}-1\right)+1 \text {. }
$$

In the trivial case, $m_{0}=n_{0}=2$, this is an identity, while the other solutions can be found from $n_{0}(p+q+1)=(p+q) m_{0}+2$, which gives (7.20)-(7.21).

Corollary 7.11. In the limiting case $l_{p L P S}=l_{L P S}$ the arity of multiplication always exceeds the arity of addition

$$
m_{0}-n_{0}=k+1, \quad k \in \mathbb{N}^{0},
$$

and they start from $m_{0} \geq 5, n_{0} \geq 4$.

The first allowed arities $m_{0}$ and $n_{0}$ are presented in TABLE 4. Their meaning is the following.

Corollary 7.12. For the polyadic analog of the equal sums of like powers equation over the standard polyadic ring $\mathcal{R}_{m, n}^{\mathbb{Z}}$ (with fixed $p+q \geq 2$ ) the polyadic Lander-Parkin-Selfridge conjecture becomes weaker than the binary one $l_{p L P S} \geq l_{L P S}$, if: 1) the arity of multiplication exceeds its limiting value $n_{0}$ with fixed arity of the addition; 2 ) the arity of addition is lower, than its limiting value $m_{0}$ with the fixed arity of multiplication.

TABLE 4. The limiting arities $m_{0}$ and $n_{0}$, which give $l_{p L P S}=l_{L P S}$ in (7.18).

\begin{tabular}{||c|c||c|c||c|c||}
\hline \hline \multicolumn{2}{||c||}{$p+q=2$} & \multicolumn{2}{c||}{$p+q=3$} & \multicolumn{2}{c||}{$p+q=4$} \\
\hline \hline$m_{0}$ & $n_{0}$ & $m_{0}$ & $n_{0}$ & $m_{0}$ & $n_{0}$ \\
\hline 5 & 4 & 6 & 5 & 7 & 6 \\
8 & 6 & 10 & 8 & 12 & 10 \\
11 & 8 & 14 & 11 & 17 & 14 \\
14 & 10 & 18 & 14 & 22 & 18 \\
\hline \hline
\end{tabular}

Example 7.13. Consider the standard polyadic ring $\mathcal{R}_{m, n}^{\mathbb{Z}}$ and fix the arity of addition $m_{0}=12$, then take in (7.18) the total number of additions $p+q=4$ (the last column in TABLE 4). We observe that the arity of multiplication $n=16$, which exceeds the limiting arity $n_{0}=10$ (corresponding to $m_{0}$ ). Thus, we obtain $l_{p L P S}=5$ and $l_{L P S}=3$ by solving (7.19) in integers, and therefore the polyadic Lander-Parkin-Selfridge conjecture becomes now weaker than the binary one, and we do not obtain counterexamples to it, as in Example $\mathbf{7 . 8}$ (where the situation was opposite $l_{p L P S}=3$ and $l_{L P S}=5$, and they cannot be equal).

A concrete example of the standard polyadic ring (Definition 7.9) is the polyadic ring of the fixed congruence class $\mathcal{R}_{m, n}^{[a, b]}$ considered in SECTION 6, because its operations (6.2) - 6.3) have the same straightforward behavior (7.15)-(7.16). Let us formulate the polyadic analog of the equal sums of like powers Diophantine equation (7.4) over $\mathcal{R}_{m, n}^{[a, b]}$ in terms of operations in $\mathbb{Z}$. Using (6.2)-(6.3) and (7.17) for (7.4) we obtain

$$
\sum_{i=1}^{p(m-1)+1}\left(a+b k_{i}\right)^{l(n-1)+1}=\sum_{j=1}^{q(m-1)+1}\left(a+b k_{j}\right)^{l(n-1)+1}, \quad a, b, k_{i} \in \mathbb{Z} .
$$

It is seen that the leading power behavior of both sides in (7.24) coincides with the general estimation (7.18). But now the arity shape $(m, n)$ is fixed by (6.10)-(6.11) and given in TABLE 3 . Nevertheless, we can consider for (7.24) the polyadic analog of Fermat's last theorem 7.6, the LanderParkin-Selfridge Conjecture 7.3 (solutions exist for $l \leq l_{L P S}$ ) and its polyadic version (Conjecture 7.7. solutions exist for $\left.l \leq l_{p L P S}\right)$, as in the estimations above. Let us consider some examples of solutions to (7.24). 
Example 7.14. Let $[[2]]_{3}$ be the congruence class, which is described by $(4,3)$-ring $\mathcal{R}_{4,3}^{[2,3]}$ (see TABLE 3), and we consider the polyadic Fermat's triple $(l \mid 0,5)^{(4,3)}(7.6)$. Now the powers are $l_{L P S}=8$, $l_{p L P S}=6$, and for instance, if $l=2$, we have solutions, because $l<l_{p L P S}<l_{L P S}$, and one of them is

$$
14^{5}=4 \cdot(-1)^{5}+7 \cdot 5^{5}+8^{5}+2 \cdot 11^{5} .
$$

7.2. Frolov's Theorem and the Tarry-Escott problem. A special set of solutions to the polyadic Lander-Parkin-Selfridge Conjecture 7.7 can be generated, if we put $p=q$ in (7.24), which we call equal-summand solutions 5 , by exploiting the Tarry-Escott problem approach DORWART AND BROWN [1937] and Frolov's Theorem 7.1.

Theorem 7.15. If the set of integers $k_{i} \in \mathbb{Z}$ solves the Tarry-Escott problem

$$
\sum_{i=1}^{p(m-1)+1} k_{i}^{r}=\sum_{j=1}^{p(m-1)+1} k_{j}^{r}, \quad r=1, \ldots, s=l(n-1)+1,
$$

then the polyadic equal sums of like powers equation with equal summands (7.4) has a solution over the polyadic $(m, n)$-ring $\mathcal{R}_{m, n}^{[a, b]}$ having the arity shape given by the following relations:

1) Inequality

$$
l(n-1)+1 \leq p(m-1)
$$

2) Equality

$$
p(m-1)=2^{l(n-1)+1} .
$$

Proof. Using Frolov's Theorem 7.1 applied to (7.26) we state that

$$
\sum_{i=1}^{p(m-1)+1}\left(a+b k_{i}\right)^{r}=\sum_{j=1}^{p(m-1)+1}\left(a+b k_{j}\right)^{r}, \quad r=1, \ldots, s=l(n-1)+1,
$$

for any fixed integers $a, b \in \mathbb{Z}$. This means, that (7.29), with $k_{i}$ (satisfying (7.26) ) corresponds to a solution to the polyadic equal sums of like powers equation (7.4) for any congruence class $[[a]]_{b}$. Nevertheless, the values $a$ and $b$ are fixed by the restrictions on the arity shape and the relations (6.4) and (6.8).

1) It is known that the Tarry-Escott problem can have a solution only when the powers are strongly less than the number of summands BORWEIN [2002], DORWART AND BROWN [1937], that is $(l(n-1)+1)+1 \leq p(m-1)+1$, which gives (7.27).

2) A special kind of solutions, when number of summands is equal to 2 into the number of powers, was found using the Thue-Morse sequence ALLOUCHE AND SHALLIT [1999], which always satisfies the bound (7.27), and in our notation it is (7.28).

In both cases the relations (7.27) and (7.28) should be solved in positive integers and with $m \geq 2$ and $n \geq 2$, which can lead to non-unique solutions.

Let us consider some examples which give solutions to the polyadic equal sums of like powers equation (7.4) with $p=q$ over the polyadic $(m, n)$-ring $\mathcal{R}_{m, n}^{[a, b]}$ of the fixed congruence class $[[a]]_{b}$.

Example 7.16. 1) One of the first ideal (non-symmetric) solutions to the Tarry-Escott problem has 6 summands and 5 powers (A. Golden, 1944)

$$
0^{r}+19^{r}+25^{r}+57^{r}+62^{r}+86^{r}=2^{r}+11^{r}+40^{r}+42^{r}+69^{r}+85^{r}, \quad r=1, \ldots, 5 .
$$

\footnotetext{
${ }^{5}$ The term "symmetric solution" is already taken and widely used BORWEIN [2002].
} 
We compare with (7.26) and obtain

$$
\begin{gathered}
p(m-1)=5, \\
l(n-1)=4 .
\end{gathered}
$$

After ignoring binary arities we get $m=6, p=1$ and $n=3, l=2$. From Theorem 6.8 and TABLE 3 we observe the minimal choice $a=4$ and $b=5$. It follows from Frolov's Theorem 7.1, that all equations in (7.30) have symmetry $k_{i} \rightarrow a+b k_{i}=4+5 k_{i}$. Thus, we obtain the solution of the polyadic equal sums of like powers equation (7.4) for the fixed congruence class $[[4]]_{5}$ in the form

$$
4^{5}+99^{5}+129^{5}+289^{5}+314^{5}+434^{5}=14^{5}+59^{5}+204^{5}+214^{5}+349^{5}+429^{5} .
$$

It is seen from TABLE 3 that the arity shape $(m=6, n=3)$ corresponds, e.g., to the congruence class $[[4]]_{10}$ as well. Using Frolov's theorem, we substitute in (7.30) $k_{i} \rightarrow 4+10 k_{i}$ to obtain the solution in the congruence class $[[4]]_{10}$

$$
4^{5}+194^{5}+254^{5}+574^{5}+624^{5}+864^{5}=24^{5}+114^{5}+404^{5}+424^{5}+694^{5}+854^{5} .
$$

2) To obtain the special kind of solutions to the Tarry-Escott problem we start with the known one with 8 summands and 3 powers (see, e.g., LEHMER [1947])

$$
0^{r}+3^{r}+5^{r}+6^{r}+9^{r}+10^{r}+12^{r}+15^{r}=1^{r}+2^{r}+4^{r}+7^{r}+8^{r}+11^{r}+13^{r}+14^{r}, \quad r=1,2,3 .
$$

So we have the concrete solution to the system (7.26) with the condition (7.28) which now takes the form $8=2^{3}$, and therefore

$$
\begin{gathered}
p(m-1)=7, \\
l(n-1)=2 .
\end{gathered}
$$

Excluding the trivial case containing binary arities, we have $m=8, p=1$ and $n=3, l=1$. It follows from Theorem $\mathbf{6 . 8}$ and TABLE 3, that $a=6$ and $b=7$, and so the polyadic ring is $\mathcal{R}_{8,3}^{[6,7]}$. Using Frolov's Theorem 7.1, we can substitute entries in (7.35) as $k_{i} \rightarrow a+b k_{i}=6+7 k_{i}$ in the equation with highest power $r=3$ (which is relevant to our task) and obtain the solution of (7.4) for $[[6]]_{7}$ as follows

$$
6^{3}+27^{3}+41^{3}+48^{3}+69^{3}+76^{3}+90^{3}+111^{3}=13^{3}+20^{3}+34^{3}+55^{3}+62^{3}+83^{3}+97^{3}+104^{3} .
$$

We conclude that consideration of the Tarry-Escott problem and Frolov's theorem over polyadic rings gives the possibility of obtaining many nontrivial solutions to the polyadic equal sums of like powers equation for fixed congruence classes.

Acknowledgments. The author would like to express his deep gratitude and sincere thankfulness to Joachim Cuntz, Christopher Deninger, Grigorij Kurinnoj, Mike Hewitt, Jim Stasheff, Alexander Voronov, and Wend Werner for discussions, and to Dara Shayda for Mathematica programming help. 


\section{REFERENCES}

Allouche, J.-P. And J. Shallit (1999). The ubiquitous Prouhet-Thue-Morse sequence. In: Sequences and Their Applications. Proceedings of the International Conference, SETA '98, Singapore, December, 1998, London: Springer, pp. 1-16.

BAZunova, N., A. Borowiec, AND R. KERneR (2004). Universal differential calculus on ternary algebras. Lett. Math. Phys. 67 (3), 195-206.

Belousov, V. D. (1972). n-Ary Quasigroups. Kishinev: Shtintsa.

Bergman, C. (2012). Universal Algebra: Fundamentals and Selected Topics. New York: CRC Press.

Boccioni, D. (1965). Caratterizzazione di una classe di anelli generalizzati. Rend. Semin. Mat. Univ. Padova 35, 116-127.

BorweIn, P. (2002). The Prouhet-Tarry-Escott problem. In: P. BORWEIN (Ed.), Computational Excursions in Analysis and Number Theory, CMS books in mathematics: 10, New York: Springer-Verlag, pp. 85-95.

Carlsson, R. (1976). Cohomology of associative triple systems. Proc. Amer. Math. Soc. 60 (1), 1-7.

CARlsson, R. (1980). N-ary algebras. Nagoya Math. J. 78 (1), 45-56.

Celakoski, N. (1977). On (F, G)-rings. God. Zb., Mat. Fak. Univ. Kiril Metodij Skopje 28, 5-15.

Cohn, P. M. (1965). Universal Algebra. New York: Harper \& Row.

Crombez, G. (1972). On (n, m)-rings. Abh. Math. Semin. Univ. Hamb. 37, 180-199.

Crombez, G. And J. Timm (1972). On (n, m)-quotient rings. Abh. Math. Semin. Univ. Hamb. 37, 200-203.

Cuntz, J. (1977). Simple $C^{*}$-algebras generated by isometries. Commun. Math. Phys. 57, 173-185.

Čupona, G. (1965). On [m,n]-rings. Bull. Soc. Math. Phys. Macedoine 16, 5-9.

Denecke, K. And S. L. Wismath (2009). Universal Algebra and Coalgebra. Singapore: World Scientific.

Dorwart, H. L. AND O. E. Brown (1937). The Tarry-Escott problem. Amer. Math. Monthly 44 (10), 613-626.

DupliJ, S. (2012). Polyadic systems, representations and quantum groups. J. Kharkov National Univ., ser. Nuclei, Particles and Fields 1017 (3(55)), 28-59. expanded version arXiv: math. RT/1308.4060v2.

DupliJ, S. (2016). A "q-deformed" generalization of the Hosszú-Gluskin theorem. Filomat (11), 2985-3005.

DuPliJ, S. AND W. MARCINEK (2001). Semisupermanifolds and regularization of categories, modules, algebras and Yang-Baxter equation. Nucl. Phys. Proc. Suppl. 102, 293-297. Int. Conference on Supersymmetry and Quantum Field Theory: D.V. Volkov Memorial Conference, Kharkov, Ukraine, July 2000.

DupliJ, S. AND W. MARCINEK (2002). Regular obstructed categories and topological quantum field theory. J. Math. Phys. 43 (6), 3329-3341.

DupliJ, S. AND W. WERnER (2015). Structure of unital 3-fields, preprint Mathematische Institute, Münster, 17 p., arXiv:math.RA/1505.04393v2.

EKL, R. L. (1998). New results in equal sums of like powers. Math. Comput. 67 (223), 1309-1315.

Elkies, N. D. (1988). On $A^{4}+B^{4}+C^{4}=D^{4}$. Math. Comp 51 (184), 825-835.

Frolov, M. (1889). Égalités à deux degrés. Bull. Soc. Math. Fr. 17, 69-83.

Gal'MAK, A. M. (2003). n-Ary Groups, Part 1. Gomel: Gomel University.

GŁAZEK, K. AND J. Michalski (1984). On polyadic groups which are term-derived from groups. Stud. Sci. Math. Hung. 19, 307-315.

GrëtSER, G. (1968). Universal algebra. New York-Toronto: D. Van Nostrand Co., Inc.

Halmos, P. (1962). Algebraic Logic. New York: Chelsea Publishing.

IANCU, L. AND M. S. Pop (1997). A Post type theorem for $(m, n)$ fields. In: G. HAliC AND G. CRISTESCU (Eds.), Proceedings of the Scientific Communications Meeting of "Aurel Vlaicu” University, Arad, Romania, May 16-17, 1996, Edition III, Volume 14A, Arad: "Aurel Vlaicu” Univ. of Arad Publishing Centre, pp. 1318.

Lander, L., T. Parkin, And J. Selfridge (1967). A survey of equal sums of like powers. Math. Comput. 21, 446-459.

LeEson, J. J. AND A. T. Butson (1980). On the general theory of $(m, n)$ rings. Algebra Univers. 11, $42-76$. 
LeHMer, D. H. (1947). The Tarry-Escott problem. Scripta Math. 13, 37-41.

Lister, W. G. (1971). Ternary rings. Trans. Amer. Math. Soc. 154, 37-55.

MiChAlSKi, J. (1988). On J-derived polyadic groups. Mathematica (Cluj) 30 (53), 149-155.

Mignot, F. (1976). Contrôle dans les inéquations variationelles elliptiques. J. Funct. Anal. 22, 130-185.

Monk, J. D. AND F. SiOSON (1966). $m$-semigroups, semigroups and function representations. Fundam. Math. 59, 233-241.

Nguyen, H. D. (2016). A new proof of the Prouhet-Tarry-Escott problem. Integers 16, A01, 1-9.

Pop, A. AND M. S. Pop (2002). Some embeddings theorems for $(n, 2)$-rings. Bul. Ştiinţ. Univ. Baia Mare, Ser. B, Fasc. Mat.-Inform. 18 (2), 311-316.

RuSAKOv, S. A. (1998). Some Applications of n-ary Group Theory. Minsk: Belaruskaya navuka.

SPIRA, R. (1962). The Diophantine equation $x^{2}+y^{2}+z^{2}=m^{2}$. Am. Math. Mon. 69, 360-365.

SubBa RAO, K. (1934). On sums of sixth powers. J. London Math. Soc. s1-9 (3), 172-173.

WiLES, A. (1995). Modular elliptic curves and fermat's last theorem. Ann. Math. 141 (3), 443-551.

ZupNiK, D. (1967). Polyadic semigroups. Publ. Math. (Debrecen) 14, 273-279.

\section{LIST OF TABLES}

1 "Quantization" of arity $\ell$-shapes 11

2 The arity signature and arity shape of polyadic algebraic structures. 21

3 The polyadic ring $\mathcal{R}_{m, n}^{\mathbb{Z}(a, b)}$ of the fixed residue class $[[a]]_{b}$ : arity shape. 34

4 The limiting arities $m_{0}$ and $n_{0}$, which give $l_{p L P S}=l_{L P S}$ in (7.18). 39

Mathematisches InStitute, UniVersität MÜNSteR

EINSTEINSTR. 62, D-48149 MÜNSTER, DEUTSCHLAND

E-mail address: duplijs@math.uni-muenster.de, sduplij@gmail.com

URL: http://homepages.spa.umn.edu/〜duplij 